Space Sciences Laboratory

University of California

Berkeley, California 94720

Distribution of this document is unlimited.

The Interplanetary Magnetic Field;

Solar Origin and Terrestrial Effects

John M. Wilcox

Technical Report on

ONR Contract Nonr 3656(26)

Project No. NR 021 101)

Partial support from NASA Grants

NsG 243 and NGR 05-003-230

Series No. 9, Issue No. 2

Reproduction in whole or in part is permitted for any purpose of the United States Government. 
Interplanetary Magnetic Field; Solar Origin and Terrestrial Effects

John M. Wilcox

Space Sciences Laboratory

University of California

Berkeley, California

\begin{abstract}
Many observations related to the large-scale structure of the interplanetary magnetic field, its solar origin and terrestrial effects are disby spacecraft

cussed. During the period observed the interplanetary field was dominated by a sector structure corotating with the sun in which the field is predominantly away from the sun (on the average in the Archimedes spiral direction) for several days (as observed near the earth), and then toward the sun for several days, etc. The average sector appears to be a coherent entity with internal structure such that its preceding portion is more active than its following portion. Cosmic rays corotate with the interplanetary field, and there are differential flows associated with the sector pattern. Profound effects on geomagnetic activity and the radiation belts are produced as the sector pattern rotates past the earth. The solar origin of the sector pattern is discussed. The solar source may be associated with the large-scale weak background photospheric fields observed with the solar magnetograph. It is suggested that there may be a rather continual relation between this solar structure and terrestrial responses, of which the recurring M-Region geomagnetic storms are just the most prominent example.
\end{abstract}


The Interplanetary Magnetic Field; Solar Origin and Terrestrial Effects

\author{
John M. Wilcox
}

Observations in the past few years have considerably increased our knowledge of the interplanetary magnetic field and of related processes near the sun and the earth. The theoretical discussion by PARKER (1958) has successfully predicted several properties of the interplanetary medium, including the supersonic flow of the solar wind plasma radially away from the sun, and the stretching of solar magnetic field lines into an Archimedes spiral configuration by the combined effects of the radial plasma flow and the solar rotation. An unexpected feature of the spacecraft observations obtained during the past few years has been that the direction of the interplanetary field usually remains predominantly either away from or toward the sun for several consecutive days. It is the purpose of this review to discuss the observations of the large-scale properties of the interplanetary magnetic field with particular reference to these large-scale polarity patterns, to discuss some geomagnetic effects of the interplanetary field and plasma, and finally to discuss the solar origin of this field and the possible implications of the interplanetary observations for the structure of the sun.

our knowledge of the matters discussed in this review is increasing rapidly as more and more observations are obtained with increasingly refined instruments, and as various portions of the solar cycle are examined. One would expect that some parts of the present review might have to be changed in a similar review written a few years from now. The present effort is intended as a progress report in a rapidly evolving field. Emphasis is on the present state of observational knowledge; therefore some of the earlier 
observations with more primitive instrumentation will not be discussed. I will not hesitate to express personal opinions on some of the observations and to draw inferences whose validity can only be completely determined in the light of future observations. MUSTEL (1967) emphasizes the importance of considering many solar cycles in these investigations. One must always consider the possibility that the observations obtained by spacecraft during a short interval of one solar cycle may be atypical.

An excellent review by DESSLER (1967) has recently discussed several subjects closely related to the present review. The interested reader is referred to Dessler's paper for an interesting historical summary*, a discussion of the conditions necessary for a supersonic flow of plasma from the sun, including the rather surprising result that the flow is not supersonic if both the sun and the corona are too hot, a discussion of extended coronal heating, and of the termination of the solar wind. Dessler's paper could serve as a good introduction to the present considerations. Other basic references are a discussion of theoretical aspects of the solar wind (PARKER, 1963), the Proceedings of the Conference on the Solar Wind held at the California Institute of Technology and the Jet Propulsion Laboratory (MACKIN and NEUGEBAUER, 1966), and the Proceedings of the IAU Symposium on Solar and Stellar Magnetic Fields (LUST, 1965).

*But the contributions of Prof. Hannes Alfvén might be given more prominence. In the early 1930s he was almost alone in emphasizing the importance of the magnetic field in solar and interplanetary considerations. In recent years Alfvén has emphasized the importance of the electric field, a matter which is only recently receiving observational attention. 


\section{THE INTERPLANETARY MAGNETIC FIELD}

By reinterpreting the observations of ionized cometary tails, BIERMANN (1951) came to the conclusion that at all times there exists a flow of plasma moving radially away from the sun. CHAPMAN (1954) pointed out that the large thermal conductivity to be expected in the coronal plasma would mean that this extended coronal plasma near the earth would have a temperature of several hundred thousand degrees. Based on these ideas, PARKER (1958) developed a theory for the hydrodynamic expansion of the corona, and named the resulting streaming coronal plasma the solar wind. The large electrical conductivity of the solar wind plasma means that some of the solar magnetic field lines will be frozen into this streaming plasma and stretched out radially away from the sun. PARKER (1958) pointed out that the combination of a radial soler wind velocity plus the solar rotation will cause the interplanetary magnetic field lines to be stretched out into Archimedes spirals. Near the orbit of the earth the average angle between the interplanetary magnetic field and earth-sun direction would be expected to be about $45^{\circ}$, since the two effects mentioned above are of approximately equal magnitude at I $\mathrm{AU}$, i.e. the product $\Omega \mathrm{R}$ of the solar angular velocity $\Omega$ and a distance $R$ of $1 \mathrm{AU}$ is about $440 \mathrm{~km} / \mathrm{sec}$, and the observed solar wind velocity near $1 \mathrm{AU}$ is often approximately the same value. We may note in passing that the position of the earth is nearly optimum for observing this feature of the interplanetary magnetic field; observations made considerably nearer to the sun would reveal a predominantly radial field, whereas observations made at 2 or 3 AU from the sun would reveal a predominantly azimuthal field, i.e. the Archimedes spiral is tightly wound up at this distance. As the solar wind velocity increases the direction of the interplanetary magnetic field 
becomes more nearly radial, while if the solar wind velocity is small the direction of the interplanetary field becomes more nearly azimuthal.

We can now consider the polarity of the interplanetary field. AHLUWALIA and DESSLER (1962) pointed out that a discussion of the polarity to be expected in the interplanetary field might begin with the observations of the photospheric magnetic field obtained with a solar magnetograph (BABCOCK, 1953). As will be discussed in more detail later, these observations indicated that in the photosphere there are regions of varying sizes in which the solar magnetic field is directed predominantly either out of the sun or into the sun. AHLUWALIA and DESSLER (1962) suggested that such a photospheric region with field directed predominantly out of the sun might be the source of a region of interplanetary field lines with polarity away from the sun, and vice versa. Such a photospheric field region is often observed to recur for several solar rotations, so that it will appear at central meridian approximately every 27 days as observed from the earth. A region of interplanetary magnetic field lines whose origin is in such a photospheric region would then be expected to be observed near the earth every 27 days. It is important to distinguish clearly between the corotation with the sun of such an interplanetary magnetic feature and the possible solid body rotation of outer layers of the solar atmosphere. Near the earth the velocity of the solar wind is nearly radial, and it is only the pattern of the interplanetary field that corotates with the sun. AHLUWALIA and DESSLER (1962) have given an analogy that is useful in understanding this situation. The grooves on a phonograph record corotate with the record, and the needle sliding along the grooves moves nearly radially outward. The spiral interplanetary magnetic field is analogous to the grooves and the plasma sliding along the field lines is analogous to the phonograph needle. Causality is 
reversed in this analogy; in the phonograph record it is the grooves which control the motion of the needle, while in the interplanetary medium the energy density of the streaming solar wind plasma is almost two orders of magnitude larger than the energy density of the interplanetary field, so that it is the radial motion of the plasma that stretches out the interplanetary field lines.

Observations of the Interplanetary Field

Let us compare these theoretical predictions with observations obtained with the IMP-1 satellite in the winter of 1963-64. Figure I shows the distribution of directions of the interplanetary field component parallel to the ecliptic and normal to the ecliptic*. It is clear that on the average the direction of the field is close to that predicted by the Archimedes spiral model. However it is equally clear that this is just an average property of the field. At any given time the field may be found in any direction, including the two quadrants which are not allowed in the simple spiral model. Obviously there exist large amplitude perturbations to the simple spiral model. An impression of the fluctuations of the observed field can be obtained by viewing a movie prepared from observations made by the IMP-I satellite which is available from the author (WILCOX et al., 1966). In spite of these fluctuations it has been found in nearly all the existing observations that if an interval of a few hours is considered the field

*The theoretical difficulties associated with the net southward field component shown in Figure 1 have been discussed by DESSLER (1967). 
direction is clearly recognized as being predominantly either toward the sun or away from the sun.

Figure 1 shows the distributions obtained when the field is averaged over 5.46 minute intervals. The distributions of Figure 2 represent a field averaged over intervals of 3 hours. This distribution is noticeably stretched out near the directions predicted by the spiral model. The 3-hour averaging interval has appreciably decreased the effects of the dynamic fluctuations.

The directions labeled 'positive' in Figure 1 represent an interplanetary field directed predominantly away from the sun; those labeled 'negative' represent an interplanetary field directed predominantly toward the sun. Each 3-hour period of time has been assigned a plus or a minus sign, depending upon the predominant direction of the interplanetary field within that interval. The results are plotted at the periphery of Figure 3, and the inner portion of this figure is a schematic representation of a sector structure that is suggested by these observations. This sector pattern corotates with the sun (consider the analogy with the phonograph record mentioned above). If a sector boundary is observed at the earth at a given time one would expect this same boundary might be observed again about 27 days later. At the time of the storm of 2 December 1963 shown in Figure 3 the solar wind velocity was larger than average, so that the transit time for the boundary to be convected from the sun to the earth was about $11 / 2$ days less than the average transit time. Thus this sector boundary arrived "too soon" as can be seen in Figure 3. It is important to remember that even if the source of the interplanetary sector structure is invariant with time, variations in the solar wind velocity can change the time of arrival of a sector boundary (or of any characteristic feature of the interplanetary field) by 1 or 2 days. 
The regularity of the interplanetary sector pattern will be emphasized in this paper. It will be compared with the considerably less regular patterns of the photospheric magnetic field. Thus it is important that we now examine in some detail just how regular the interplanetary sector description may be. We note in Figure 3 that a sector away from the sun is predominantly populated with plus signs for the 3-hour intervals, and that a sector with field toward the sun is predominantly populated with minus signs. (Occasionally a field direction which is, for example, away may change in a smooth and continuous manner such as to enter a shaded or toward direction in Figure 1 for a few hours, and then return in a smooth manner to the away sectors of Figure 1. This is interpreted as a larger than usual deviation in the direction of a field which still has a polarity directed away from the sun. The later discussion of cosmic rays streaming along interplanetary field lines shown in Figure 21 is consistent with this interpretation.) The validity of the sector description can be judged from Figures 4 and 5 , which show that within a given 3-hour period the field direction is usually confined entirely to either the away or the toward range of directions. The angle $\phi$ in these figures represents azimuthal direction in the ecliptic, and is defined in Figure 1 with $0^{\circ}$ being the direction from the earth to the sun. The ranges of $\phi$ labeled plus and minus in Figure 1 are indicated in Figures 4 and 5. Each point represents \& 5.46 minute interval of time, and it can be seen in Figure 4 that almost all the points lie within the positive (away) range of $\phi$. During most of Figure 5 the field is directed away from the sun, but near the end of the figure its direction abruptly changes to toward the sun. This reversal represents a boundary between two sectors. 
The change of directions occurs between one 5.46 minute point and the next; thus the boundary between the sectors is very narrow. The sectors corotate with the sun and therefore have an azimuthal velocity at the orbit of the earth of about $440 \mathrm{~km} / \mathrm{sec}$. The upper limit of 5.46 minutes for a sector boundary to sweep past the earth represents an upper limit of about 150,000 $\mathrm{km}$ for the thickness of the sector boundary. Examination of observations made with a time resolution of 20 seconds indicates that the thickness may sometimes be considerably less than this.

The validity of the sector description can also be judged from observations obtained with the IMP-2 satellite (FAIRFIELD and NESS, 1967) near the end of 1964. The contours in Figure 6 show the frequency of occurrence of field directions during eight interplanetary sectors observed by IMP-2. Each vertical dashed line indicates the theoretical spiral streaming angle. In each case there is a marked tendency for the field directions to cluster near the theoretical spiral angle and for a paucity of fields in the direction opposite to the spiral angle. The odd numbered sectors in Figure 6 represent field directed toward the sun, and the even numbered sectors represent field directed away from the sun. Fairfield and Ness state that during each sector the ratio of the number of fields along the spiral angle in one direction to that in the opposite direction was typically 100 to 1 . The validity of the sector description during the transition period between the old solar cycle and new solar cycle can be judged from Figure 7, which shows observations obtained with the Mariner 4 spacecraft. The component of the observed field in the direction of the ideal spiral field is plotted for 3-hour intervals. Three successive 27-day Bartels rotations are shown. The sector property of the field is clear in these observations, with only an occasional 3-hour period deviating from it. COLEMAN et al. (1967) concluded that the sector 
polarity patterns are independent of distance from the sun from the earth's orbit to $1.55 \mathrm{AU}$, and over a range of $10^{\circ}$ in the direction normal to the ecliptic.

Figure 3 shows an amusing ${ }^{*}$ example of the reluctance of nature to yield her secrets. The gaps in the 3 hour observations come when the IMP-I satellite is within the region influenced by the geomagnetic field and therefore unable to make interplanetary observations. The orbital period of IMP-I was a few hours less than 4 days, which is approximately the time required for the small toward sector in Figure 1 to rotate past the earth. Each large sector required almost exactly two IMP-1 orbits to rotate past the earth. Furthermore the phase relationship was such that usually when a sector boundary was rotating past the earth IMP-I was within the geomagnetic field and therefore unable to observe it. Thus there was a rather precise coincidence in both period and phase between the rotating sector pattern and the orbital parameters of IMP-I. The possibilities for an artifact in the observations inherent in this situation were examined carefully with negative results. It was none-the-less reassuring to later discover that IMP-2 and Mariner 4 observed a very similar sector pattern at the end of 1964.

*After the IMP-1 sector pattern was later observed also by IMP-2 and Mariner 4. 


\section{Corotation of Interplanetary Field Structures}

We may examine the evidence for the corotation with the sun of the interplanetary field structure. The rotation period of equatorial latitudes on the sun as observed from the earth is about 27 days. If the interplanetary field corotates with the sun it would be expected to show a 27-day recurrence property. An autocorrelation of the direction of the interplanetary field observed with IMP-1 is shown in Figure 8. The 27-day periodicity in the field is obvious. The dashed line in Figure 8 is an autocorrelation of the idealized sector structure sketched in Figure 3; the agreement with the solid line representing the observations again demonstrates the validity of the sector description.

Corotation of field structures can also be examined with simultaneous observations from two spacecraft having an appreciable separation in azimuth. NESS (1966) has examined a time at which IMP-3 and Pioneer 6 were on opposite sides of the earth and separated by $1.3 \times 10^{6} \mathrm{~km}$. Figure 9 shows simultaneous measurements of the interplanetary field vector by the two satellites, with the individual 5.46-minute time averages of IMP-3 advanced by 57.5 minutes to compare remarkably well with the observations by Pioneer 6 . The predicted time lapse for a corotating feature to travel the distance between two satellites is within a few minutes of 57.5 minutes. These observations clearly establish that much of the structure of the interplanetary field is indeed corotating with the sun.

DESSIER (1967, p. 25) has pointed out that the corotation description used above is not unique. The physical situation could also be described in terms of the radial flow of the solar wind through a static spiral interplanetary field of magnitude $V_{S} B_{\perp}$, where $V_{S}$ is the solar wind velocity and $B_{\perp}$ is the magnitude of the normal component of the interplanetary field. As a second alternative to corotation the spiral structure can be visualized 
as being convected by the plasma radially out from the sun. Still, for the quasi-stationary recurring phenomena described in the present review the corotating description is the most convenient. On the other hand, if a solar flare produced a spherically symetric disturbance the corotation description would not be appropriate. For example, in the situation discussed above Pioneer 6 and IMP-3 would detect a spherically symmetric disturbance at the same time, since they are at essentially the same distance from the sun. This is in distinct contrast to the observed delay of 57.5 minutes which arises from their azimuthal separation of $1.3 \times 10^{6} \mathrm{~km}$. The transient phenomena arising from solar flares will not be considered in this review.

Structure within a Typical IMP-1 sector

It appears that a typical sector observed by IMP-1 is a coherent entity in the sense that it has an organized reproducible internal structure. The sector has been defined in terms of the sense (toward or away from the sun) of the interplanetary field. We now examine the magnitude of the interplanetary field as a function of position within an average sector. In Figure 10 the abscissa represents azimuthal position within a sector in terms of the (approximately) 8 days required for one of the large sectors of Figure 3 to rotate past the earth, i.e. day 0 represents the preceding boundary of the sector and day 8 represents the following boundary of the sector. The ordinate represents the average value of the interplanetary field magnitude at each position within the sector. Results are shown separately for away sectors (plus), toward sectors (minus) and all sectors combined (open circles). The average magnitude rises to a peak of greater than $6 \gamma\left(1 \gamma=10^{-5}\right.$ gauss) early in the sector and declines to about $4 \gamma$ in the trailing portion of 
the sector. The fluctuations about this trend observed in Figure 10 are partially caused by statistical fluctuations in the limited sample. Figure 11 shows a similar analysis of the solar wind velocity observed by the MIT plasma detector on IMP-1. The average velocity also reaches a maximum in the early portion of the sector and then has a more or less monotonic decline in the trailing portion of the sector. Thus the field magnitude and solar wind velocity have roughly the same sort of variation within a sector. The density distribution within a typical sector is considerably different, as shown in Figure 12. After reaching a maximum in the early portion of the sector, there is a precipitious decline in the density to less than half of the maximum value in the central portion of the sector, and then an increase in density in the trailing portions of the sector. Thus the typical IMP-1 sector has an internal structure which suggests that it should be considered as an organized entity. The quiet solar wind does not boil at random from the sun but rather is very much structured and guided. In future sections we will examine the internal structure of a sector with regard to cosmic rays and geomagnetic activity.

\section{Evolution of Sectors}

The sector pattern in the winter of 1963-4 has now been described in some detail. A broader perspective can be gained by examining the evolution of the sector pattern during the several years that it has been observed by spacecraft. Figure 13 shows the daily geomagnetic character index $C 9$ as prepared by the Geophysical Institute in Gobttingen. Geomagnetic activity for each day is characterized on a scale running from 0 to 9 , and the physical size of the numbers increases with their magnitude. The index is plotted 
using Bartels solar rotation periods of 27 days. Thus a recurrent structure having a period of about 27 days will appear as a vertical column while, for example, a structure having a period of 28 days will slant downward toward the right. The polarity of the interplanetary field observed by spacecraft is indicated by a light shading for away sectors and a dark shading for toward sectors. During most of 1964 the interplanetary field was not observed by spacecraft, but an interpolation of the sector pattern has been made as indicated in Figure 13. This quiet period at the end of the last solar cycle is of particular interest in the study of solar-terrestrial physics because the large-scale sector pattern appears to have been quasi-stationary. With significant variations with time removed, other features of the interaction between the sun and the earth can perhaps be more readily perceived. The evidence for the sector interpolation during 1964 will be mentioned briefly here and in more detail in later sections of this review as appropriate. The sector pattern observed by IMP-1 at the start of 1964 and by IMP-2 and Mariner 4 at the end of 1964 are very similar, as can be seen in Figure 13. The response of geomagnetic activity as a function of position in an average sector was very similar for IMP-1 and for IMP-2, suggesting that the structure within an average sector did not change appreciably during 1964. Recurring geomagnetic activity is larger in the preceding portion of sectors, so that the recurring geomagnetic activity observed during 1964 can be used to approximately define the boundary of the sector. It is important to note that the precise position of any interpolated boundary during 1964 is uncertain by at best 1 or 2 days. Additional evidence for the interpolation is that cosmic ray observations with the Vela 3 satellite (ASBRIDGE et al., 1967) in the middle of 1964 indicated that the sector pattern at that time was approximately the same as the IMP-I sector pattern. 
It can be seen in Figure 13 that the sector pattern has been a prominent feature of most of the spacecraft observations obtained so far. Whether the solar activity associated with the coming maximum in the sunspot cycle will seriously disrupt the sector property remains to be seen. It is my guess that it will not. Up to about the end of 1964 the interplanetary sector pattern appears to be associated with the old solar cycle. The pattern has an approximately 27-day recurrence time, as can be seen from the vertical pattern of the sector structure when plotted on a 27-day calendar (see also the autocorrelation of the IMP-1 field direction in Figure 8). From the latter part of 1963 to the end of 1964 the sector pattern was changing very little with time, if the interpolation described above is correct. The change from the old solar cycle to the new cycle appears to occur at the end of 1964 and the beginning of 1965 . If a particular Bartels rotation were to be selected it might be number 1799, in which a large toward sector that had probably existed for well over a year in essentially the same form was changed in this rotation to an away polarity. Two changes appear in 1965 as compared with 1964: the evolution with time of the sector pattern becomes more prominent and the recurring sectors slant downward to the right in Figure 13, consistent with a 28-day recurrence period. The 28 -day recurrence period can also be observed in Figure 14, which is an autocorrelation of the interplanetary field direction observed by IMP-3 in 1965. The recurrence peak early in the new cycle is at 28 days, as compared with the 27-day recurrence shown late in the old cycle in Figure 8. This change in recurrence interval is consistent with the observation by BARTELS (1963) that the recurrence interval in geomagnetic activity in the two years before sunspot minimum is 27.0 days, and in the two years after sunspot minimum is 27.75 days. The increase in the sector recurrence interval to 28 days is probably associated with the high latitude

*This change is shown in more detail in Figure 7. 
activity of the new sunspot cycle. Near the end of the old cycle there is little new activity to break up the large-scale magnetic patterns in the photosphere, so they can persist in a quasi-stationary manner over wide intervals of heliographic latitude and longitude. As activity increases in the new cycle these patterns are interrupted by new activity and the resulting interplanetary sector pattern shows a more rapid evolution.

\section{Solar Wind Plasma}

The solar wind velocity and density have a systematic variation within an average IMP-1 sector, as was shown in Figures 11 and 12 . Figure 15 shows the variation of plasma velocity, proton density, and magnetic field strength during 3 Bartels 27-day rotations observed by Mariner 2. Several recurrent peaks in solar wind velocity can be seen. There was a tendency for the field to be unusually strong on the leading edge of the high velocity plasma streams, in agreement with the IMP-1 result shown in Figure 10 . In a frame moving with the average solar wind velocity the ratio of the magnetic energy density to the plasma thermal energy density is of interest. The frequency of occurrence of this ratio for the Mariner 2 observations is shown in Figure 16. This histogram is centered on a ratio equal to 1 , with a ratio between 9/16 and 16/9 for 70\% of the 3-hour intervals. The Mariner 2 frequency distribution of the 3-hour average values of the Alfven velocity is shown in Figure 17. The Alfvén velocity was within the range 30 to $100 \mathrm{~km} / \mathrm{sec}$ for $82 \%$ of all the 3-hour intervals considered, while the average solar wind velocity during this time was approximately $500 \mathrm{~km} / \mathrm{sec}$ (NEUGEBAUER and SNYDER, 1966). The 3-hour average solar wind velocity was always at least twice as great as either the 3-hour average Alfvén velocity or the 3-hour average of 
the fast-wave velocity. Thus the flow of the solar wind was always supersonic for hydromagnetic waves. Two similarities between the Mariner 2 and IMP-I observations have been pointed out by COLEMAN et al. (1966). Each of the 4 field reversals (sector boundaries) observed by Mariner 2 occurred at the time of local minimum in the solar wind velocity. Further, each Mariner 2 reversal was followed within 2.5 days by a local maximum in solar wind velocity. The same features for the IMP-1 observations can be seen in Figure 11. Mariner 2 observed several fairly well established high velocity streams during each solar rotation period, while there were only two polarity reversals during each period. It seems possible that each recurrent high velocity stream observed by Mariner 2 corresponds to some extent to a sector observed by IMP-1. The cosmic ray observations to be discussed in a later section are of interest in this regard.

A comparison by HUNDHAUSEN et al. (1967b) of plasma measurements on the Vela 3 satellite and magnetic field measurements on the IMP-3 satellite has revealed the existence of an anisotropic proton temperature distribution in which the direction of maximum temperature was aligned with that of the magnetic field lines. The detailed temporal behavior of the anistropy and field directions followed the same pattern during periods of slow changes and also during the passage of a "magnetic filament" past both satellites. Figure 18 shows a contour mapping of a typical proton velocity distribution function derived from Vela 3 data. The abscissa is the radial direction from the sun and the ordinate is normal to the radial direction and in a plane tipped by about $55^{\circ}$ to the ecliptic. The contours are drawn at $1 / 10,2 / 10 \ldots$ of the maximum value. Also shown in the figure is the projection in the plane of measurement of the interplanetary magnetic field observed by the nearby IMP-3 satellite. 
In Figure 18 the direction of maximum temperature (defined as being proportional to the mean square random velocity) is parallel to the direction of the magnetic field lines and is directed away from the sun. (This observation took place in a toward sector so the magnetic field direction is toward the sun.) The maximum temperature corresponding to Figure 18 is $9.2 \times 10^{4}{ }^{\circ} \mathrm{K}$, and the ratio of the maximum temperature to the average temperature normal to the direction of maximum is 3.4. This is slightly higher than the average value of this ratio obtained during the period studied. In Figure 19 the magnetic field direction and the direction of maximum proton temperature are compared during a 6-hour period, and it can be seen that the two directions are nearly the same. Figure 20 shows a scatter plot of simultaneous values of the magnetic field direction and the direction of maximum proton temperature obtained during the period July 24 to August 26, 1965. The points that cluster about the line corresponding to zero difference between the two directions are in away sectors, and the points that cluster about the lines corresponding to $180^{\circ}$ difference in the two directions are points in toward sectors. These asymmetric temperature distributions imply a transport of energy away from the sun along the magnetic lines of force with a typical value of $10^{-5}$ ergs $\mathrm{cm}^{-2} \mathrm{sec}^{-1}$. A similar energy transport by electron heat conduction would be expected to be several orders of magnitude larger. A thermal anisotropy in the solar wind plasma has also been observed by WOLFE et al. (1966). NEUGEBAUER and SNYDER (1967) have questioned the temperature anisotropy on the basis of their Mariner 2 observations, but the results discussed above appear to be quite convincing.

The directions of the solar wind velocity determined from Vela 2 observations show that the flow deviates from a simple radial pattern by a perhaps surprising amount (HUNI)HAUSFN et al., 1967a). For periods of at least a 
hour the direction of the flow may deviate by as much as $10^{\circ}$ from the radial. The average direction of flow during the year from July 1964 to July 1965 is from about $11 / 2^{\circ}$ east of the sun, which corresponds to a mean azimuthal velocity component in the ecliptic plane of about $10 \mathrm{~km} / \mathrm{sec}$. A similar velocity has been obtained by BRANDT (1967) from observations of the aberration of ionized cometary tails. These interesting results will be discussed in a later section on the sun.

\section{Interplanetary Filaments}

Considerable evidence has accumulated to indicate that within a sector that takes several days to rotate past the earth, there can exist a smaller ordered structure that may take a time of the order of an hour to rotate past the earth. An example is the feature shown in Figure 9 at 0325 UT, where the field direction changed abruptly by about $180^{\circ}$ and then returned to its original direction in the subsequent half hour. This feature was observed by both Pioneer 6 and IMP-3 with a time delay appropriate to a corotating feature.

MC CRACKEN and NESS (1966) have shown that the solar-generated cosmic radiation is often markedly anisotropic, and that the direction of the maximum cosmic ray flux is parallel to the interplanetary magnetic field. Figure 21 shows the magnetic field and cosmic ray azimuth averaged over successive 7.5minute intervals and projected onto the ecliptic plane. The amplitude of the cosmic ray anisotropy is indicated by the length of the cosmic ray anisotropy arrows. It is evident that despite major changes in the interplanetary field direction, the cosmic ray anisotropy remained well aligned with the field. When a filament boundary rotates past the spacecraft the cosmic ray anisotropy can change appreciably in a time corresponding to the spatial displacement 
of approximately one cyclotron radius of a $13 \mathrm{MeV}$ proton (the mean energy of the cosmic ray detector channel under discussion). (In the observed field directions at the periphery of Figure 3 the plus signs for a few 3-hour intervals are surrounded by parentheses to indicate times when the field changed in a smooth and continuous manner beyond the normally allowed limits of an away sector. The most kinked portion of Figure 21 presumably corresponds to a similar situation.)

Another example of interplanetary filaments observed by BARTLEY et al. (1966) is shown in Figure 22, which shows the counting rate in a detector looking toward the sun and in a detector looking anti-sun. Several examples of the abrupt appearance of a large anisotropy can be observed. Bartley et al. proposed that the cosmic rays are being constrained to move through the interplanetary medium along well-defined filaments of the interplanetary magnetic field. They observed that the spacecraft was within any single filament for a period ranging from 0.4 to 4 hours in duration, corresponding to filamentary diameters ranging between 0.5 and $4 \times 10^{6} \mathrm{~km}$. Bartley et al. observed a pronounced anisotropy for at least 48 hours during the period December 30, 1965 to January 1, 1966. They attribute this to a prolonged storage of the cosmic rays in the immediate vicinity of the sun, with the time constant of the storage being comparable with or greater than 48 hours. An example of the effect of a magnetic filament on the solar wind temperature anisotropy direction is shown in Figure 19, beginning at about 1600 UT. The direction of the temperature anisotropy changed abruptly so as to maintain its alignment with the interplanetary field direction. During the passage of this particular filament past the Vela $3 a$ satellite, none of the parameters usually employed to describe the state of the solar wind ion component showed a significant change, i.e. the density, mean velocity, 
temperature or $\mathrm{He}^{++} / \mathrm{H}^{+}$density ratio. However in other filaments observed by the Vela satellites (STRONG et al., 1967) these parameters were slowly changing inside the filaments, with relatively fast changes at the boundaries between the filaments. The observed spacings of the filament boundaries suggests that the widths of the filaments are of the order of $2 \times 10^{6} \mathrm{~km}$. Changes in solar wind velocity and temperature associated with several filaments observed by Vela 2 are shown in Figure 23. The step-wise changes in Figure 23 are associated by HUNDHAUSEN et al. (1967c) with the filamentary structure of the plasma. GOSLING et al. (1967) have observed several events in which entirely different plasma environments can exist in the solar wind with a small separation and without a shock structure between the two regimes. They interpret such structures as perhaps an extension of coronal streamers out to 1 AU.

\section{Recurrent Proton Streams}

The away sector shown in Figure 3 between 4 December and 12 December is associated with a recurrent series of geomagnetic disturbances which appeared during more than 20 consecutive solar rotations (see discussion in a later section). During most of these recurrences, the sector was populated with a stream of protons having an energy of a few MeV. Figure 24 shows the counting rate of the University of Chicago experiment on IMP-I detecting protons having an energy of a few MeV (FAN et al., 1966). The boundaries of the away sector mentioned above are superimposed on the figure. The recurring stream of protons is for the most part contained within this away sector. The proton counting rate as a function of position within the sector is somewhat similar to the solar wind velocity and interplanetary field magnitude as a function of position within sectors, as was shown in Figures 10 and 11 . FAN et al. 
(1966) have discovered 3 more recurrences of this proton stream in the 3 solar rotations following those shown in Figure 24. BRYANT et al. (1965) have observed 7 recurrences of this proton stream with the Goddard cosmic ray experiment on Explorer 14 in the period February to July, 1963, as shown in Figure 25. The vertical dashed line in Figure 24 is the same 27-day fiducial marker shown in Figure 25. Thus the recurring proton stream was observed to exist from at least February 1963 to April 1964. These recurrence events are characterized by both a very low intensity level (generally several orders of magnitude below that of a moderate-size solar proton event at these energies) and a very steep energy spectrum. In contrast to the solar proton event, no velocity dispersion is observed among the various energy groups. Thus when a stream is encountered it is observed that, for example, both 3 and $10 \mathrm{MeV}$ particles are present simultaneously, indicating that a quasi-equilibrium state has been established (BRYANT et al., 1965). The integral energy spectra observed by Bryant et al. in 1965 for several of these recurrent proton streams are shown in Figure 26. In general, the spectrum did not vary markedly during the course of an event. The differential energy spectrum observed by FAN et al. (1966) during the December 1963 recurrence of the proton event is shown in Figure 27. The spectrum in the leading region of the corotating field seems to be flatter than in the trailing region. With the aid of further observations with the OGO-l satellite FAN et al. (1966) established that helium nuclei are associated with the proton spectrum in these corotating regions.

Perhaps the first question to be discussed with regard to the origin of these protons is whether the acceleration occurs near the surface of the sun or in interplanetary space, perhaps at the turbulent interface between a fastermoving plasma and a slower-moving plasma. BRYANT et al. (1965) prefer the latter 
interpretation as a working hypothesis. FAN et al. (1966) suggest that acceleration near the sun is supported by the fact that not all sectors contain enhanced fluxes of protons or helium nuclei, and that the observed persistence of the spatial structure of the proton intensity raises the possibility of protons streaming from a region where the magnetic "channel" connects with the sun. Further evidence can be cited against acceleration in interplanetary space. During the first recurrence shown in Figure 24 the solar wind velocity measured by the MIT detector was at a maximum at the time of the preceding sector boundary, but no recurring protons were observed at this time. Also, recurrent protons continued to be observed in the trailing portions of the sector when the solar wind velocity was decreasing, such that higher-velocity regions would not be overtaking slower-velocity regions. The similarity of the structure of the recurring protons within the sector to the structure of the interplanetary field magnitude and solar wind velocity (i.e. a rapid rise in the preceding portion of the sector and a more gradual decline in the trailing portion of the sector) appears to be evidence for the acceleration of the recurring protons in a solar region related to the source of the sector structure.

One might wonder whether the other sectors shown in Figure 3 might also have been populated with recurring proton streams with a lower intensity than in the stream discussed above. FAN et al. (1966) in studies with the OGO-1 satellite found a proton and helium component persisting at a low intensity level at all times late in 1964. These fluxes would have appeared as only a small background in the IMP-1 counting rate. It is clear from Figures 24 and 25 that the maximum intensity in the recurring proton stream varies considerably from one recurrence to the next. Since the intensity often increases 
from one recurrence to the next there must be a continual acceleration process. The protons might be accelerated by flares and stored in the sector structure near the sun, or they might be accelerated by an unknown mechanism related to details of the sector structure at the sun. The sector in Figure 3 from 4 December to 12 December clearly was populated with a recurring proton stream at a much larger intensity than existed in any other sector. Why this should be is not understood. It might be related to acceleration processes, storage processes, or the possibility for energetic particles to escape from the sun into interplanetary space.

O'GALLAGHER and SIMPSON (1966) observed several recurring proton streams using simultaneous observations by earth satellites and the Mariner 4 space probe. All these effects observed during 1965 were of very small magnitude as compared with IMP-1 results in 1964. The nature of the recurring streams observed in 1965 appears to be similar to that discussed above for the earlier observations.

Figure 24 shows that the long-lived recurrent proton stream was almost completely contained within one away sector of Figure 3. This observation suggests that most of the interplanetary field lines in this away sector do not join smoothly to interplanetary field lines that return to the sun in the adjacent toward sectors. If there were a smooth connection between the away sector and the toward sectors the recurrent protons would have sufficient time to follow the field lines and thus should appear also in the adjacent toward sectors. Thus energetic particles can be used as "tracers" to yield knowledge of some properties of the interplanetary field, as was also the case in the comparison of solar cosmic ray anisotropies with field directions in Figure 21. 
Cosmic Ray Effects

The counting rate of the Deep River neutron monitor as a function of position within an average sector observed by IMP-1 is shown in Figure 28. The counting rate tends to increase throughout most of the sector, this being consistent with the viewpoint that galactic cosmic rays are excluded by kinks and irregularities in the interplanetary magnetic field. Early in the sector where the interplanetary field magnitude is largest the cosmic ray intensity is smallest, and vice versa. Most of the increase shown in the positive sectors in Figure 28 occurs in only one of the two positive sectors.

The diurnal variation of cosmic ray intensity during the period August 27 , 1962 to December 25, 1963 has been analyzed by MORI et al. (1964), using the meson monitor at Tokyo. Figure 29 shows the average differential anisotropy obtained for each day of the Bartels 27-day solar rotations during this period, i.e. the average diurnal variation over the entire period is subtracted from the average diurnal variation for each Bartels day. The additional anisotropic flows of cosmic rays are indicated in Figure 29. MORI et al. (1964) have thus observed a sector structure that is very similar to the IMP-1 magnetic sector structure shown in Figure 3. The boundaries agree to within about one day. In Figure 29 the sectors from day 6 to 14 and from day 22 to 27 correspond to the IMP-I sectors with field directed away from the sun. The differential outward flow of cosmic rays in these two sectors sketched by MORI et al. (1964) in Figure 29 may be related to the observation that the average magnitude of the interplanetary field was usually slightly larger in away sectors than in toward sectors, as can be seen in Figure 10. Thus these sectors may have been slightly more effective in excluding cosmic rays from the region inside the orbit of the earth. 
RYDER and HATTON (1967) have studied the diurnal cosmic ray variation using a wide band filter applied to the pressure-corrected intensity recorded by the Deep River neutron monitor during the period observed by IMP-I. They analyzed the toward sectors shown in Figure 3 from 12 December to 20 December and the away sectors from 20 December to 1 December. Figure 30 shows the results obtained by subtracting the mean diurnal variation during the central 4 days of the sector from the diurnal variation of days 1 and 2 in the sector (b), from days 3 and 4 in the sector (c), from days 5 and 6 in the sector (d), and from days 7 and 8 in the trailing portion of the sector (e). The observed diurnal variation is essentially constant during the central interval of the sector. However there are additional waves having maxima between $0600-1200$ hours local time in the preceding portion of the sector and between $1800-2400$ hours local time in the trailing portion of the sector. Under the assumption that the exponent of the variational spectrum is equal to zero on the average throughout a sector, Ryder and Hatton derive the differential streaming pattern within a sector shown in Figure 31. A radial streaming exists such that there is a net motion of cosmic ray particles away from the sun near the leading edge of a sector and towards it near the trailing edge, with a velocity of about $200 \mathrm{~km} / \mathrm{sec}$ parallel to the interplanetary field lines. This result appears to be physically plausible since Figures 10 and 11 show that both the solar wind velocity and field magnitude are above average in the preceding portion of the sector and below average in the trailing portion. Galactic cosmic rays might be excluded more efficiently than average in the preceding portion and less efficiently than average in the trailing portion of the sector.

MC CRACKEN et al. (1966) using an elegant cosmic ray anisotropy detector on Pioneer 6 have observed a number of recurrent Forbush decreases similar to the decrease in the positive sector shown in Figure 28. RAO et al. (1967) 
point out that a sector which produces a large Forbush decrease in cosmic ray intensity also tends to produce recurring geomagnetic storms and to be populated with recurrent proton streams. This is consistent with the observations on IMP-I of the away sector from 4 December to 12 December shown in Figure 3. Mc Cracken and coworkers conclude that each recurrent series is the result of a shock wave initiated by a continuous emission of fast plasma by restricted areas of the sun. Some arguments against the shock wave hypothesis have been given in the above section on recurring proton streams.

TERRESTRIAL EFFECTS OF THE INTERPLANETARY SECTOR STRUCTURE

There are several changes in geomagnetic activity and the radiation belts as the interplanetary sector structure rotates past the earth. We may examine some physical reasons for this. The interplanetary magnetic field lines are "draped" through the magnetosheath onto the geomagnetic lines at the magnetopause, according to the observations of FAIRFIELD and NESS (1967). Figure 32 shows the average direction of the interplanetary field and of the magnetosheath field for each of the IMP-2 sectors. It is clear that the average direction of the magnetosheath field is approximately $180^{\circ}$ different for away sectors as compared with toward sectors. We may also note that low energy electrons ( $>40 \mathrm{kev}$ ) and protons (approximately $0.5 \mathrm{MeV}$ ) have full and essentially immediate access from interplanetary space to some geomagnetic field lines (IIN and ANDERSON, 1966; KRIMIGIS et al., 1967). Krimigis et al. found that the proton intensities after a solar flare observed by the satellite Explorer 33 outside the magnetosphere and by the satellite Injun 4 over the earth's polar caps were very similar, as is shown in Figure 33. They interpret their results in terms of a merging between geomagnetic and interplanetary fields, but point out that an anamolous diffusion of the plasma across the magnetic field could possibly be involved. 
An analysis of the geomagnetic activity index 24 -hour sum $k_{p}$ as a function of position within the large sectors of Figure 3 is shown in Figure 34 . The variation in $\mathrm{K}_{\mathrm{p}}$ is considerable, ranging from a maximum greater than 25 to a minimum less than 10. Geomagnetic activity reaches a maximum at about the second day of the sector, and then has a more or less monotonic decline somewhat similar to the variation of field magnitude and solar wind velocity shown in Figures 10 and 11 . The variation of $K_{p}$ as a function of position within away sectors is remarkably similar to the variation within toward sectors, as can be seen in Figure 34. Figure 35 shows that an approximation to the average behavior shown in Figure 34 can be seen for each individual sector observed by IMP-1. The sector boundaries are overlaid on a Bartels musical-diagram chart of geomagnetic activity.

The daily changes in intensity and spatial extent of the radiation zone electrons with energies approximately $1.5 \mathrm{MeV}$ have been studied by ROTHWELI and GREENE (1965). Figure 36 shows intensity contours of these electrons on an $I$ versus time map, where $\mathrm{L}$ is approximately the distance in earth radii at which a particular geomagnetic field line intercepts the geomagnetic equatorial plane. The interplanetary sector structure observed by Mariner 4 is indicated at the top of Figure 36. The boundary of the stable trapping zone was moving outward around the time of each sector boundary, and reached its maximum distance from the earth about a day and a half after the boundary. The spatial extent and intensity of energetic electrons in the radiation belts increased after every sector boundary. Rothwell and Green suggest that injection of fresh particles into the magnetosphere probably occurs near the sector boundaries.

WILLIAMS (1966) finds that throughout the outer radiation zone ( $\mathrm{L} \geqslant 3.5)$ the trapped energetic electron population exhibits a clear 27-day periodicity. 
The arrival of sector boundaries in which the field changes from toward the sun to away from the sun trigger the major electron intensity increases, but smaller changes in the electron intensities can be seen near the arrival of the other boundaries. An example is shown in Figure 37.

The occurrence frequency of the short-period geomagnetic fluctuations known as pearl pulsations as a function of position within a sector has been studied by MC PHERRON and WARD (1967). Figure 38 shows the probability of pearl occurrence as a function of position within a sector and of local time. The enhanced probability of occurrence of pearl pulsations on the days of the sector crossings is evident. Pearls are likely to occur at almost any hour near the day of a sector crossing. In the middle of a sector, however, they are limited to times between local noon and sunset.

NISHIDA (1966) has studied the ring current intensity, represented roughly by the daily mean horizontal intensity at Honolulu, during the period observed by IMP-1. Figure 39 shows the result for 3 Bartels solar rotations. Arrows indicate the passage of a sector boundary. The maximum depression in the horizontal intensity seems to appear about a day after the passage of a sector boundary.

A stability in the response of a geomagnetic activity index (24-hour sum $\mathrm{K}_{\mathrm{p}}$ ) as a function of position within a sector over an interval of a year is shown in Figure 40, which compares observations made with IMP-1 at the start of 1964 and with IMP-2 near the end of 1964. The average value of $K_{p}$ during the period observed by IMP-2 was somewhat less than for IMP-1 because of the decline in solar activity through the year 1964. The shape of the two curves is very similar, including even the knee or point of inflection in the middle of the sector. 
During the period in 1965 observed by IMP-3, toward sectors were predominant, but the small young away sectors produced the largest geomagnetic activity. Figure 41 shows that for each Bartels rotation in this period the away sectors produced a larger average value of $K_{p}$ than did the toward sectors.

The above observations indicate that profound systematic changes occurred in geomagnetic activity and in the radiation belts as the interplanetary sector structure rotated past the earth.

\section{The Axial-Equinoctial Controversy}

Geomagnetic activity is observed to have semiannual peaks located near (BARTELS, 1963) the equinoxest. These are also times when the heliographic latitude of the earth reaches its maximum excursion of $7^{\circ}$ away from the solar equator. For many years a lively controversy has existed as to whether these semiannual maxima occur because at this time a radius vector from the earth to the sun CORTIE, 1913
would come nearest to the zones of solar activity (the axial hypothesis,y, or whether the maxima are caused by the fact that at the equinoxes the geomagnetic field is most nearly normal to the direction of the incident solar wind plasma (the equinoctial hypothesiş). We do not propose to review these many years of discussion, but rather merely to cite some recent evidence in favor of the equinoctial hypothesis.

The semiannual maxima in geomagnetic activity can be clearly seen in the Bartels $K_{p}$ planetary indices of geomagnetic activity. If these maxima are associated with the orientation of the geomagnetic dipole axis with regard to the radial flow of the solar wind, then one might expect also a diurnal variation in geomagnetic activity caused by the $12^{\circ}$ tilt of the geomagnetic axis with respect to the rotational axis of the earth. The $K_{p}$ index shows essentially no diurnal variation (MICHEL, 1964), because Bartels combined the 
observations of the various geomagnetic observatories in a manner such as to remove any diurnal variation. ${ }^{*}$ MAYAUD (1967) has recently proposed some new indices of geomagnetic activity to supplement the $\mathrm{K}_{\mathrm{p}}$ indices. These are prepared separately for the northern hemisphere $\left(K_{n}\right)$ and for the southern hemisphere $\left(K_{S}\right)$. A careful effort is made to use observatories whose distribution in longitude is homogenous. Following MC INTOSH (1959), a first cause for a universal time daily variation is assumed to be the daily variation of the dipole axis orientation. We assume that the activity is larger when the angle between the dipole axis in the sun-earth direction is close to $90^{\circ}$. The variation of this angle is out of phase at the two solstices (for instance it is closest to $90^{\circ}$ at $0430 \mathrm{UT}$ and farthest at $1630 \mathrm{UT}$ ); at the equinoxes, it is equal to $90^{\circ}$ at 1030 UT and at 2230 UT. Figure 42 shows the daily variation curves of linear indices related to $K_{n}$ and $K_{S}$ for the differences June minus December (solstices), and for the equinoxes minus the average of June and December. In the first case maximum and minimum occur just at the predicted hours: 0430 UT and 1630 UT. In the second case a prominent maximum occurs at the predicted time of $2230 \mathrm{UT}$, a much smaller maximum is somewhat displaced from the other predicted time of 1030 UT. Thus the predictions of the equinoctial explanation are to a considerable extent fulfilled by the diurnal variation of Mayaud's indices of geomagnetic activity.

If the orientation of the geomagnetic lines with respect to the solar wind is important for producing geomagnetic activity, one might expect that

* Since BARTELS (1932) believed in the equinoctial explanation, this was perhaps a bit inconsistent. 
at the June solstice when the northern geomagnetic axis is tipped toward the solar wind that geomagnetic activity in the north should tend to be larger than in the south, and vice versa for the December solstice. This effect is clearly seen in Figure 43, which shows the annual variation of linear indices related to Mayaud's $K_{n}$ and $K_{s}$. In June, for example, the northern hemisphere is the more active while in December the southern hemisphere is the more active. Mayaud's new indices appear to provide a valuable supplement to Bartels $\mathrm{K}_{\mathrm{p}}$ indices.

Another possibility for comparing the axial and equinoctial theories arises from the fact that the sun's axis is most inclined toward the earth on September 7 and March 5, while equinoxes occur on September 23 and March 20, so that there are differences of about 16 days between the two events. If the axial theory is invoked, a 5 day transit time for the solar wind from the sun to the earth might be included, which would then reduce this difference to 11 days. BARTELS (1932, 1963) has made several investigations to attempt to determine the precise time of the semiannual maxima in the amplitude of geomagnetic activity. His colleague, MEYER (1966), has investigated the precise time of the semiannual maxima in the recurrence tendency of geomagnetic activity using the harmonic dial method, with results shown in Figure 44 . The results are consistent with the equinoctial hypothesis, and the probable errors shown by the circles do not favor the axial hypothesis.

The time displacement between the predictions of the axial and the equinoctial theories has also been investigated by ROOSEN (1966). Figure 45 shows the semiannual variation of the average $A_{p}$, a linear measure of geomagnetic activity. The full curve shows the absolute solar declination (equinoctial hypothesis) and the broken curve shows the absolute heliographic latitude of the earth (axial hypothesis). The form of the variation of 
geomagnetic activity is very similar to the form of the curve for the equinoctial hypothesis in that it has sharp maxima at the equinoxes and smooth minima at the solstices. Roosen has derived a method for correcting a $K_{p}$ observed at a given date to the equivalent equinoctial $K_{p}$. The results are shown in Figure 46.

The above evidence suggests that if the axial hypothesis is to be invoked a phase lag in the response of geomagnetic activity of about 15 days must be explained. PRIESTER and CATTANI (1962) suggested that a semiannual density variation of charged solar particles which are trapped in the earth's magnetosphere could provide the required phase lag mechanism. Such a phase lag seems unlikely because the analysis of ROTHWELI (1965) shown in Figure 36 shows a large scale change in the radiation belts following within a few days of the passage of a sector boundary. The analysis of WIICOX et al. (1967) shows that the peak in the crosscorrelation as a function of time lag between $K_{p}$ and the interplanetary field magnitude occurs at a lag of zero days, as shown in Figure 47.

Several authors (see for example SAITO, 1965) have suggested that some recurring geomagnetic disturbances seem to show an annual variation such that a particular sequence is prominent either near the vernal or the autumnal equinoxes. Many recurring sequences do not show such varintions, so the question arises of the possibility of the apparent variations being caused by chance. SHAPIRO (1967) with a power spectrum analysis of the geomagnetic index $C_{i}$ over the period 1884 to 1964 has concluded that any annual modulation of the 27-day variation in this index is either nonexistent or very small in magnitude. Thus it seems safe to conclude that any annual variation in recurring geomagnetic storms related to the axial hypothesis is very small at best. 
NESS (1965) has shown that the direction of the geomagnetic axis is an important influence on the topology of the magnetosphere and geomagnetic tail. This is consistent with the equinoctial hypothesis.

\section{SOLAR ORIGIN OF THE INTERPLANETARY SECTOR PATTERN}

The interplanetary sector pattern observed by IMP-I and shown in Figure 3 has been compared with the direction of the photospheric magnetic field as observed with the solar magnetograph (BABCOCK, 1953) at the Mount Wilson Observatory. The photospheric observations were obtained by Dr. Robert Howard. From the individual solar magnetograms obtained each day (weather permitting), synoptic charts are prepared (Figure 48). The solid contours indicate field directed out of the sun and the dashed contours indicate field directed into the sun; the contours levels represent 4, 8, 16 and 24 gauss. The synoptic charts show large regions of relatively weak magnctic fields. Synoptic charts for two successive solar rotations show much similarity, indicating that many of the features have a lifetime that is at least comparable with the solar rotation period. The large but localized magnetic fields of sunspots do not appear on these charts, and discrete events such as flares also are not recorded (NESS and WILCOX, 1966).

For comparison with the interplanetary sector structure, a particular heliographic latitude is selected, and the predominant direction of the photospheric field in each 12-hour interval is recorded. Details of the procedure are given by NESS and WILCOX (1966). A crosscorrelation as a function of time lag can then be computed between the direction of the photospheric magnetic field at a given latitude and the interplanetary sector structure. Result: 
for $25^{\circ} \mathrm{N}, 20^{\circ} \mathrm{N}$, . . $0^{\circ}$ solar latitudes with respect to the visible disk are shown in Figure 49. A prominent positive peak at a lag of approximately $41 / 2$ days is evident. A peak in the correlation at a lag of $41 / 2 \pm 1 / 2$ day would correspond to an average radial solar wind velocity of $385 \pm 45 \mathrm{~km} / \mathrm{sec}$. The average radial solar wind velocity observed by the Massachusetts Institute of Technology plasma detector on IMP-1 was $360 \mathrm{~km} / \mathrm{sec}$ (PAI et al., 1967) ${ }^{*}$ and the average solar wind velocity obtained by the Ames Research Center plasma detector on IMP-I was $378 \mathrm{~km} / \mathrm{sec}$ (WOLFE et al., 1966). Thus the observed lag from the central meridian passage of a photospheric feature to the time of observation near the earth was consistent with the time for convection of a magnetic feature by the solar wind from the solar atmosphere to the orbit of the earth. The IMP-I observations were made at a time when the sector structure was changing very little with time, so that a quasi-stationary situation could be established in the solar atmosphere. It seems possible that if a new photospheric feature appears there could be a delay of several weeks until the level of the solar atmosphere that is the source of the interplanetary sector pattern has received the change in magnetic pattern.

*The preliminary MIT average velocity quoted by NESS and WILCOX (1966) was somewhat lower. 
The latitude of the solar source of the interplanetary sector pattern cannot be determined accurately from the crosscorrelation analysis for the following reason. Inspection of Figure 48 shows that the pattern of the photospheric magnetic field at this time is dominated by regions of a single polarity (into or out of the sun) with an extent in both latitude and longitude covering an appreciable fraction of the solar disk. Note that a region often extends on both sides of the solar equator. Because of the predominance of these large regions it is reasonable that the crosscorrelation curves for several solar latitudes shown in Figure 49 should display similar peak correlations. Therefore the differential rotation of the photospheric magnetic field was investigated, since the recurrence period of the interplanetary sector pattern should be the same as the average rotation period of the latitudes of the solar source. Figure 50 shows autocorrelations of the direction of the photospheric magnetic field for latitudes at the center of the visible disk and $25^{\circ} \mathrm{N}$ thereof. The increase in period caused by the differential rotation is quite apparent. From autocorrelations similar to those of Figure 50 the rotation period of the photospheric magnetic field as a function of latitude is computed, and the result is shown in Figure 51. The solid line is the rotation period determined by NEWTON and NUNN (1951) from analysis of long-lived sunspots. The arrows at the left of Figure 51 indicate the recurrence period of the interplanetary sector pattern and the uncertainty therein. The comparison in Figure 51 of the interplanetary field recurrence period with the differential rotation of the photospheric field suggests that the latitude of the photospheric source of the interplanetary field was within $10^{\circ}$ or $15^{\circ}$ of the center of the visible disk. These results indicate that during the time interval observed by IMP-1 
some of the magnetic field lines passing through the photosphere near the center of the visible disk tended to be transported out by the solar wind plasma to become part of the nearby interplanetary magnetic field.

A further investigation of the heliographic latitude of the IMP-1 interplanetary sector pattern was done by WILCOX and NESS (1967). Figure 52 shows an autocorrelation of the direction of the photospheric field as a function of latitude at intervals of $5^{\circ}$ for seven solar rotations centered on the three rotations observed by IMP-1. The format of Figure 52 is as follows: for the autocorrelation for $35^{\circ} \mathrm{N}$, the top line represents an autocorrelation value of 1.0, the horizontal labeled $35^{\circ}$ represents a value of 0.0 , and the next lower line (labeled $30^{\circ}$ ) represents a value of -1.0 . Within these limits the autocorrelation for $35^{\circ}$ is shown approximately centered about the horizontal line labeled $35^{\circ}$. Each other latitude is overlaid using the same format. We can observe in Figure 8 that the IMP-1 sector structure gives a characteristic signature in an autocorrelation in the form of a recurrence peak at about 27 days, together with a centered secondary maximum surrounded by the two minima. In Figure 52 the photospheric autocorrelation for $15^{\circ} \mathrm{N}$ is a good approximation to this signature of the interplanetary sector structure, and the autocorrelations for $10^{\circ} \mathrm{N}$ and for $20^{\circ} \mathrm{N}$ also display a fairly prominent representation of this signature. At $5^{\circ} \mathrm{N}$ and $25^{\circ} \mathrm{N}$ only a slight suggestion of this signature appears, while at $0^{\circ}$ and $30^{\circ} \mathrm{N}$ the signature has completely disappeared. This suggests that heliographic latitudes between about $10^{\circ} \mathrm{N}$ and $20^{\circ} \mathrm{N}$ had a large scale structure most similar to the interplanetary sector structure observed by IMP-1, which is consistent with results obtained in Figure 5l. It should be noted that the average heliographic latitude of the earth (and of IMP-1) during these interplanetary observations was $31 / 2^{\circ} \mathrm{S}$. 
HOWARD (1959) has shown that photospheric magnetic features have a close relation to calcium plage regions, such that plages are outlined very nearly by a 10 gauss contour line. It would therefore be of interest to compare the results derived from photospheric magnetic field observations with an analysis of the location of calcium plages at this time. Figure 53 shows a superposition of calcium plage locations obtained from several Fraunhofer Institute maps of the sun on days when the large sectors shown in Figure 3 would be expected to be approximately centered at central meridian (for details see WILCOX and NESS, 1967). The leading edge of a sector should be at about $50^{\circ} \mathrm{W}$ and the trailing edge of a sector at about $50^{\circ} \mathrm{E}$ longitude. The densest concentration of plages occurs in the latitude range $10^{\circ} \mathrm{N}$ to $20^{\circ} \mathrm{N}$, which was shown above to be the region most similar to the interplanetary sector structure. In this range of latitudes the preceding boundary of the sector $(500 \mathrm{~W})$ is relatively free of plages, while the densest concentration of plages occurs approximately one quarter of the distance into the sector. In the trailing portions of the sector there are considerably fewer plages. This variation of plage density as a function of position within the sector is qualitatively similar to the results obtained for the interplanetary field magnitude and solar wind velocity shown in Figures 10 and 11.

\section{North-South Asymmetry}

The fact that the average heliographic latitude of IMP-1 was $31 / 2^{\circ} \mathrm{S}$, whereas the solar structure most similar to the interplanetary sector structure was at latitudes around $10^{\circ} \mathrm{N}$ to $20^{\circ} \mathrm{N}$, requires an explanation. One possibility is a north-south esymmetry in the flow of the solar wind. WILCOX (1965) suggested that the observation by BELI (1961) that "northern flares of a given type are far more likely to produce significant geomagnetic disturbances than are corresponding southern flares" might be understood on the basis of an asymmetric 
solar wind flow, such that even for quiet sun events the earth could tend to be connected to northern solar regions. The larger solar activity in the northern hemisphere would cause a greater coronal heating and temperature in the north as compared with the south, and it would be expected (PARKER, 1963) that a greater efflux of solar wind would occur. The resulting inbalance in lateral pressure between larger solar wind efflux in the north and a smaller efflux in the south might provide a qualitative explanation for a north-south asymmetry in the flow of the solar wind.

Some authors (see for example KRIVSKY, 1965) have attributed the northsouth asymmetry to a motion of the sun and solar system relative to the interstellar material in the local galactic neighborhood. Such a motion could produce an asymmetry in the outer boundary shell of the solar wind, but the supersonic character of the solar wind flow for hydromagnetic waves would appear to prevent an asymmetry from this cause in the inner solar system. Information about possible boundary asymmetries cannot flow upstream in the solar wind, since a typical solar wind plasma velocity is $400 \mathrm{~km} / \mathrm{sec}$ and a typical Alfvén velocity in the solar wind is $70 \mathrm{~km} / \mathrm{sec}$.

The observation that the solar source of the interplanetary sector pattern was in the range of about $109 \mathrm{~N}$ to $20^{\circ} \mathrm{N}$ is not consistent with the axial hypothesis discussed earlier as an explanation for the semiannual maxima in geomagnetic activity. If the solar source was in these northern latitudes when the average heliographic latitude of the spacecraft was $3^{\circ} \mathrm{S}$, it would not appear that the $7^{\circ}$ tilt of the solar axis with respect to the ecliptic would have a determining influence on geomagnetic activity. 
Longitudinal Extent of the Solar Source of a Sector

An important question that arises in the consideration of the solar source of the interplanetary sector structure has to do with the longitudinal extent on the sun of the region that is the source of a sector. One possibility is the "nozzle" hypothesis, in which a localized area on the sun is responsible for the magnetic field of a sector, with the field from a localized source expanding very much in longitude such as to fill an entire sector. Another possibility could be called the "mapping" hypothesis, in which a sector occupying, for example, $100^{\circ}$ in longitude is assumed to have a source region on the sun which also occupies approximately $100^{\circ}$ of longitude. When the Mariner 2 observations in 1962 yielded the surprising result that for approximately half a solar rotation the interplanetary field near the earth was directed away from the sun, DAVIS (1965, p. 204) suggested that this feature could be understood if the magnetic lines for the entire sector arose from a small region on the sun in which the field direction was essentially unidirectional. Davis suggested that the solar wind may not well-up from the lower corona uniformly over the entire surface of the sun, but that its flow is inhibited by the magnetic field in most places. Only where the fields are unusually regular, weak and radial out to large distances over substantial areas can the gas well-up. These areas might feed the entire upper corona with material for the solar wind. At larger radii the wind dominates the magnetic field and sweeps it out as in the usual model. Thus all the gas in the solar wind would come from a few regions in the photosphere, and the radial component of the field in interplanetary space would reverse sign infrequently. 
The mapping hypothesis, in which there is a tendency for each longitude near the sun to be connected by interplanetary field lines to a longitude at the orbit of the earth, is to some extent supported by the observations of energetic particles. A theoretical Archimedes spiral interplanetary field line through the earth would originate in the solar disk at about $60^{\circ} \mathrm{W}$ longitude. Solar flares in this vicinity are often observed to produce energetic particles near the earth with delay times not very different from direct transit times for these particles along the interplanetary field line. Flares in the eastern portion of the solar disk often produce energetic particles observed near the earth after a longer delay. MC CRACKEN et al. (1967) conclude that such eastern flares produce cosmic rays that experience diffusion in the vicinity of the sun until they reach the tube of force upon which the observing spacecraft was located. ANDERSON and IIN (1966) draw similar conclusions from observations of the propagation of solar flare electrons of energy greater than $40 \mathrm{kev}$.

The crosscorrelations shown in Figure 49 between the direction of the photospheric field and the interplanetary sector structure favor the mapping hypothesis, because if the polarity corresponding to an entire sector were significant in only a small portion of solar longitude the crosscorrelation, which gives equal weight to all intervals of longitude, would not show a significant positive peak. On the other hand we cannot formally exclude the possibility that from a localized source in the sun unidirectional photospheric fields diffuse out such as to produce the observed correlations. Figure 53 suggests that the probability distribution of plages as a function of position within a sector is similar to the distribution within a sector of the magnitude of the interplanetary field and of the solar wind velocity. This is consistent with the mapping hypothesis. It is the opinion of the author that the mapping 
hypothesis is the most reasonable one, although further observational evidence on this point would be welcome.

\section{Sectors Contain M-Regions?}

We present in the following sections some evidence for answering the above question with a yes. BARTELS (1932) noted that many sequences of recurrent geomagnetic activity could not be identified with any obvious source on the sun, and proposed the name M-region for the unknown solar source of recurrent geomagnetic activity. Many papers since that time have been addressed to this question, but it still has no generally accepted answer. We suggest here a certain change of emphasis. The prominent recurring geomagnetic storms and their M-region sources on the sun may be only a particular case of a more-orless continuous relation between certain solar structures and the condition of the geomagnetic field and radiation belts, with the interplanetary medium serving as the transmission medium between the two. It is easy to understand why so much attention has been given to the recurring maxima of geomagnetic activity. On a casual inspection of the Bartels 27-day charts of geomagnetic activity these are the features that stand out most prominently. At the time not too long ago when interplanetary space was thought to be essentially a vacuum with only a few streamers extending out from the sun to the earth, it was natural to investigate the origin of such streamers and not give much attention to other portions of the sun. Also, from a practical viewpoint, 
geomagnetic activity is associated with aurora and disruption of communications. We propose that at least in a period of quiet sun such as observed by IMP-I a more or less continuous relation exists between solar structure and geomagnetic response. We assume that the mapping hypothesis discussed above is more nearly correct than the nozzle hypothesis. (On a local scale the magnetic field in the lower corona may be of great importance in guiding and channeling the flow of the solar wind, but we assume that on a distance scale appropriate to a sector the mapping hypothesis is more appropriate.)

\section{Structure of the Photosphere}

Let us first examine what information about the structure of the solar source of the sector structure might be gained from photospheric observations. The photospheric magnetic field can be observed using photoelectric detection of the Zeeman effect. The entire solar disk or a portion thereof can be scanned, using the solar magnetograph (BABCOCK, 1953). BUMBA and HOWARD (1965) have used this instrument to study the distribution of weak magnetic fields on the solar surface during a period of $41 / 2$ years, using resolution of 23 seconds of arc. A slowly changing pattern of background fields with a magnitude of a few gauss is observed. In the period near solar activity minimum, active regions are concentrated in complexes of activity whose location and development are clearly defined. The largest of these complexes, consisting of many active regions, result in the formation of unipolar magnetic regions (UMR).

We propose that the background field pattern observed by Bumba and Howard related to may $b^{v}$ the solar source of the interplanetary sector pattern. Some of the observed features of the background fields are listed, together with comments related to the interplanetary sector structure: The magnetic fields of the two polarities are distributed with striking regularity in longitude across 
the solar surface. (This is consistent with the unidirectional characteristic of the sector structure.) Although many features exhibit some symmetry about the equator, in general the center of gravity of these fields is slightly to the north of the equator. (This may be related to the suggestion drawn from Figure 52 that the heliographic latitude of the solar source of the sector structure was at heliographic latitudes of about $10^{\circ} \mathrm{N}$ to $20^{\circ} \mathrm{N}$.) In general, the main characteristics of the distribution of background fields can be traced for approximately one year. (This is consistent with the evolution of the sector structure shown in Figure 13 up to the end of 1964, which was the interval studied by Bumba and Howard.) Usually old features are joined by new features of like polarity to maintain the general appearance of the pattern. In general, the disappearance of background fields takes place as a gradual weakening of fields over a large area. There is no characteristic motion associated with this disappearance. (This could be related to the toward sector shown in Figure 13, which was probably quasi-stationary for well over a year and then disappeared between Bartels rotations 1798 and 1799.)

Bumba and Howard observed that in 1959 at least $50 \%$ of the area of the solar surface was occupied by measurable fields, while in 1962-1964 (the period observed by spacecraft) the background fields were weaker and occupied typically $20 \%$ of the solar surface. (We suggest that even in 1962-1964 a regular solar pattern which was the source of the sector structure may have remained at almost all longitudes, but that the magnitude of the photospheric fields associated with the pattern was so small that only about $20 \%$ of the area could be observed with the solar magnetograph.)

Bumba and Howard report that the development of a single active region is not enough to make a permanent change in the pattern of the weak background fields. To achieve the slow transitions from one form to another that they 
observe in the background fields, it is necessary to maintain a certain density of activity over a large area. (This is consistent with the viewpoint that the sector pattern is usually not related to any single active region. However the probability of development of an active region, and the form and complexity of the region and its sunspots may be related to position within the structure of the background fields.)

\section{Unipolar Magnetic Regions}

Bumba and Howard define a unipolar magnetic region (UMR) as being a large region of one polarity (always the following polarity in each hemisphere), generally stretching poleward of the pattern of background fields, and often extending more than $100^{\circ}$ in longitude. The magnetic fields are generally strongest in the leading portions and become weaker in the following tail. (This is approximately the same as the change of interplanetary field magnitude within a sector as shown in Figure 10.) The same is true of the calcium emission which is seen in the UMR. (Figure 53 indicates that calcium plages are most likely to occur in the preceding portion of a sector.)

BUMBA and HOWARD (1965) further define a "ghost" unipolar magnetic region as being a region of leading (for that hemisphere) polarity that is roughly the same shape as a UMR and sometimes nearly as large, but that has fields in the tail that are more than a factor of 2 weaker than the UMR. There is only slight enhanced calcium emission to be seen accompanying these "ghost" UMRs. Almost always during the lifetime of the regions a large quiescent prominence separates the UMR from the ghost UMR. It seems possible that the interplanetary sector pattern observed by IMP-1 and shown in Figure 3 had solar structures resembling UMRs (or at least the low-latitude extention of UMRs) as sources of 
the toward sectors, and solar structures resembling ghost UMRs (or their lowlatitude extensions) as sources of the away sectors, as has been suggested by SAKURAI (1966a, b) (remember that the solar source appeared to be in the northern hemisphere according to the analysis associated with Figure 53). WIICOX and NESS (1965) have explicitly identified a ghost UMR as being associated with the source of the away sector extending from 2 December to 12 December in Figure 3. In the synoptic chart of Figure 48 the observed boundaries of this sector are indicated, assuming a uniform radial solar wind velocity and using the measured plasma velocity from the Massachusetts Institute of Technology detector on IMP-1* . The source of the away interplanetary sector of Figure 3 is readily identified in the photospheric synoptic chart of Figure 48 as being associated with a ghost UMR (solid-line contours), as defined by BUMBA and HOWARD (1965). BUMBA and HOWARD (1966) have examined the correlation of UMRs with recurrent geomagnetic storms. Figure 54 shows the Bartels diagram of geomagnetic activity for the last several years, and beside it on the same scale a diagram which marks the central meridian passage of UMRs (taken from Table 2 of BUMBA and HOWARD, 1965). There is a correlation between the two sets of data in Figure 54 in the sense that the UMRs come to the central meridian several days after the commencement of recurrent geomagnetic storms. This is consistent with the suggestion by WIICOX and NESS (1965) that the ghost UMR was associated with the source of the positive sector responsible for recurrent geomagnetic disturbance; since the associated UMR

*Figure 48 was drawn using preliminary MIT values for solar wind velocity. The final values (PAI et al., 1967) would move the preceding boundary of the sector about $1 / 2$ day to the right (later) in Figure 48. 
follows the ghost UMR on the disk it would be expected that the UMR would come to central meridian several days after the commencement of the recurring geomagnetic storm. The suggestion we make here is that in addition to the UMR-interplanetary sector relationships that are prominent enough to be readily identified (i.e. have a sufficiently large amplitude), the other sectors of Figure 3 are perhaps to be identified with similar solar structures which are of smaller amplitude and therefore not readily identified with the solar magnetograph. BUMBA and HOWARD (1965) recognized the possibility that there is a background field pattern present that is weaker than the limit of the magnetograms. They also point out that in the future in connection with solar observations it will be extremely important to associate the position of the observations with respect to the weak background fields. For example, it will not be sufficient simply to say that an observation was made in the center of the disk outside active regions. The presence, intensity, and structure of the background fields may change the physical conditions in the area enough to alter the results of these observations.

It may be that prominent recurring geomagnetic activity sequences are associated with the preceding portion of a sector that is unusually effective in producing geomagnetic response, but that all sectors tend to produce geomagnetic activity as the preceding portion rotates past the earth, as is shown for the IMP-I observations by Figures 34 and 35. MUSTEL (1967) has pointed out that in some cases noticeable geomagnetic activity does not fill in the whole sector. In the present interpretation this is just what would be expected, since it is only the preceding portion of the sector that has larger-thanaverage geomagnetic activity. We suggest that the fundamental consideration is the sector as an entity having an internal structure, and not just those 
days on which geomagnetic activity is sufficiently large to show recurring sequences of activity.

Chree and Stagg--Superposed Epoch Analysis

The analysis of CHREE and STAGG (1927) utilizing 20 years of observations of the geomagnetic character figure appears to be consistent with and support the viewpoint herein adopted. Their results are

show in Figure 55; their method is as follows. The five most disturbed days in each month are chosen as the zero days in a superposed epoch analysis, and the values of the geomagnetic character figure for these five most disturbed days are entered at position zero. Then the value of the geomagnetic character index 27 days later, 54 days later . . and 27 days previously, 54 days previously . . are entered at the appropriate position. Finally the values at each time position are averaged together, and the results are plotted in the top portion of Figure 55. A similar analysis is performed for the five most quiet days of each month, and the results are plotted in the bottom portion of Figure 55. Since the zero day is unusually disturbed (or quiet) the average character figure on that day is naturally very large (small). But the analysis shows further that significant peaks (valleys) are found at intervals of 27 days up to at least 108 days before and after the primary pulse. This analysis by Chree and Stagg gave the clearest evidence for the recurrence tendency of geomagnetic activity, since essentially no selection is made of the data to be used, and no preconceived notion about the recurrence interval is introduced into the analysis. The principal point of interest for the present discussion is the conclusion of Chree and Stagg from Figure 55 that, relative to the primary pulse, the secondary valleys related to the most quiet days are quite 
as prominent as the secondary peaks related to the most disturbed days. In other words, the recurrence tendency is equally prominent for disturbed conditions and for quiet conditions. This is consistent with the picture discussed in the present paper in which sectors having the internal structure shown in Figures 10, 11 and 12 are rotating past the earth and producing geomagnetic activity. The most disturbed recurrences of Figure 55 would correspond to the preceding portions of sectors, and the most quiet recurrences of Figure 55 would correspond to the trailing portions of sectors.

Chree and Stagg interpreted Figure 55 as indicating that there is no net tendency for a recurring geomagnetic sequence to decrease (or increase) in amplitude with the successive recurrences after the first. (A symmetric variation with time is allowed, however. For example, a recurring sequence could increase in amplitude up to the midpoint of the recurrence and then decrease in amplitude in a symetric way, or vice versa.) Chree and Stagg discuss Figure 55 as follows: "It may be well to point out that the increase from left to right in the amplitude of the previous secondary pulses and the corresponding decrease in the subsequent pulses represents not a waxing and waning in the amplitude of the disturbance but the lesser probability of two recurrences than of one, of three recurrences than of two, and so on. If we suppose a disturbance recurring in 27 days of sufficient intensity to figure as a disturbed day on five successive occassions, it will contribute every time to the primary pulse. The first occurrence contributes to all the previous pulses, the second to the pulses centering at $-81,-54,-27$, and +27 days, the third to the pusles centering at $-54,-27,+27$ and +54 days, the fourth to the pulses centering at $-27,+27,+54$ and +81 days, and the fifth only to the subsequent pulses. Supposing the character figure to decline steadily, then each previous pulse would receive a larger contribution than 
the corresponding subsequent pulse. A tendency to wane would show itself in a diminished amplitude of each subsequent positive pulse as compared with the amplitude of the corresponding previous positive pulse. If we take the allyear results represented in Figure 55 as being the least exposed to accidental features, we see no decided tendency for the subsequent pulses to be either less or greater than the previous. If the positive pulse centering at +27 days slightly exceeds that centering at -27 days, the positive pulse centering at +108 days is less than that centering at -108 days." If we associate the sector patterns with the recurrent features studied by Chree and stagg, we have the result that on the average a new sector does not begin with a large amplitude and subsequently decreases, but rather that there is no net tendency for the amplitude to either decrease or increase throughout the recurrent series.

\section{Extent in Latitude of the Solar Source}

We have suggested that the interplanetary sector structure may have a solar source in the background photospheric magnetic field patterns which have sufficiently small magnitudes that the pattern is marginally detected with the solar magnetograph. We can consider now the extent in heliographic latitude of the postulated solar pattern. The interplanetary sector patterns shown in Figure 13 and the associated recurring geomagnetic disturbances have an almost precisely 27-day recurrence period up to the end of 1964 (we shall consider later the situation to be expected in the new solar cycle when solar activity is increasing). The analysis of NESS and WIICOX (1966), part of which is shown in Figure 49, indicates that significant 
correlations can be observed between the interplanetary sector pattern and the direction of photospheric fields over a considerable range in latitudes.* We must consider how the differential rotation would affect the solar sector structure. As observed from the earth the solar equator has a rotation period of about 26.9 days, and a latitude of about $25^{\circ}$ has a rotation period of about 27.9 days. Let us assume for purposes of illustration that the sector pattern shown in Figure 3 existed on the sun at a given instant of time invariant with latitude, i.e. that at all latitudes the heliographic longitudes of the sector boundaries were identical. Consider then the situation one year later. The more than 13 solar rotations during the year would cause the central meridian appearance of a sector boundary at $25^{\circ}$ to lag the central meridian appearance of the same boundary at the equator by more than 13 days. A crosscorrelation between the interplanetary sector pattern and the photospheric field direction at $25^{\circ}$ should then produce a peak at a position more than 13 days displaced from the similar peak to be obtained with a crosscorrelation of the photospheric field direction at the solar equator. But the crosscorrelations obtained by NESS and WILCOX (1966) showed the position of the peaks in such correlations to be very similar. (We can cite the cosmic ray analysis of MORI et al. (1964) shown in Figure 29 as evidence that the four-sector pattern observed by IMP-1 had indeed existed for almost a year before the IMP-1 observations.) This suggests that the solar pattern that

*The IMP-I analysis included approximately three solar rotations. An extended analysis using the interpolated field for 1964 as shown in Figure 13 is presently in progress. 
is the origin of the interplanetary sector structure may have approximately a 27-day recurrence time in heliographic latitudes from at least about $30^{\circ} \mathrm{N}$ to $30^{\circ} \mathrm{S}$. This could be related to the conclusion by KIEPENHEUER (1953) that the internal cause of sunspots has a meridional structure that is not affected by the differential rotation of the sun. Similar conclusions have been reached in other investigations, such as GUSS (1964) and WARWICK (1965)* DODSON-PRINCE and HEDEMAN (1967) have also suggested that there may be a pattern of formation of active centers which is not affected by differential rotation.

Possible Solar Structure at a Sector Boundary

A plausible magnetic field pattern for the large-scale field in the solar atmosphere associated with a sector boundary is shown in Figure 56, which is modified (to include the asymmetry between the preceding and following portions of sectors) from a suggestion by BILLINGS and ROBERTS (1964) concerning the solar source of M-Region geomagnetic storms. The rate of expansion of the solar wind will be influenced by the temperature near the region where the flow changes from subsonic to supersonic. The isothermal surfaces are depressed above the sector boundary because the thermal conductivity normal to the magnetic field is negligible compared to that along lines of force. The pattern of the solar source of the sector structure (such as perhaps Figure 56) does

*A discussion by WILCOX and SCHATTEN (1967) indicates that this investigation may not have had a unique statistical interpretation; the physical interpretation made by Warwick appears to be consistent with the present viewpoint. 
not include the many magnetic lines that loop around at relatively low elevations and return to the photosphere, giving rise to the many small regions of mixed polarity observed with the solar magnetograph.

More observations are needed to clarify the solar structure corresponding to a sector boundary. It seems possible that both the solar magnetic field configuration and the structure of the photospheric velocity fields that may serve as the source of coronal heating could be important in producing the interplanetary sector structure.

Solar Source of the Interplanetary Filament Structure

We have discussed above some possibilities with regard to the solar source of the large-scale sector pattern. Now we consider the source of the interplanetary filaments that were discussed in a previous section. A schematic picture is shown in Figure 57. MICHEL (1967) has suggested that the gross solar wind modulation is represented by granules $3 \times 10^{3} \mathrm{~km}$ in diameter and having lifetimes of about 5 minutes. It appears to the author that the supergranulation pattern observed by SIMON and LEIGHTON (1964) might play a more prominent role as a source of interplanetary field structure. The supergranulation is observed in the photosphere and has an average diameter of $32,000 \mathrm{~km}$. Within each supergranule the observed flow pattern is radially outward, and local maxima of photospheric field are observed at the boundaries. The lifetime of the pattern is about 20 hours.

Perhaps further discussion should be deferred until the solar origin of the interplanetary filaments has been studied further. It seems possible that a rather detailed map of the solar surface is presented as the interplanetary field structure rotates past the earth. 


\section{Solar Magnetograph Observations}

ALFVÉN (1966) and STENFLO (1966) have pointed out that the filamentary structure of the solar corona, the prominences and the chromosphere may be produced by electric currents, which cause a bunching of the magnetic lines of force. Most of the magnetic field may then be confined to the filaments, inside which the field strengths may be orders of magnitude greater than in the surroundings. Since the entrance slit of the solar magnetograph is larger than the size of the filamentary structure, it is possible that the magnetograph could produce an incorrect observation of the field strength. * The spacecraft observations discussed here appear to be consistent with the solar magnetograph observations (at least in the equatorial latitudes) with regard to large-scale field magnitude and direction. The average value of the radial component of the interplanetary field at the orbit of the earth as observed by several satellites is about $3 \times 10^{-5}$ gauss. This component should scale as $1 / R^{2}$ which would yield a corresponding photospheric field of about I gauss, which is approximately the average magnitude observed with the solar magnetograph. Also the crosscorrelations shown in Figure 49 between the direction of the photospheric field and the interplanetary field suggest that at least on this scale the solar magnetograph is observing the correct direction of the photospheric field. The correlations of Figure 49 have been obtained at equatorial latitudes. The magnetic field in the polar regions of the sun

*The author, who has enjoyed the kind hospitality of both the Royal Institute of Technology in Stockholm and the Mt. Wilson and Palomar Observatories in Pasadena, has followed this discussion with some interest. 
is of the most direct interest for Alfvén's theory, which assumes that there is a dipole-like field within the sun, and that disturbances near the core propagate to the solar surface as hydromagnetic waves and produce sunspots (ALFVirn, 1956). The spacecraft observations discussed above apparently do not give any direct information about the polar field of the sun.

\section{Azimuthal Velocity of Solar Wind Plasma}

The discussion in this review has often concerned the corotation of the interplanetary magnetic field pattern, by which is meant the tendency for a magnetic feature such as a sector boundary to corotate with the sun and to appear at the earth every 27 days. The only material velocity involved in this approach is the approximately radial flow of the solar wind plasma which convects magnetic features away from the sun. We now discuss a completely different matter, the possibility for an azimuthal velocity component in the flow of the solar wind plasma. In the lowest levels of the corona where solar magnetic field strengths are large and the energy density of the field may exceed the energy density of the plasma, the solar field may cause the coronal plasma to rotate almost as a solid body with the sun. At the orbit of earth the energy density in the streaming solar wind plasma is nearly two orders of magnitude larger than the energy density of the interplanetary magnetic field, and the flow velocity of the solar wind is nearly radial. BRANDT (1967) has made an extensive study of the velocity of the solar wind plasma as deduced from observations of type 1 (ionized) comet tails, and finds evidence for a mean tangential component of the solar wind velocity near the earth of approximately $10 \mathrm{~km} / \mathrm{sec}$ directed in the sense of the solar rotation. HUNDHAUSEN et al. (1967c) have observed the solar wind velocity with a plasma detector on the 
Vela 2 satellite during the period July 1964 - July 1965. They find that the average direction of flow for every solar rotation during this period is from east of the sun; the average flow over the entire year is from approximately $11 / 2^{\circ} \mathrm{E}$ of the sun, corresponding to a mean nonradial velocity component in the ecliptic plane of approximately $10 \mathrm{~km} / \mathrm{sec}$. This angular momentum of the solar wind corresponds to an appreciable torque on the sun. Theoretical discussions have been given by DICKE (1964), PNEUMAN (1966), WEBER and DAVIS (1967), MODISETTE (1967), and FERRARO and BHATIA (1967). The torque on the sun can be calculated assuming the coronal plasma to rotate as a solid body with the sun out to a distance at which the Alfvén wave velocity using the radial component of the field is equal to the flow velocity of the plasma. This is a formal statement; it does not mean that the coronal plasma is actually rotating as a solid body out to this distance. BRANDT (1966) has pointed out that this loss of angular momentum to the solar wind could be important in the evolution of the sun. It is possible that the sun formerly was rotating with a much shorter period than is observed at the present time, and that the fast-rotating solar core (period approximately 2 days) proposed by DICKE and GOLDENBERG (1967) retains this large rotational velocity, while the outer layers of the sun have been slowed down by the loss of angular momentum to the solar wind plasma and magnetic field.

Quiet Regions of the Sun are Independent of the Sunspot Cycle?

BASLER (1966) has found that by arranging solar data according to when quiet periods are observed on the earth, a component of solar activity is selected which does not show the usual tendency to follow the 1l-year sunspot cycle. Figure 58 is a superposed epoch diagram (the same method of analysis as used by Chree and Stagg in Figure 55) in which the zero day corresponds 
to large absorption of cosmic radio noise at College, Alaska, and the ordinate is the daily solar radio flux at $2800 \mathrm{Mc} / \mathrm{s}$. Figure 58 shows an overall decrease in solar activity from left to right which, as would be expected, corresponds very closely to the average decline in activity during the $51 / 2$ year period examined. Figure 59 is the same kind of analysis except that zero day corresponds to a low absorption of cosmic radio noise. The curve in this figure is essentially horizontal; it does not show the decrease in solar activity from left to right which was seen in Figure 58. The same gross features were also found by Basler in an analysis using other daily solar indices including the American relative sunspot number, the $5303 \AA$ coronal index integrated over the whole visible disk, an index of flare area, a flare activity index, and the flux at $200 \mathrm{Mc} / \mathrm{s}$. In 1962, a few years after solar maximum, the Mariner 2 satellite observed several recurring solar wind streams in which the maximum velocity was of the order of $600 \mathrm{~km} / \mathrm{sec}$. In the intervals between the streams, however, the solar wind velocity was as $10 \mathrm{w}$ as $307 \mathrm{~km} / \mathrm{sec}$ and the temperature was approximately $3 \times 10^{4} \mathrm{O}_{\mathrm{K}}$ (NEUGEBAUER and SNYDER, 1966). HUNDHÁUSEIN et al. (1967c) observed the solar wind velocity with a Vela 2 satellite near solar minimum during the period July 1964 - July 1965 and found that during quiet periods the solar wind velocity was about $320 \mathrm{~km} / \mathrm{sec}$ and the temperature was a few $\times 10^{4}{ }^{\circ}$. BARTELS (1963) pointed out that in the 30 years of geomagnetic data examined no month passes without a few quiet days; there occur always at least five days in each calendar month on which the level of disturbance is of the order of $K_{p}=2^{+}$or lower. All of these observations suggest that there are quiet regions of the sun whose physical characteristics do not change appreciably during the 11-year sunspot cycle. This suggests that the physical characteristics in the trailing portion of the sector pattern may be reasonably stable through the solar activity cycle. 


\section{SUMMARY}

This review has dealt for the most part with observations in the period 1962-64 during the decline of solar activity. The spacecraft observations clearly give valuable new information on solar-terrestrial physics, but it must be considered that they cover only a very short interval in terms of even one solar cycle, and that some of the conditions observed may be anamolous when compared with the average conditions over many solar cycles.

\section{Interplanetary Medium}

The large-scale structure of the interplanetary medium was dominated by a sector pattern in which the interplanetary field was predominantly away from the sun for several consecutive days and then predominantly toward the sun for the next several days. The sector pattern corotates with the sun such that a particular feature such as a sector boundary is observed near the earth approximately once every 27 days. The average sector has a coherent internal structure in which the magnitude of the field and the solar wind velocity rise to a peak in the preceding portion of the sector and decline in the following portion. Cosmic rays tend to corotate with the interplanetary field structure. In addition there may be differential flows toward the sun within some sectors and away from the sun in other sectors. The internal structure of an average sector may also include a differential flow of cosmic rays outward near the preceding portion of the sector and inward near the following portion of the sector.

During the decline of the solar cycle the sector pattern appeared to be quasi-stationary over a year or longer, and to show an approximately 27-day 
recurrence period. Some rather abrupt changes in the sector pattern appeared to mark the influence of the new solar cycle, in which the evolution of the sector pattern became more rapid and the recurrence period changed to approximately 28 days.

A prominent smaller-scale feature within the sectors are filaments which appear to connect to particular regions in the lower solar atmosphere. Several interplanetary parameters may change rather abruptly at the boundary of a filament, such as the direction and magnitude of the interplanetary field and the solar wind velocity, density and temperature. Solar cosmic rays produced by flares often appear to be guided along such filaments.

Geomagnetic Activity and Radiation Belts

As the sector pattern rotates past the earth profound changes are observed in geomagnetic activity and in the structure of the radiation belts. On the average, geomagnetic activity appears to reach a peak in the preceding portion of the sector and to decline in the following portion with a structure somewhat similar to that observed for the interplanetary field magnitude and solar wind velocity. The particular density in the outer radiation belts appears to increase near the sector boundaries, and the regions of increased density then drift or diffuse inward. Features of the geomagnetic field such as pearl fluctuations appear to change in an organized manner as the sector rotates past the earth. The prominent recurring geomagnetic disturbances that have been associated with M-Region sources on the sun are here interpreted as a special case of the recurring sector pattern in which the amplitude of the disturbance in the preceding portion of the sector is larger than usual. 


\section{Solar Sector Structure}

The source of the interplanetary sector structure may be associated with the pattern of the weak photospheric background fields observed with the solar magnetograph. The occurrence of active regions may be more probable near the preceding portions of the sector pattern. The solar source structure may be relatively independent of the differential rotation in equatorial latitudes.

The discussion of this review has dealt with the quasi-stationary or slowly changing large-scale features of the interplanetary field. Transient effects associated with solar flares or other disturbances have not been discussed, and are presumably to be regarded as perturbations to the patterns discussed here.

We have discussed some modern views of the nature of the sun and the interplanetary space. We can conclude the discussion by examining a Roman mosaic in Egypt shown in Figure 60 (HERDEG, 1962). The caption for this mosaic was as follows: "Detail of a Roman mosaic pavement from Egypt. The hub of the sun, from which the spiralling rays emerge, is of real gold. The dynamic character of the mosaic is apparent in the rays, which are reminiscent of the protuberances flung out by the real sun. Four vineleaves surround the circle, while in the center we see a Bacchant with wineskin and jar. The Dionysian traits of the picture are unmistakable." 


\section{Acknowledgment}

In his participation in some of the work reviewed here the author has been fortunate to be associated with Dr. Norman F. Ness of Goddard Space Flight Center and Dr. Robert Howard of the Mount Wilson and Palomar Observatories.

This research was supported in part by the Office of Naval Research under contract Nonr-3656(26), and by the National Aeronautics and Space Administration under grants NsG 243-62 and NGR 05-003-230. 


\section{References}

AHLUWALIA, H. S. and DESSLER, A. J.: 1962, Planetary Space Sci. 9, 195.

ALFVÉN, H. : 1956, Tellus 8, 1.

ALFVÉN, H.: 1966, Magnetism and the Cosmos, Oliver and Boyd, Edinburgh, p. 246.

ANDERSON, K. A. and LIN, R. P.: 1966, Phys. Rev. Letters 16, 1121.

ASBRIDGE, J. R., BAME, S. J., FELTHAUSER, H. E., and GOSLING, J. T.: 1967, Trans. Amer. Geophys. Union 48, 172.

BABCOCK, H. W.: 1953, Astrophys. J. 118, 387.

BARTELS, J.: 1932, Terrest. Magnetism Atmospheric Elec. 37, 1.

BARTELS, J.: 1963, Ann. Geophys. 19, 1.

BARTLEY, W. C., BUKATA, R. P., MeCRACKEN, K. G., and RAO, U. R.: 1966, J. Geophys. Res. 71, 3297.

BASLER, R. P.: 1966, Planet. Space Sci. 14, 1193.

BELL, B.: 1961, Smithsonian Contributions to Astrophysies 5, 69.

BIERMANN, I.: 1951, Z. Astrophys. 29, 274.

BILLINGS, D. E. and ROBERTS, W. 0.: 1964, Astrophys. Norweg. 9, 147.

BRANDT, J. C.: 1966, Astrophys. J. 144, 1221.

BRANDT, J. C.: 1967, Astrophys. J. 147, 201. 
BRYANT, D. A., CLINE, T. L., DESAI, U. D., and McDONAID, F. B.: 1965, Phys. Rev. Letters 14, 481.

BUMBA, V. and HOWARD, R.: 1965, Astrophys. J. 141, 1502.

BUMBA, V. and HOWARD, R.: 1966, Astrophys. J. I43, 592.

CHAPMAN, S.: 1954, Astrophys. J. 120, 151.

CHREE, C. and STAGG, J. M.: 1927, Phil. Trans. Roy. Soc. 227A, 21.

COLFMAN, P. J. JR., DAVIS, L. JR, SMITH, E. J., and JONES, D. E.: 1966, J. Geophys. Res. 71, 2831.

COLEMAN, P. J. JR., DAVIS, L. JR., SMITH, E. J., and JONES, D. E.: 1967, J. Geophys. Res. 72, 1637.

CORTIE, A. L.: 1913, Mon. Not. Roy. Astr. Soc. 73, 52.

DAVIS, L.: 1965, Stellar and Solar Magnetic Fields, R. Lulst, Ed., North-Holland Amsterdam.

DESSLER, A. J.: 1967, Revs. Geophys. 5, 1.

DICKE, R. H.: 1964, Nature 202, 432.

DICKE, R. H. and GOLDENBERG, H. M.: 1967, Phys. Rev. Letters 18, 313.

DODSON-PRINCE, H. and HEDEMAN, E. R.: 1967, "History and Morphology of Solar Activity - 1964-1965", IQSY/COSPAR Symposium, London.

FAIRFIELD, D. H. and NESS, N. F.: 1967, J. Geophys. Res. 72, 2379. 
FAN, C. Y., GLOECKLER, G., and SIMPSON, J. A.: 1966, Proceedings of the International Conference on Cosmic Rays, London.

FERRARO, V. C. A. and BHATIA, V. B.: 1967, Astrophys. J. 147, 220.

GOSIING, J. T., ASBRIDGE, J. R., BAME, S. J., HUNDHAUSEN, A. J., and STRONG,

I. B.: 1967, J. Geophys. Res. $72,3357$.

GUSS, D. E.: 1964, Phys. Rev. Letters 13, 363.

HERDEG, W.: 1962, The Sun in Art, Graphis Press, Zurich.

HOWARD, R.: 1959, Astrophys. J. 130, 193.

HUNDHAUSEN, A. J., ASBRIDGE, J. R., BAME, S. J., GILBERT, H. E. and STRONG,

I. B.: 1967a, J. Geophys. Res. $72,81$.

HUNDHAUSEN, A. J., BAME, S. J., and NESS, N. F. : 1967b, J. Geophys. Res. 72, 5265.

HUNDHAUSEN, A. J., ASBRIDGE, J. R., BAME, S. $J=$; and STRONG; $I=B=: 1967 c$; J. Geophys. Res. 72, 1979.

KIEPENHEUER, K. O.: 1953, The Sun, G. Kuiper, Ed., University of Chicago Press, Chicago.

KRIMIGIS, S. M., VAN ALLEN, J. A., and ARMSTRONG, T. P.: 1967, Phys. Rev. Letters 18, 1204.

KRIVSKY, L.: 1965, J. Atmos. Terr. Phys. 27, 1307.

IIN, R. P. and ANDERSON, K. A.: 1966, J. Geophys. Res. $71,4213$.

LUST, R.: 1965, Stellar and Solar Magnetic Fields, North-Holland, Amsterdam.

MACKIN, R. J. JR. and NEUGEBAUER, M.: 1966, The Solar Wind, Pergamon Press, New York. 
MAYAUD, P. N.: 1967, "Calcul Préliminaire d'indices $K_{m}$, $K_{n}$ et $K_{s}$ ou $a_{m}$, $a_{n}$ et $a_{s}$, mesures de l'activité magnétique à l'échelle mondiale et dans les hémisphères Nord et Sud", Institut de Physique du Globe, Universite de Paris, Note no. 23.

MEYER, J.: 1966, J. Geophys. Res. 71, 2397.

MICHEL, F. C.: 1964, J. Geophys. Res. 69, 4182.

MICHEL, F. C.: 1967, J. Geophys. Res. 72, 1917.

MODISETTE, J. L.: 1967, J. Geophys. Res. 72, 1521.

MORI, S., UENO, H., NAGASHIMA, K., and SAGISAKA, S.: 1964, Rept. Ionosphere Space Res. Japan 18, 275.

MUSTEL, E. R.: 1967, "Origin of the Solar Wind and its Astrophysical Aspects, IQSY/COSPAR Symposium, London.

MCCRACKEN, K. G. and NESS, N. F.: 1966, J. Geophys. Res, 71, 3315.

MCCRACKEN, K. G., RAO, U. R., and BUKATA, R. P.: 1966, Phys. Rev. Letters 17, 928.

MCCRACKEN, K. G., RAO, U. R. and BUKATA, R. P.: 1967, J. Geophys. Res. 72, 4293.

McINTOSH, D. H.: 1959, Phil. Trans. Roy. Soc. London 251A, 525.

MCPHERRON, R. I. and WARD, S. H.: 19 , J. Geophys. Res. 72, 393.

NESS, N. F.: 1965, J. Geophys. Res. 70, 2989.

NESS, N. F.: 1966, J. Geophys. Res. 71, 3319. 
NESS, N. F. and WILCOX, J. M.: 1966, Astrophys. J. 143, 23. NESS, N. F. and WIICOX, J. M.: 1967, Solar Phys. (to be published). NEUGEBAUER, M. and SNYDER, C. W.: 1966, J. Geophys. Res. 71, 4469.

NEUGEBAUER, M. and SNYDER, C. W.: 1967, J. Geophys. Res. 72, 1823.

NEWTON, H. W. and NUNN, M. I.: 1951, Monthly Not1ces Roy. Astron. Soc. 111, 413.

NISHIDA, A.: 1966, Rep. Ionos. Space Res. Japan 20, 36.

O'GALIAGHER, J. J. and SIMPSON, J. A.: 1966, Phys. Rev. Letters 16, 1212.

PAI, I. G., BRIDGE, H. S. and LYON, E. F.: 1967, Trans. Amer. Geophys. Union $48,176$.

PARKER, E. N.: 1958, Astrophys. J. 128, 664.

PARKER, E. N.: 1963, Interplanetary Dynamical Processes, Interscience Publishers, New York.

PNEUMAN, G. W.: 1966, Astrophys. J. 145, 800.

PRIESTER, W. and CATTANI, D.: 1962, J. Atmos. Sc1. 19, 121.

RAO, U. R., MCCRACKEN, K. G. and BUKATA, R. P.: 1967, J. Geophys. Res. 72, 4325.

ROOSEN, J.: 1966, Bull. Astr. Inst. Netherlands 18, 295.

ROTHWELL, P. and GREENE, C.: 1966, "Spatial and Temporal Distribution of Energetic Electrons in the Outer Radiation Belt", University of Southhampton preprint.

RYDER, P. and HATTON, C. J.: 1967, Proceedings of the International Conference on Cosmic Rays, Calgary. 


\section{7.}

SAKURAI, K.: 1966a, Publ. Astron. Soc. Japan 19, 350.

SAKURAI, K.: 1966b, Rept. Ionos. Space Res. Japan 20, 501.

SCHATTEN, K. H. and WILCOX, J. M.: 1967, J. Geophys. Res. 72, 5185.

SHAPIRO, R.: 1967, J. Geophys. Res. 72, 4945.

SIMON, G. W. and LEIGHTON, R. B.: 1964, Astrophys. J. 140, 1120.

STENFIO, J. O.: 1966, Observatory 86, 73.

STRONG, I. B., ASBRIDGE, J. R., BAME, S. J. and HUNDHAUSEN, A. J.: 1967, Trans. Amer. Geophys. Union 48, 191.

WARWICK, C. S.: 1965, Astrophys. J. 141, 500.

WEBER, E. J. and DAVIS, L: 1967, Astrophys. J.148, 217.

WILCOX, J. M.: 1965, Irish Astronomical J. 7, 82.

WILCOX,J. M. and NESS, N. F.: 1965, J. Geophys. Res. 70, 5793.

WILCOX, J. M. and NESS, N. F.: 1967, Solar Phys. 1, 437.

WILCOX, J. M., RITCHIE, A. D. and NESS, N. F.: 1966, Univ. Calif. Space Science Lab., Ser. 7, Issue 53.

WILCOX, J. M. and SCHATTEN, K. H.: 1967, Astrophys. J. 147, 364.

WILCOX, J. M., SCHATTEN, K. H. and NESS, N. F.: 1967, J. Geophys. Res. 72, 19.

WILLIAMS, D. J.: 1966, J. Geophys. Res. 71, 1815.

WOLFE, J. H., SILVA, R. W., McKIBBIN, D. D. and MASON, R. H.: 1966, J. Geophys. Res. $71,3329$. 
Figure Captions

Fig. 1. Distribution of the interplanetary magnetic field direction observed by IMP-1 in the plane of the ecliptic and normal to the ecliptic, averaged over 5.46-minute intervals. Both histograms show the field angular distribution per unit solid angle; the dashed circles would correspond to an isotropic distribution of the same number of vectors. The distribution is peaked in directions corresponding to the spiral streaming angle. The angular intervals in which the field is predominantly away from the sun and predominantly toward the sun are labeled positive and negative in this figure, and represented by plus and minus signs in Figure 3. The distribution normal to the ecliptic shows that the interplanetary field is predominantly parallel rather than perpendicular to the ecliptic (WILCOX and NESS, 1965).

Fig. 2. Same as Figure 1, but averaged over 3-hour intervals (WIICOX and NESS, 1965).

Fig. 3. The plus signs (away from the sun) and minus signs (toward the sun) at the circumference of the figure indicate the direction of the measured interplanetary magnetic field during successive 3-hour intervals. Parentheses around a plus or a minus sign indicate a time during which the field direction has moved beyond the 'allowed regions' shown in Figure 1 for a few hours in a smooth and continuous manner. The inner portion of the figure is a schematic representation of a sector structure of the interplanetary magnetic field that is suggested by these observations. The deviations about the average streaming angle that are actually present are not shown (WILCOX and NESS, 1965). 
Fig. 4. Interplanetary magnetic field data for January 21 and 22, 1964 observed by IMP-1. F is the magnitude of the field in gammas, and $\phi$ and $\theta$ are defined in Figure 1. The points are shown at 5.46-minute intervals. The range in $\phi$ labeled positive in Figure 1 is shown with a plus sign in this figure (field predominantly away from the sun), and the range in $\phi$ labeled negative in Figure $l$ is indicated with minus signs (field predominantly toward the sun). The interplanetary field was directed predominantly away from the sun during the entire time interval covered by this figure. At the bottom of the figure the field rms variations in gammas are shown for three mutually perpendicular axes (WILCOX and IESS, 1965).

Fig. 5. Interplanetary magnetic field data for January 7, 1964 observed by IMP-1. The ordinates are the same as in Figure 4. In most of this figure the interplanetary field is predominantly directed away from the sun. At 2220 UT a sector boundary rotates past the satellite and the field direction changes to being predominantly toward the sun (WILCOX and NESS, 1965).

Fig. 6. Contours of equal field occurrence probability for 8 IMP-2 interplanetary sectors, October 4, 1964-November 27, 1964. Data have been normalized so that 1 designates isotropic distribution. Vertical dashed lines indicate theoretical spiral angles of $135^{\circ}$ and $315^{\circ}$ (FAIRFIELD and NESS, 1967).

Fig. 7. The component $B_{w}$ in the direction of the ideal spiral field observed by Mariner 4. A point is plotted for each 3-hour period in which some data were obtained. The value plotted for $B_{w}$ is the center value of the $1-\gamma$ range that was recorded most frequently during the corresponding 3-hour period. A plasma velocity of $350 \mathrm{~km} / \mathrm{sec}$ was used in computing $\mathrm{B}_{\mathrm{w}}$ (COLEMAN et al., 1967). 
Fig. 8. Autocorrelation of the observed direction of the interplanetary magnetic field. The large positive peak at about 27 days' lag indicates that the interplanetary magnetic field structure corotates with the sun. The solid line is from the observations, and the dashed line is the autocorrelation of the idealized $2 / 7,2 / 7,2 / 7,1 / 7$ sector structure shown in the center of Figure 3 (WILCOX and NESS, 1965).

Fig. 9. Simultaneous measurements of the interplanetary magnetic field vector by Pioneer 6 and IMP-3 in solar ecliptic coordinates. Individual 5.46minute time averages of the IMP-3 data have been advanced by 57.5 minutes to compare remarkably well with the observations by Pioneer 6 . The predicted time advance is approximately 53 minutes. At this time the two satellites are separated by $1.3 \times 10^{6} \mathrm{~km}$ and on opposite sides of the earth. The favorable comparison supports the model of corotation of interplanetary magnetic field features associated with the solar rotation and radial plasma flow (NESS, 1966).

Fig. 10. Superposed epoch analysis of the magnitude of the interplanetary magnetic field as a function of position within the $2 / 7$ sectors shown in Figure 3 . The abscissa represents position within the sector, measured in days, as the sector sweeps past the earth. The ordinate is the average magnitude at the same relative position within the sectors. The results are shown separately for the four sectors with field away from the sun, for the three sectors with field toward the sun, and for all sectors (WILCOX and NESS, 1965).

Fig. 11. Superposed epoch analysis of the solar wind velocity as a function of position within the $2 / 7$ sectors (After WILCOX and NESS, 1965, but with revised MIT solar wind data, PAI et al., 1967).

Fig. 12. Superposed epoch analysis of the solar wind density as a function of position within the $2 / 7$ sectors (After WILCOX and NESS, 1965, but with revised MIT solar wind data, PAI et al., 1967). 
Fig. 13. Observed sector structure of the interplanetary magnetic field, overlayed on the daily geomagnetic character index $\mathrm{C9}$, as prepared by the Geophysikalisches Institut in Göttingen. Light shading indicates sectors with field predominantly away from the sun, and dark shading indicates sectors with field predominantly toward the sun. Diagonal bars indicates periods of mixed polarity. An assumed quasi-stationary structure during 1964 is indicated (modified from NESS and WILCOX, 1967).

Fig. 14. Autocorrelation of the direction of the interplanetary magnetic field observed by IMP-3 (NESS and WILCOX, 1967).

Fig. 15. Three-hour average values of plasma velocity $v$, proton density $n_{p}$, and magnetic-field strength $B$, versus time. The time base is chosen to show the 27-day-recurrence features associated with solar rotation (NEUGEBAUER and SNYDER, 1967).

Fig. 16. Frequency of occurrence of the 3-hour averages of the ratio of magnetic-energy density $B^{2} / 8 \pi$ to the positive-ion thermal-energy density $(3 / 2)$ $\left(n_{p} \mathrm{kT}_{\mathrm{p}}+n_{\mathrm{q}} \mathrm{kT}_{\mathrm{q}}\right)$. The shaded regions represent 3-hour periods for which a value of proton density could be calculated for fewer than half of the plasma spectrums (NEUGEBAUER and SNYDER, 1967).

Fig. 17. Frequency distribution of the 3-hour average values of the Alfvén velocity $v_{A}=H /\left[4 \pi m_{p}\left(n_{p}+4 n a\right)\right]^{1 / 2}$. The shaded regions represent 3-hour periods for which a value of proton density could be calculated for fewer than half of the plasma spectrums (NEUGEBAUER and SNYDER, 1967).

Fig. 18. A contour mapping in the plane of observation of a typical proton velocity distribution function derived from Vela 3 data. The small triangle 
indicates the mean velocity. $\mathrm{B}_{\mathrm{p}}$ indicates the 5.46-minute average magnetic field, projected onto the plane of observation, for the time interval centered at 0448 UT (HUNDHAUSEN et al., 1967b).

Fig. 19. The magnetic field direction $\phi_{b}$ and direction of maximum proton temperature $\phi_{a}$, plus $180^{\circ}$ (measured on Vela 3B), for a 6-hour period on August 4, 1965 (HUNDHAUSEN et al., 1967b).

Fig. 20. A scatter plot of magnetic field direction $\phi_{b}$ and direction of maximum proton temperature $\phi_{a}$ for the period July 24-August 26, 1965 (HUNDHAUSEN et al., 1967b).

Fig. 21. The magnetic field and cosmic-ray azimuths during the interval 1600 to 2000 UT, December 30, 1965. Note the very close correspondence of the two azimuths during the abrupt change in direction around 1800 UT (McCRACKEN and NESS, 1966).

Fig. 22. Two directional counting rates and the azimuth of the cosmic-ray anisotropy $\left(\theta_{A}\right)$ during the initial stages of the flare effect that commenced on December 30, 1965. The data are 7.5-minute samples (BARTLEY et al., 1966).

Fig. 23. Solar wind velocity and proton temperature on July 29-30, 1964 observed by Vela 2 (HUNDHAUSEN et al., 1967c).

Fig. 24. Counting rate of the University of Chicago experiment on IMP-1 detecting protons having an energy of a few Mev. Three solar rotations are shown. The recurring stream of protons is for the most part contained within the positive sector. The cross hatching indicates the uncertainty in the position of the sector boundary caused by the position of the satellite near perigee (WILCOX and NESS, 1965). 
Fig. 25. Integral proton intensities for solar rotations Nos. 1773 to 1778. Each datum point represents a six-hour average and, unless otherwise shown, errors are smaller than the data symbols. Only data taken above $73000 \mathrm{~km}$ are used. The arrows indicate sudden commencements followed by magnetic storms, and the dashed line is a 27-day fiducial marker. Three flare-associated events occur in late April and mid-June (BRYANT et al., 1965).

Fig. 26. Integral energy spectra of the observed proton intensity increases. Data are shown for each event when the $>6-\mathrm{Mev}$ component was at maximum intensity (BRYANT et al., 1965).

Fig. 27. Differential energy spectrum of protons from the University of Chicago pulse height analyser on IMP-I (FAN et al., 1966).

Fig. 28. Superposed epoch analysis of the Deep River neutron monitor counting rate as a function of position within the $2 / 7$ sectors (WIICOX and NESS, 1965).

Fig. 29. Heliocentric pattern of the additional anisotropic flows of cosmic rays $(\triangle D S)$, the corresponding solar coronal green line indices $G_{6}$ and the disturbances of $\Sigma K_{p}$ and $\Delta I$ of cosmic rays on the earth (MORI et al., 1964).

Fig. 30. Cosmic ray (Deep River neutron monitor) variations during an average eight-day sector shown in Figure 3. a), The mean diurnal variation during the central four days. b) - e), The mean diurnal variation during the central four days subtracted from the mean diumal variation during $b$ ), days 1 and 2 ; $c$ ), days 3 and 4 ; d) days 5 and 6 ; and e), days 7 and $8 . f$ ) - g), The difference in mean diurnal variation between days 1 and 2 and days 7 and 8 is shown in $f$ ), for all sectors; $g$ ), for away sectors; and $h$ ), for toward sectors (RYDER and HATTON, 1967). 
Fig. 31. The streaming of cosmic rays postulated to explain the variation of the anisotropy through a sector (RYDER and HATTON, 1967).

Fig. 32. Average interplanetary and magnetosheath field magnitude plotted at the average $\phi$ angle for each of the IMP-2 sectors. Magnetosheath fields are shown at the shock front for convenience although they represent measurements obtained throughout the magnetosheath. Average solar ecliptic $\theta$ angles are all less than $16^{\circ}$ (FAIRFIELD and NESS, 1967).

Fig. 33. Simultaneous observations of directional intensities of solar protons with Explorer 33 and Injun IV. The smooth curve is drawn through halfhour-averaged counting rates of Explorer 33. Each plotted point represents a polar-cap averaged counting rate for Injun IV. The respective sets of data are superimposed on the same absolute intensity basis (to within 25\%) by displacing the counting-rate scale of Injun IV data upward by one decade (KRIMIGIS et al., 1967).

Fig. 34. Superposed epoch analysis of the geomagnetic activity index 24-hour sum $K_{p}$ as a function of position within the $2 / 7$ sectors (WILCOX and NESS, 1965 ). Fig. 35. Planetary magnetic 3-hour range indices $K_{p}$. The sector boundaries are indicated with vertical lines. The cross-hatched areas indicate uncertainties in the position of the sector boundaries caused by the position of the satellite near perigee. An approximation to the average shape shown in Figure 34 can be seen in each individual sector (WILCOX and NESS, 1965).

Fig. 36. Intensity contour map of the outer radiation zone near the magnetic equatorial plane, plotted as a function of $\mathrm{L}$ and time for the month of January 1965. The intensity contours are plotted in flux units of $10^{3}$ electrons $\mathrm{cm}^{-2} \mathrm{sec}^{-1}$. 
The polarity of the interplanetary magnetic field (measured from Mariner 4) is indicated at the top of the map and the $K_{p}$ indices are shown at the bottom (ROTHWELL and GREENE, 1966).

Fig. 37. Trapped electron intensities for the four consecutive solar rotations beginning October 6, 1963. Data are for electron energies $\geqslant 1.2 \mathrm{Mev}, \mathrm{L}=4.5$ and $1100 \mathrm{~km}$. The occurrence of the sector boundaries is shown, and $K_{p}$ is plotted at the bottom of each graph (WILLIAMS, 1966).

Fig. 38. Smoothed probability of pearl occurrence as a function of both day in solar sector and local time (McPHERRON and WARD, 1967).

Fig. 39. Ring current intensity, represented roughly by the daily mean horizontal intensity at Honolulu, during the three month period for which TMP-l sector data are available. Arrows indicate the passage of the sector boundary. In three sectors, whose onset is marked as c, solar protons of a few Mev are observed at the satellite (NISHIDA, 1966).

Fig. 40. Average response of the geomagnetic activity index (24-hour sum $K_{p}$ ) as a function of position within a sector for the IMP-1 and IMP-2 satellites (NESS and WILCOX, 1967).

Fig. 41. Average value of $K_{p}$ for the away sectors and the toward sectors within each Bartels solar rotation observed by IMP-3 in 1965 (SCHATTEN and WIICOX, 1967).

Fig. 42. Variation with universal time of Mayaud's indices of geomagnetic activity (see text) at the solstices and at the equinoxes. The crosses represent the $a_{p}$ index. Sample A includes all three-hourly intervals, and sample B includes three-hourly intervals with $K_{p} \geqslant 20$ (MAYAUD, 1967). 
Fig. 43. Seasonal variations of Mayaud's indices of geomagnetic activity for the northern hemisphere and for the southern hemisphere. Sample A includes all three-hourly intervals, sample B includes three-hourly intervals with $K_{p} \geqslant 2_{0}$, and sample $c$ includes three-hourly intervals with $K_{p} \geqslant 4+$ (MAYAUD, 1967).

Fig. 44. Harmonic dial for the 6-month wave in the equivalent recurrence numbers, averaged over 28 years before and including sunspot minima (Min), around sunspot maxima (Max), and for all years (All). The circles about the endpoints of the vectors denote the probable errors. Marked as radii are the equinoxes and the times when the sun's axis is most inclined toward the earth (MEYER, 1966).

Fig. 45. The semi-annual variation of the average $A_{p}$. Thirty-days-running means of all A $1932-1961$ (dots), Ap during M-region disturbances (crosses), absolute solar declination (full curve) and absolute heliographic latitude of the Earth (broken curve) (ROOSEN, 1966).

Fig. 46. Variation of $K_{p}$. To find the equivalent equinoctial $K_{p}$ from a given $K_{p}$ at a given date, say $7^{\circ}$ at 20 January, draw a curve parallel to the given curves from this point to the vertical corresponding to the equinoxes, and read the value of $K_{p}$ there. In the example the result is $8+($ ROOSEN, 1966).

Fig. 47. Crosscorrelation as a function of time lag between $\mathrm{K}_{\mathrm{p}}$ and the interplanetary field magnitude observed by IMP-I (WILCOX et al., 1967).

Fig. 48. Synoptic chart of photospheric magnetic fields observed with the solar magnetograph at Mt. Wilson Observatory for central meridian dates November 25, 1963 to December 11, 1963. This is a rectangular equal area projection. Solid lines represent positive polarity; dashed lines, negative polarity. 
Isogauss lines are for $2,4,8,12$, and 25 gauss. The equator is drawn across the center. The extrapolated position of the positive sector is indicated as described in the text (WIICOX and NESS, 1965).

Fig. 49. Crosscorrelation of nearby interplanetary magnetic field direction and photospheric magnetic field direction for solar latitudes from $25^{\circ} \mathrm{N}$ to the center of the visible disk (NESS and WILCOX, 1966).

Fig. 50. Autocorrelation of the photospheric magnetic field direction for latitudes at the center of the visible disk and $25^{\circ} \mathrm{N}$ thereof. The increase in period caused by differential rotation is quite apparent (NESS and wILCOX, 1966).

Fig. 51. Rotation period of the photospheric magnetic field as a function of latitude, as determined from autocorrelation analysis as shown in Figure 50. The solid line is the rotation period determined by Newton and Nunn from analysis of long-lived sunspots. The errows at left indicate the recurrence period of nearby interplanetary field and the uncertainty thereof (NESS and WILCOX, 1966).

Fig. 52. Autocorrelation of the photospheric magnetic field direction for heliographic latitudes from $35^{\circ} \mathrm{N}$ to $35^{\circ} \mathrm{S}$ in intervals of $5^{\circ}$. Seven solar rotations centered on the three rotations observed by IMP-I are included. The format is explained in the text (WILCOX and NESS, 1967).

Fig. 53. Superposed-epoch analysis of calcium plage structure obtained from the daily Fraunhofer Institute maps of the sun. The large sectors shown in Figure 3 are approximately centered at central meridian, so that the leading edge of the sector is at about $50^{\circ} \mathrm{W}$ and the trailing edge of the sector is about $50^{\circ} \mathrm{E}$ longitude (WILCOX and NESS, 1967). 
Fig. 54. Left: the daily geomagnetic character figure $C 9$ as published by the Geophysical Institute, Gottingen. Right: to the same scale, diagram showing an estimate of the central meridian passage of the preceding portions of unipolar magnetic regions (BUMBA and HOWARD, 1966).

Fig. 55. Recurrences of positive and negative pulses of geomagnetic activity (see text) (CHREE and STAGG, 1927).

Fig. 56. Plausible magnetic field pattern and isothermal surfaces near a sector boundary (modified from BIILINGS and ROBERTS, 1964).

Fig. 57. Schematic of structure within the solar wind illustrating filamentary structure within the interplanetary magnetic field. The field lines passing through a given filament tube lead to the site of the origin of the filament (MICHEL, 1967).

Fig. 58. A superposed epoch diagram of solar radio flux at $2800 \mathrm{Mc} / \mathrm{s}$, with zero day corresponding to large absorption of cosmic radio noise (BASLER, 1966 ).

Fig. 59. Same as Figure 58, except that zero day corresponds to low absorption of cosmic radio noise (BASLFR, 1966).

Fig. 60. Roman mosaic in Egypt (HERDEG, 1962). 


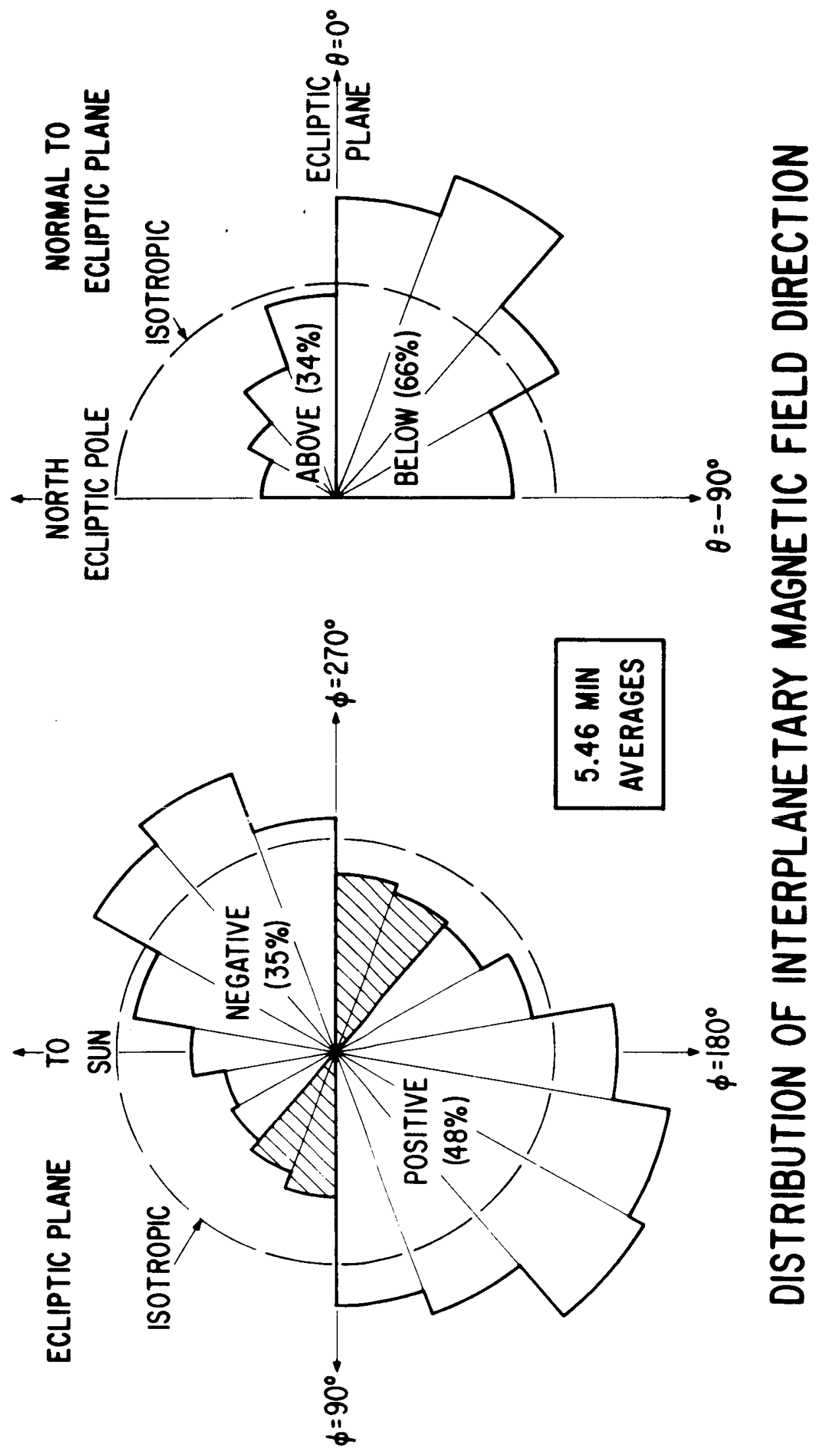

Figure 1 


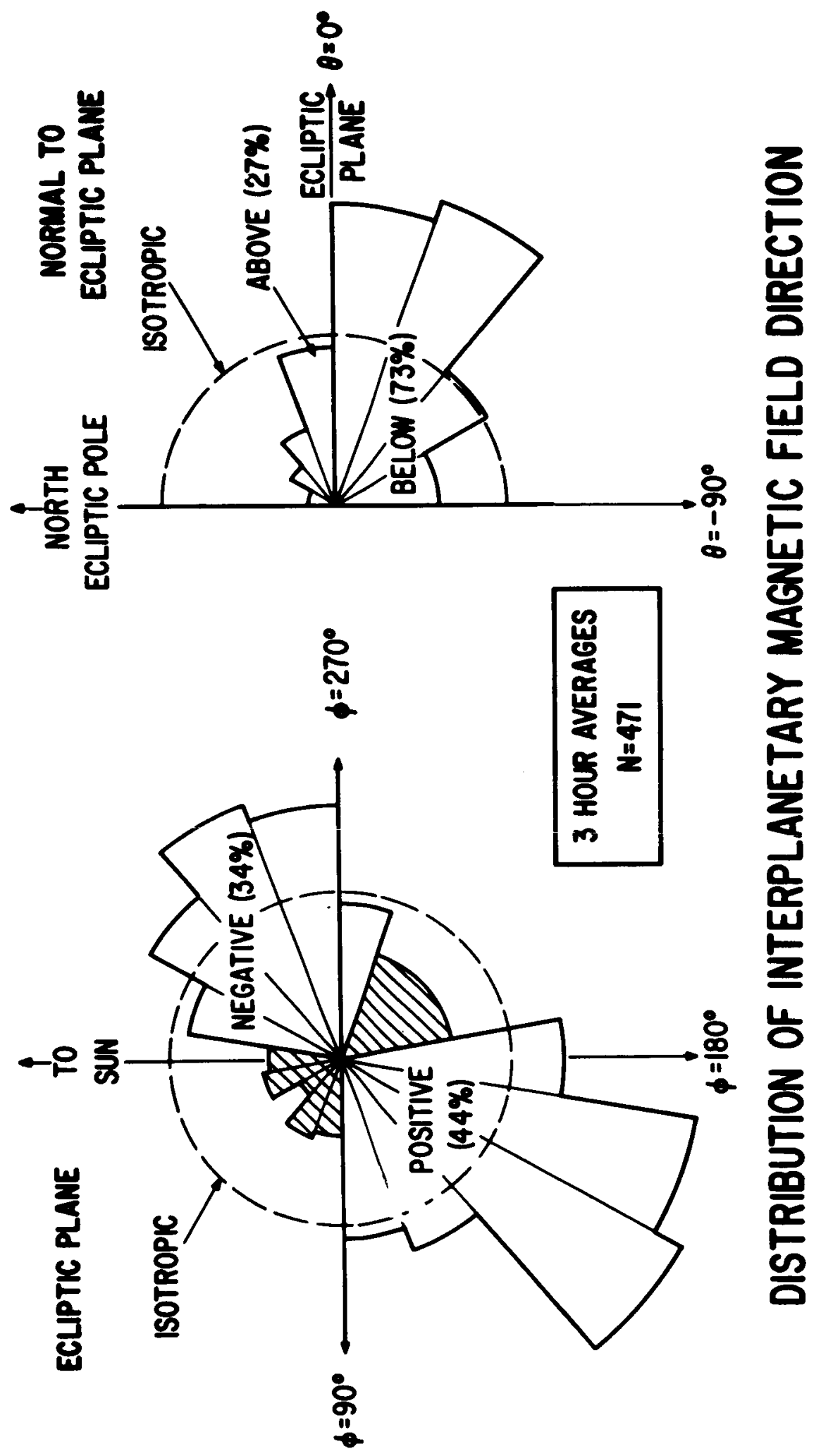

Figure 2 


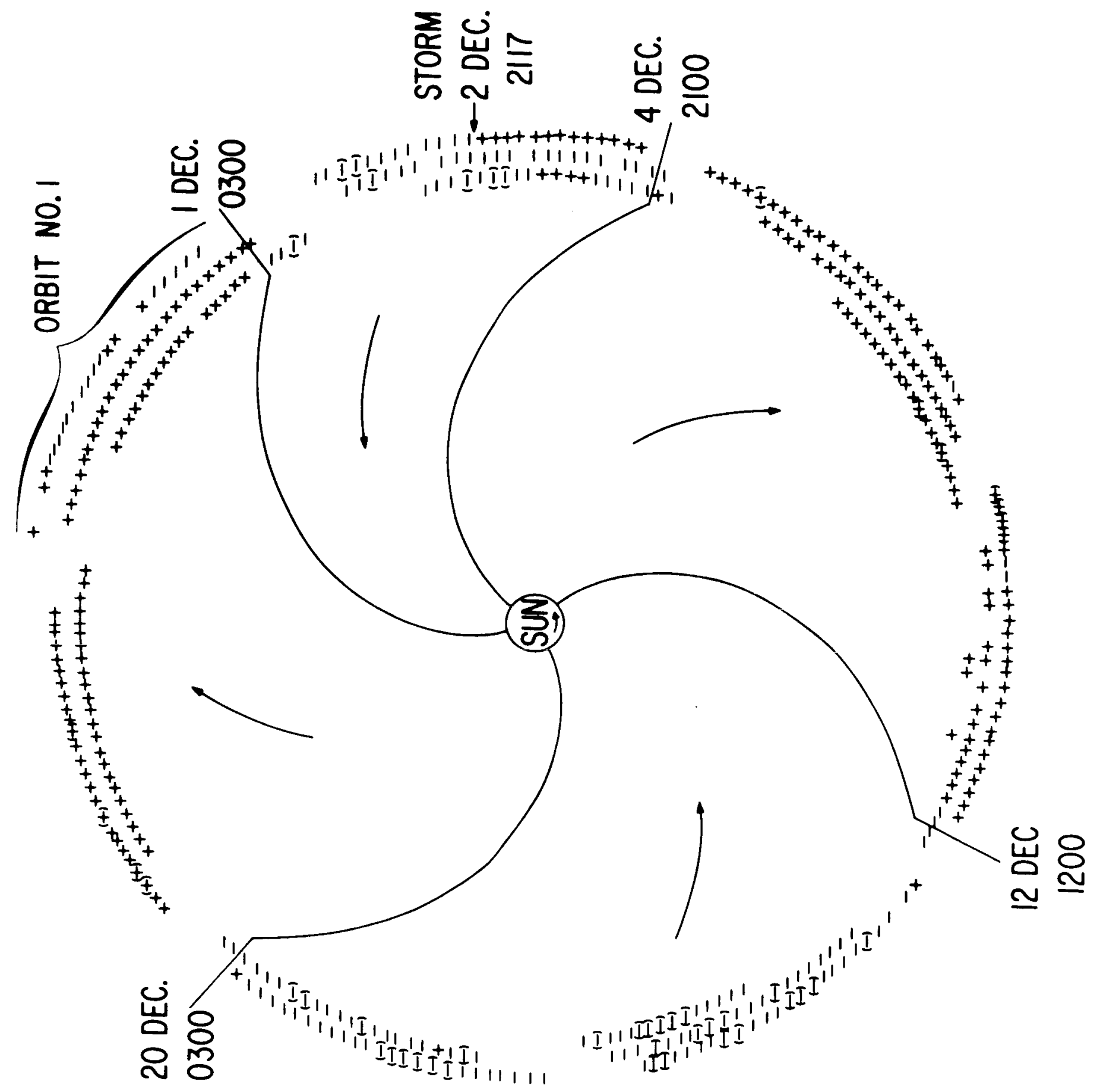

Figure 3 


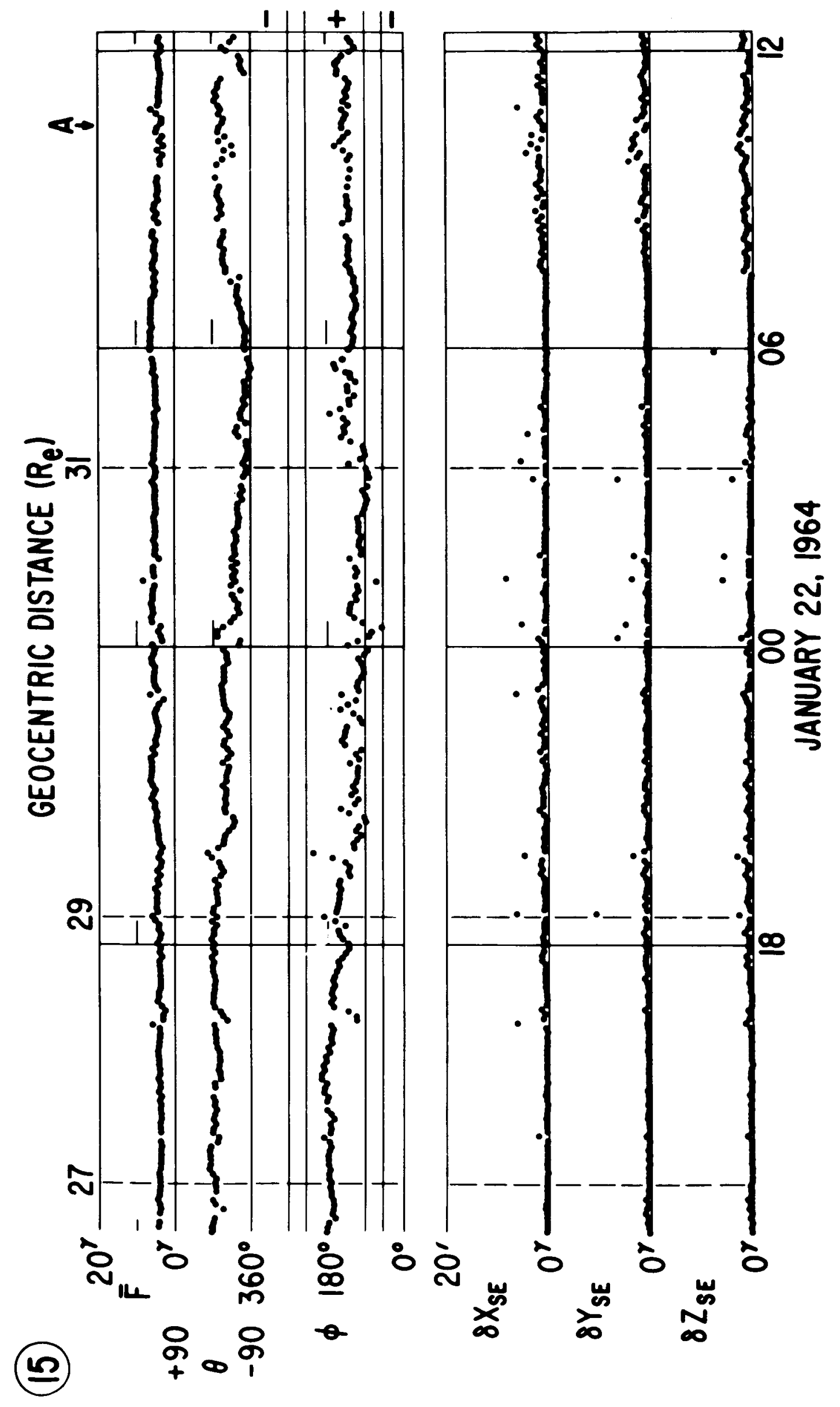

Figure 4 


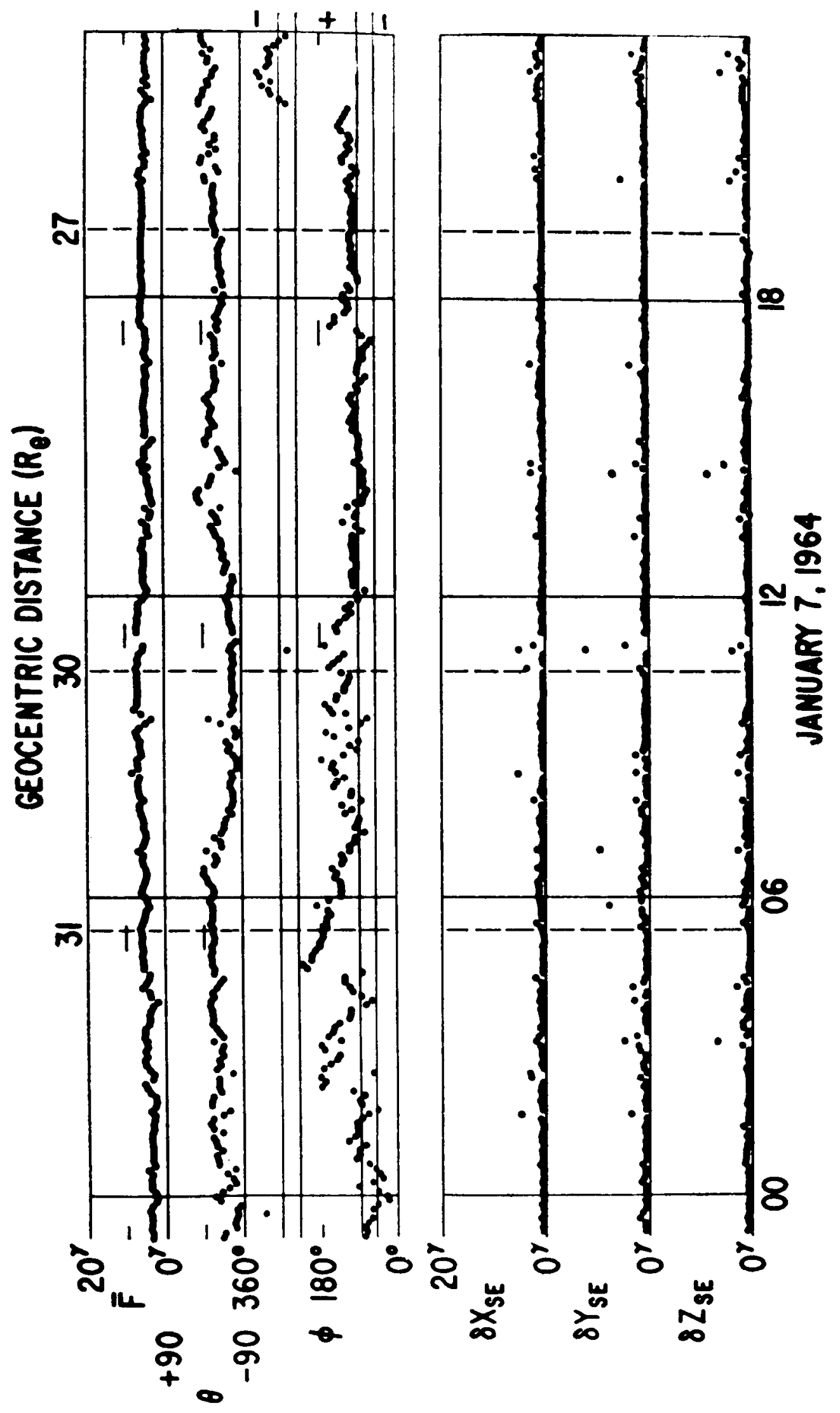

Figure 5 

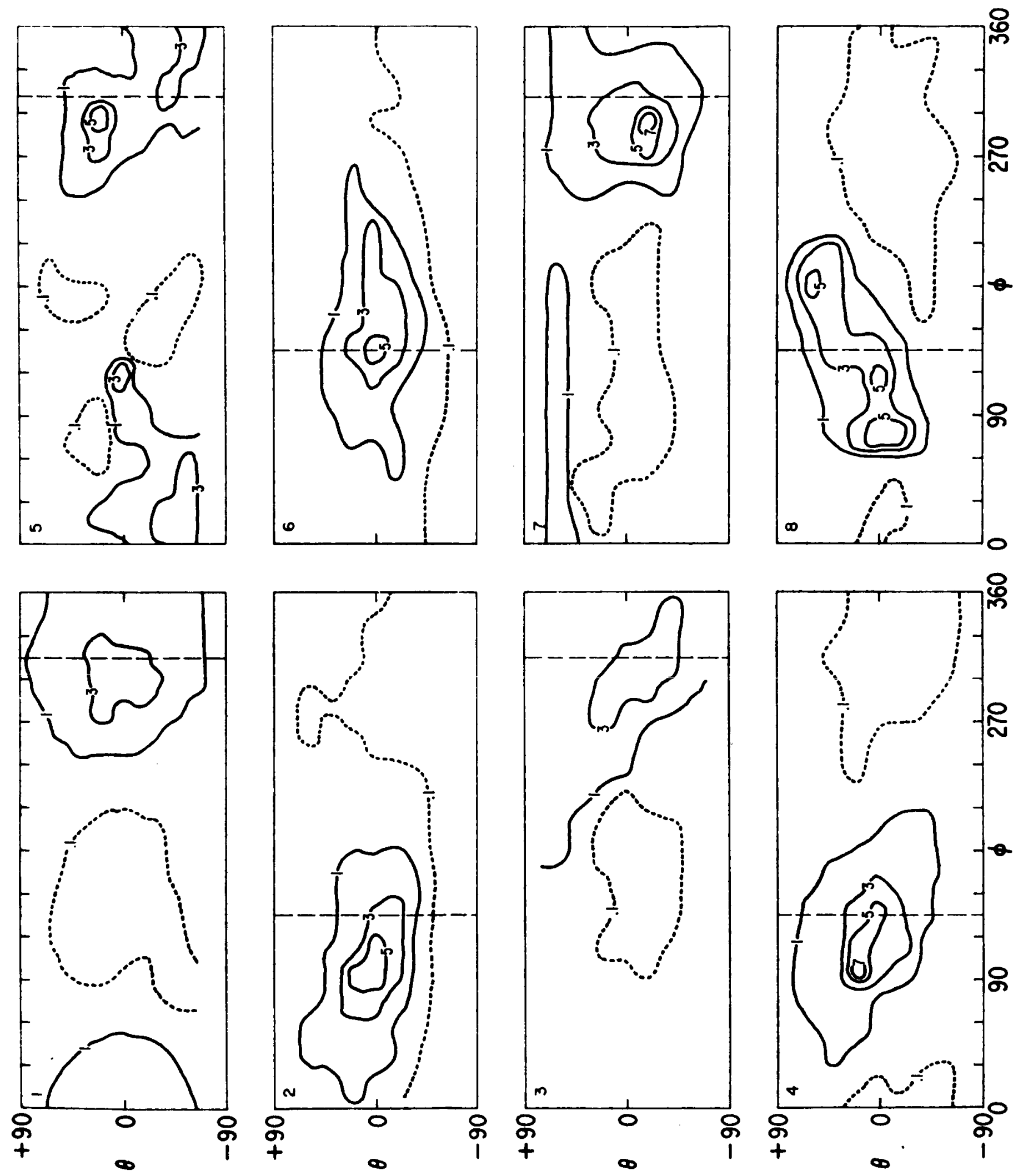


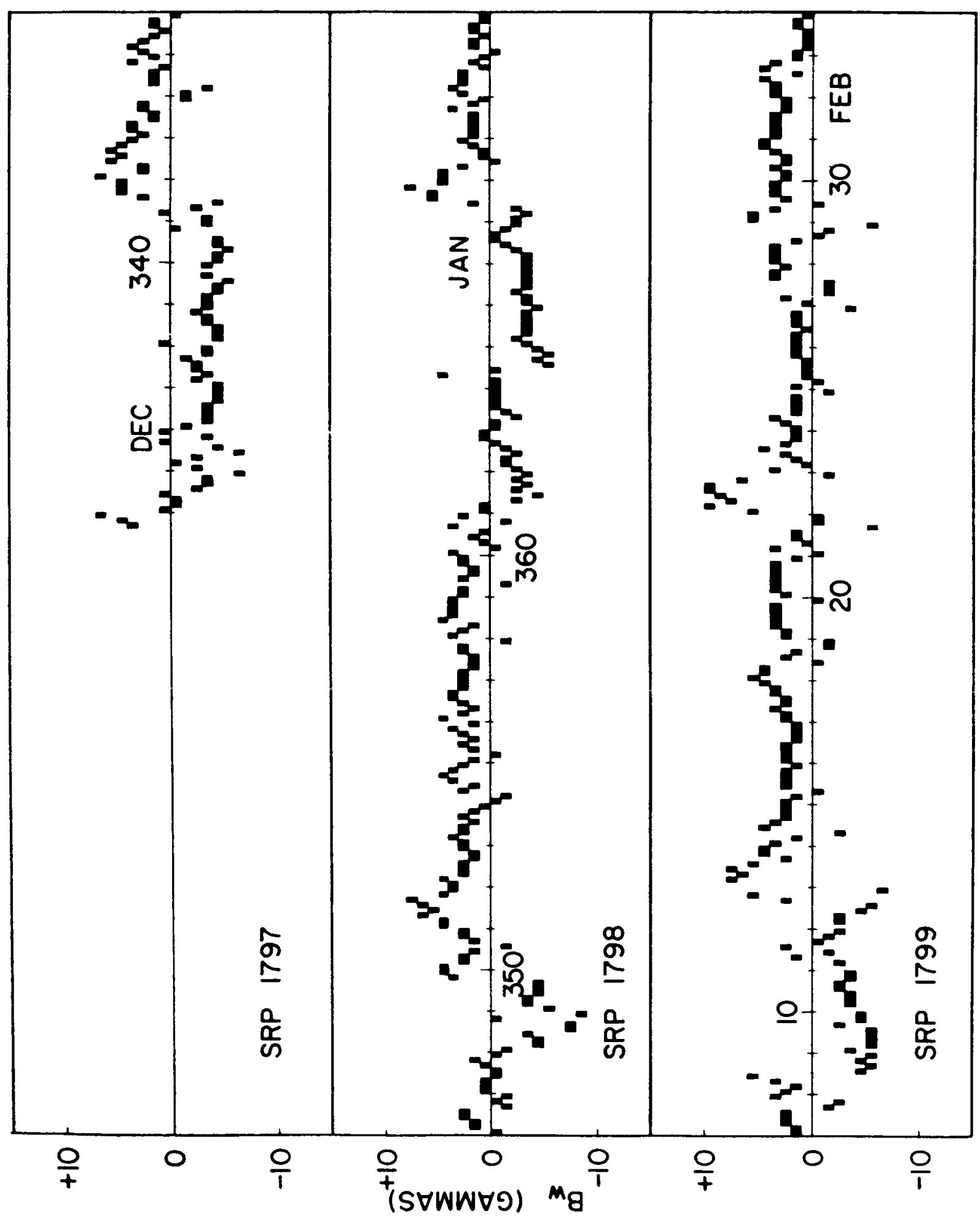

Figure 7 


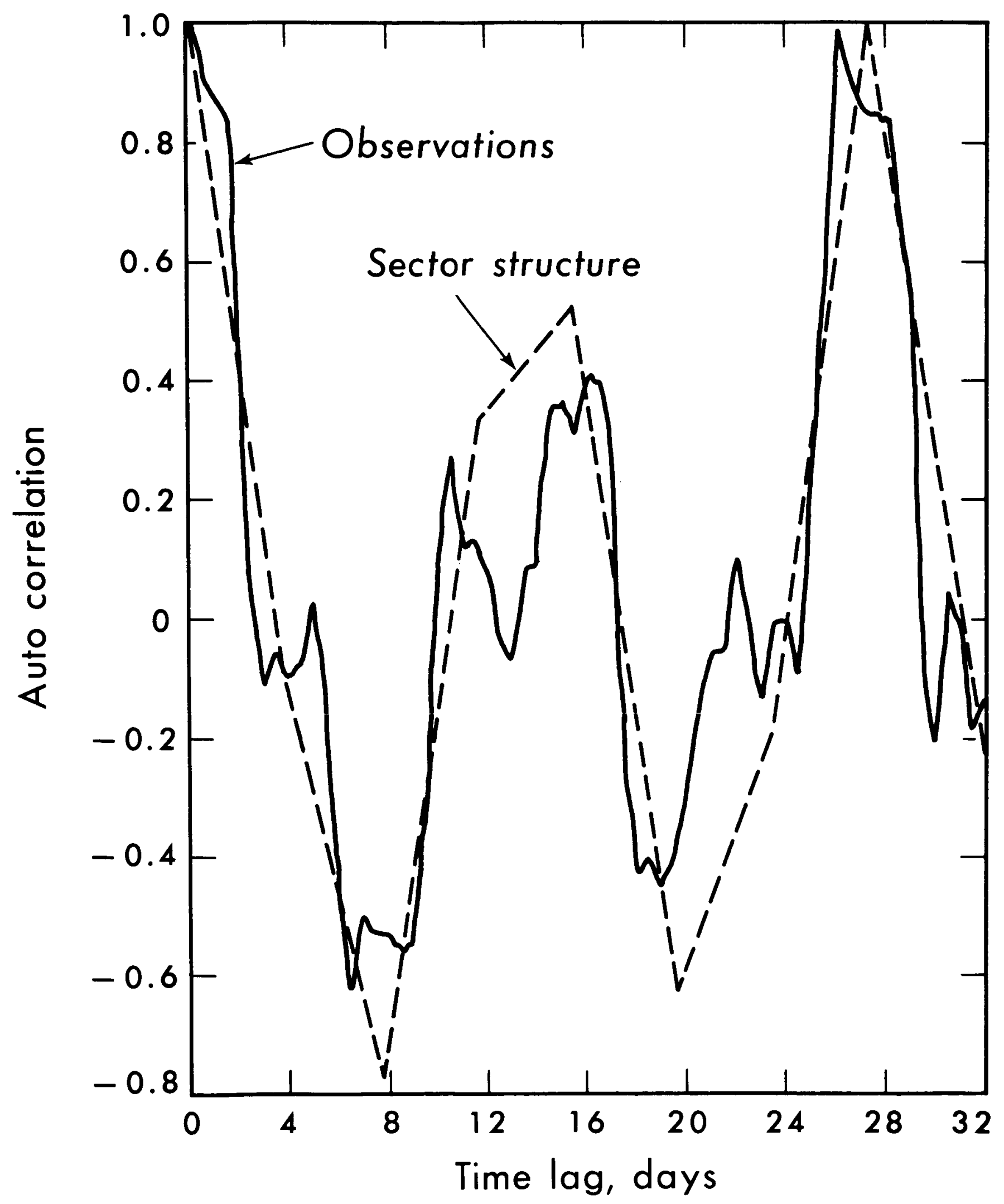

Figure 8 


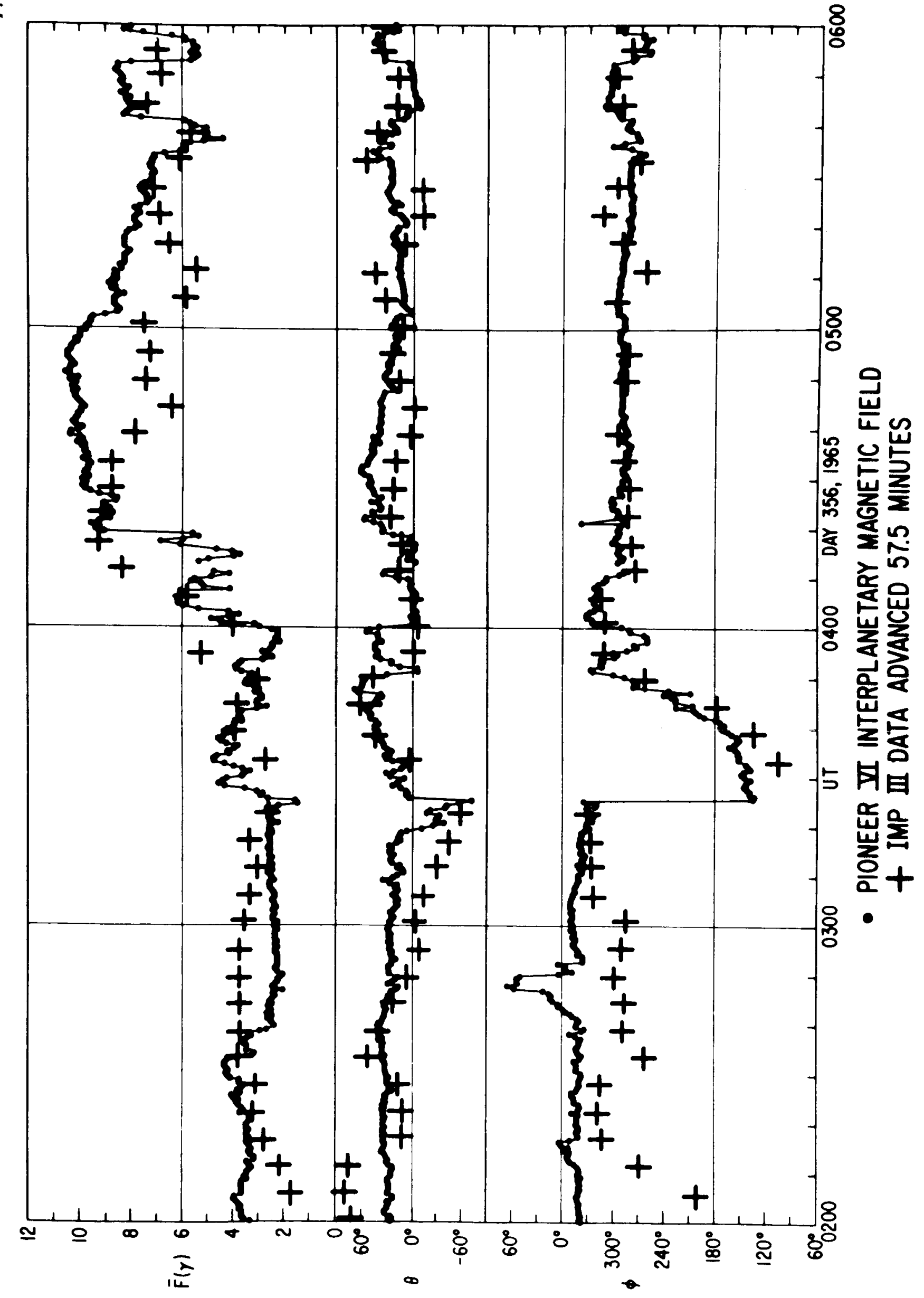

Figure 9 


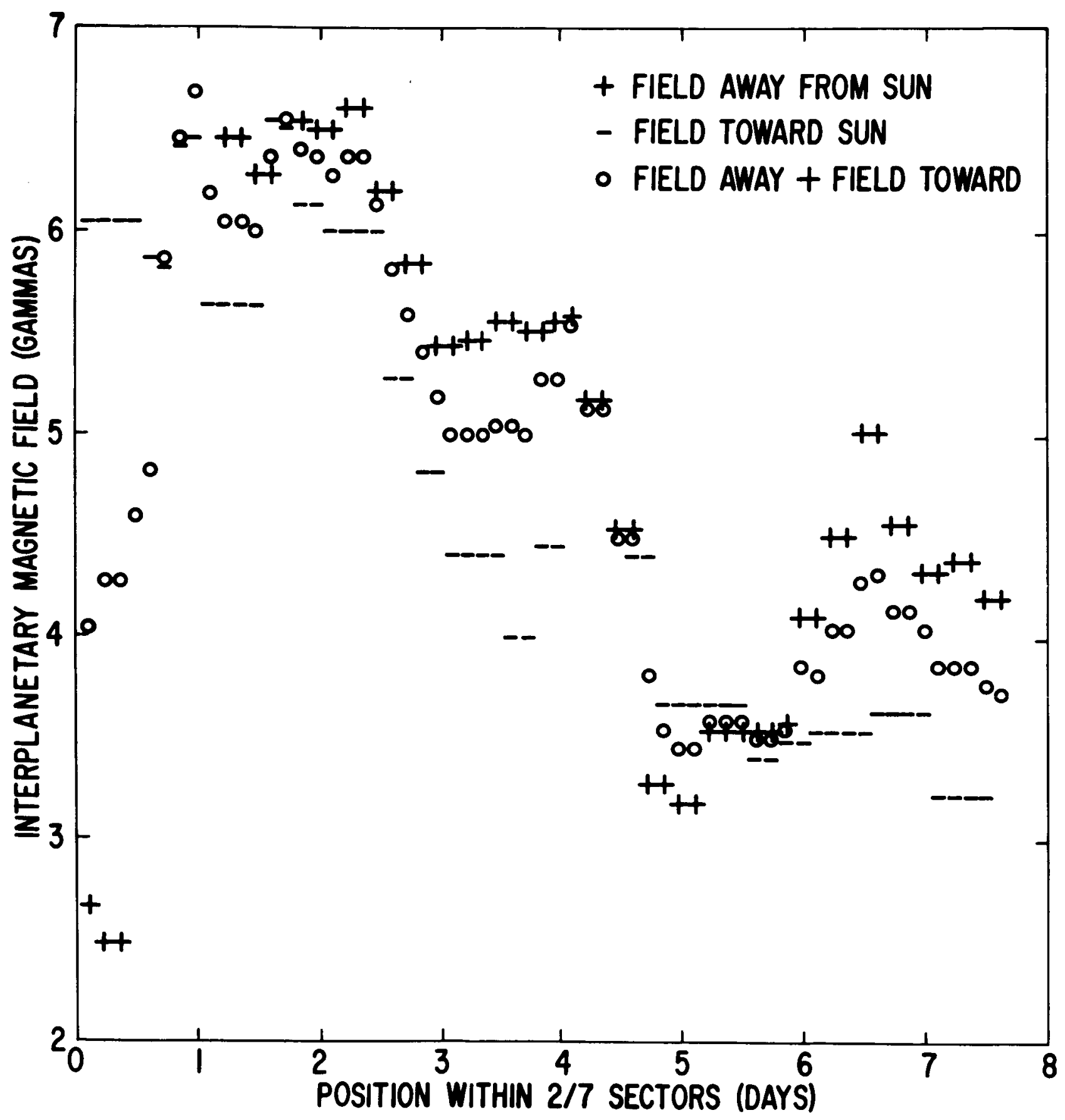




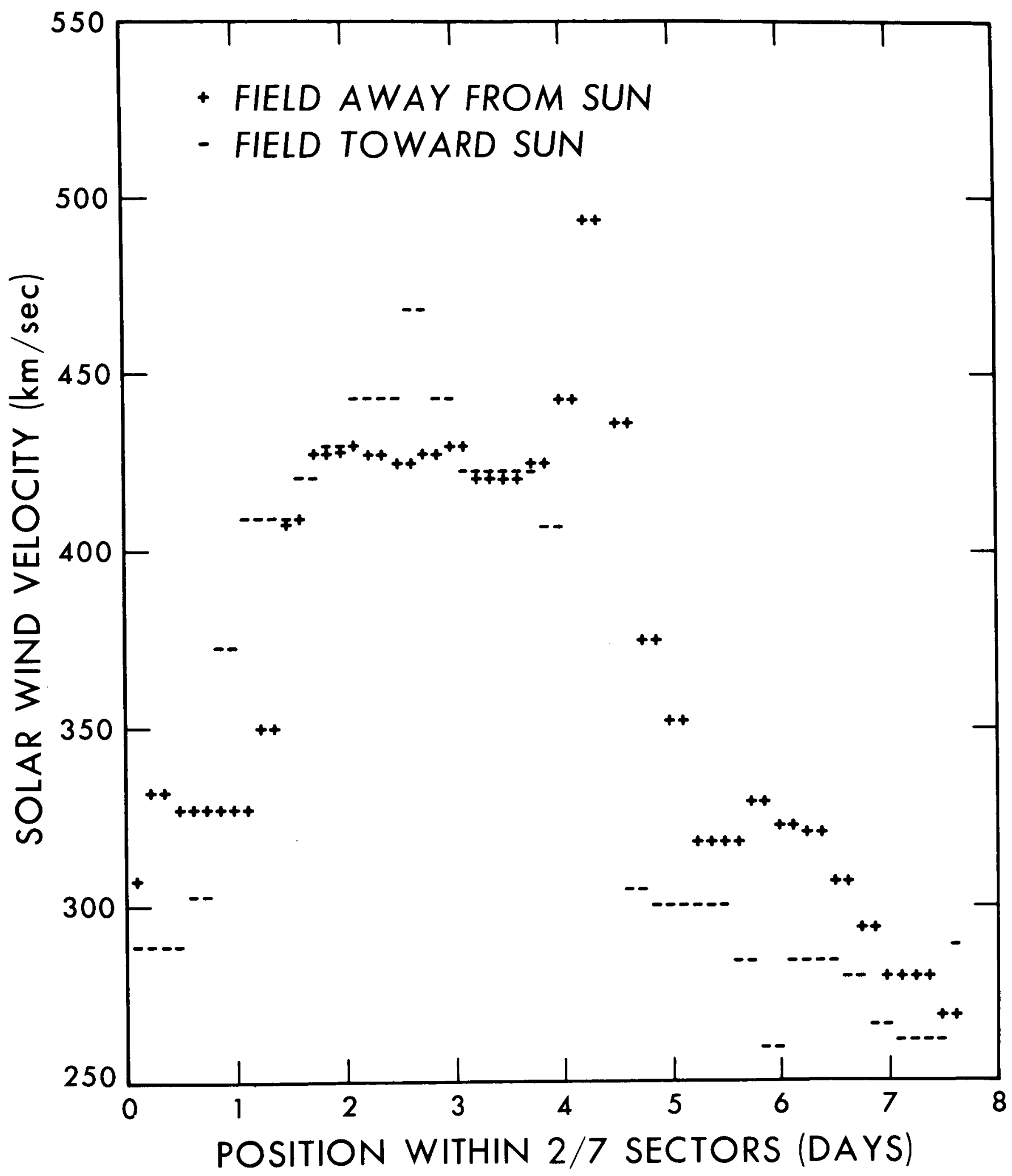




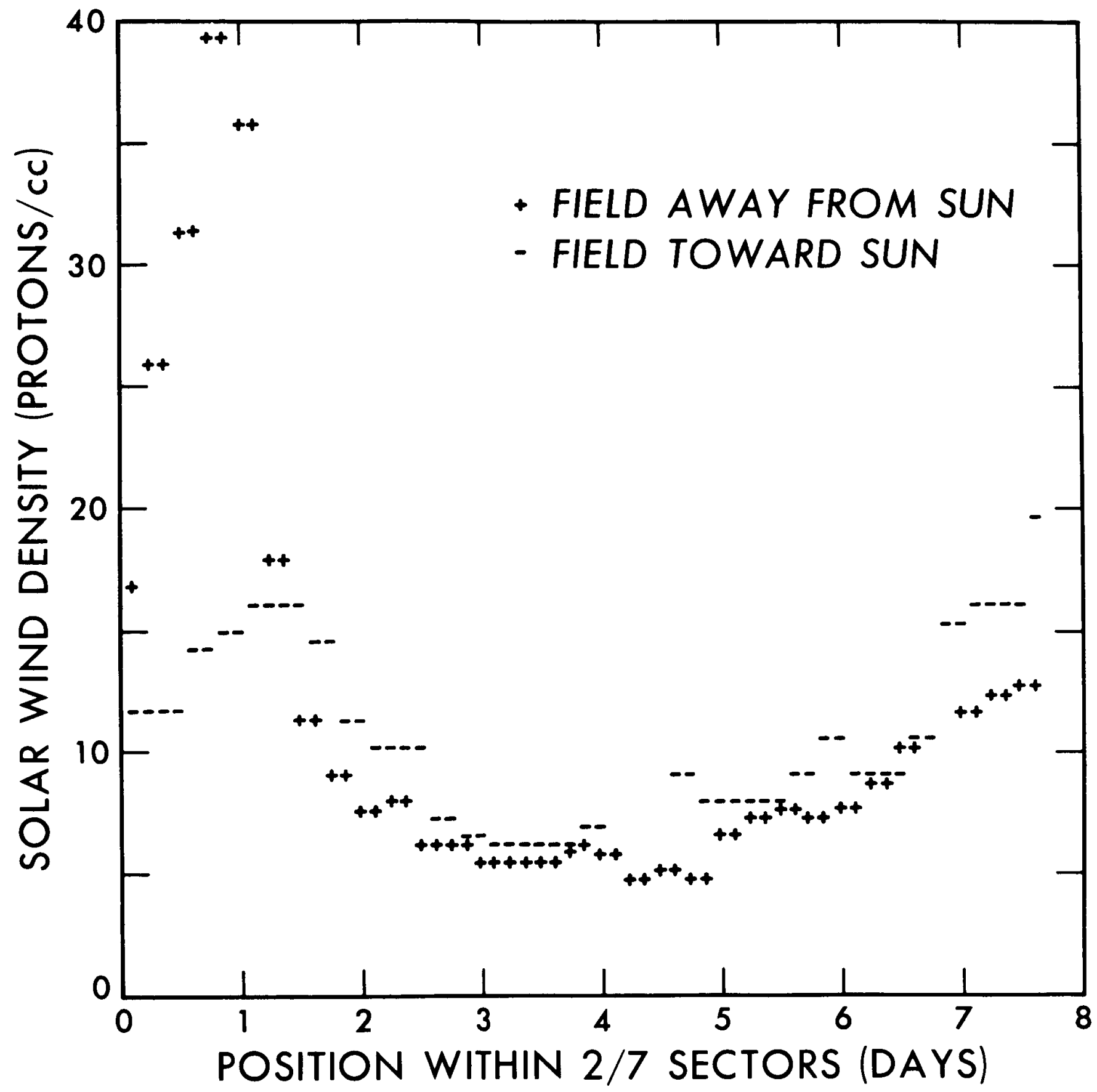




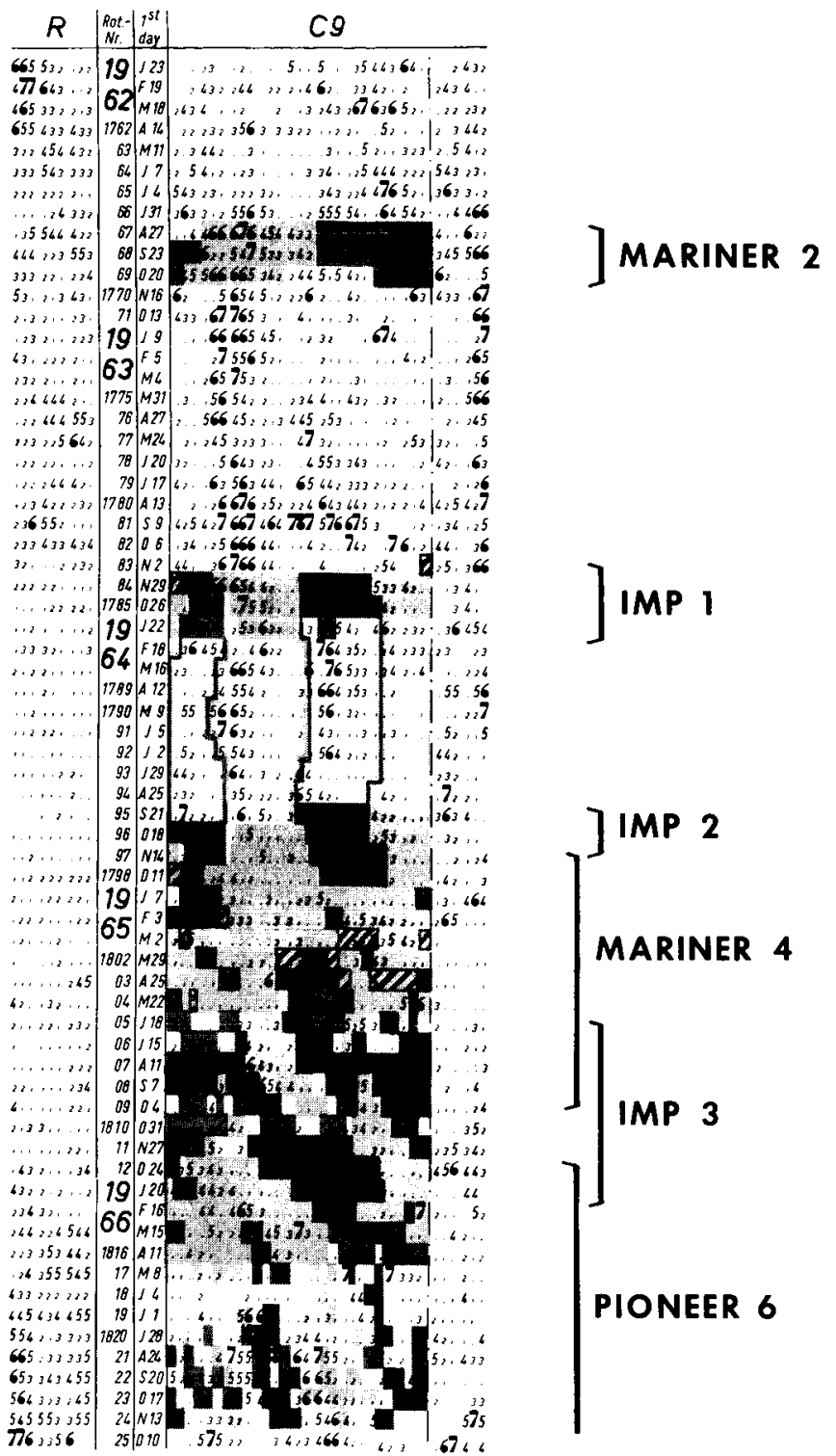

Figure 13 


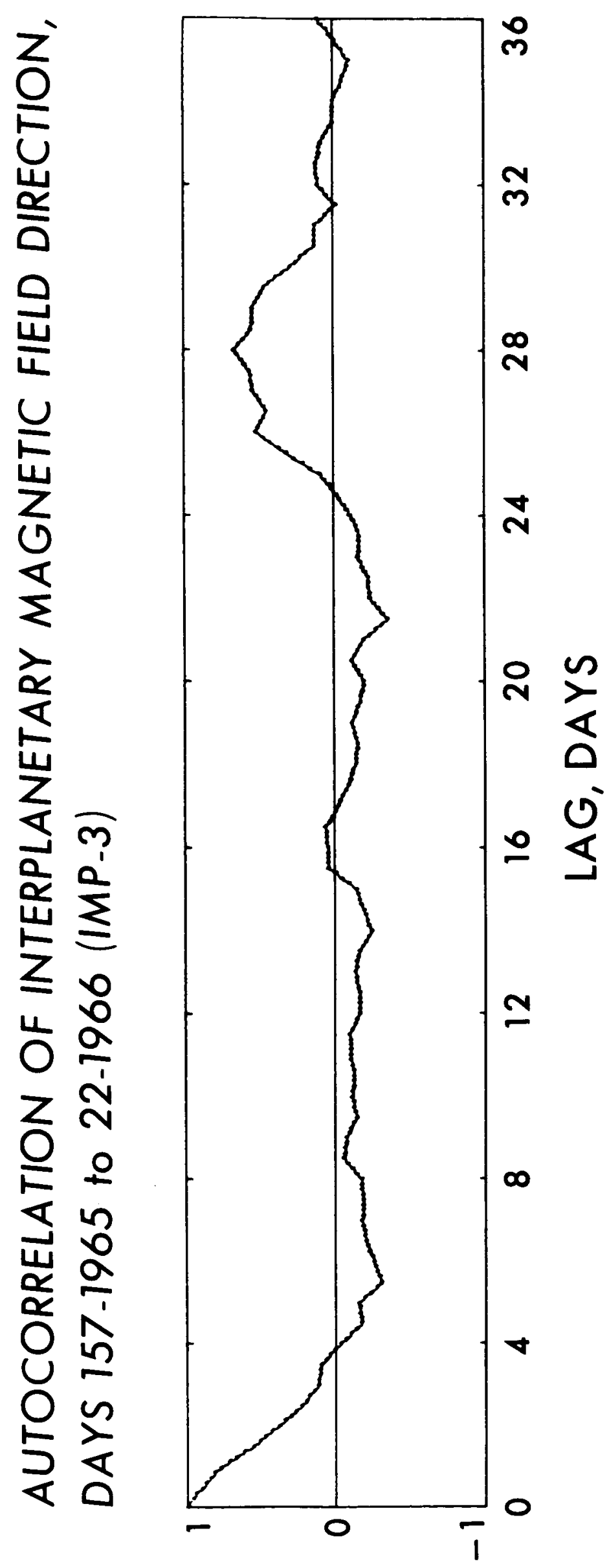

Figure 14 

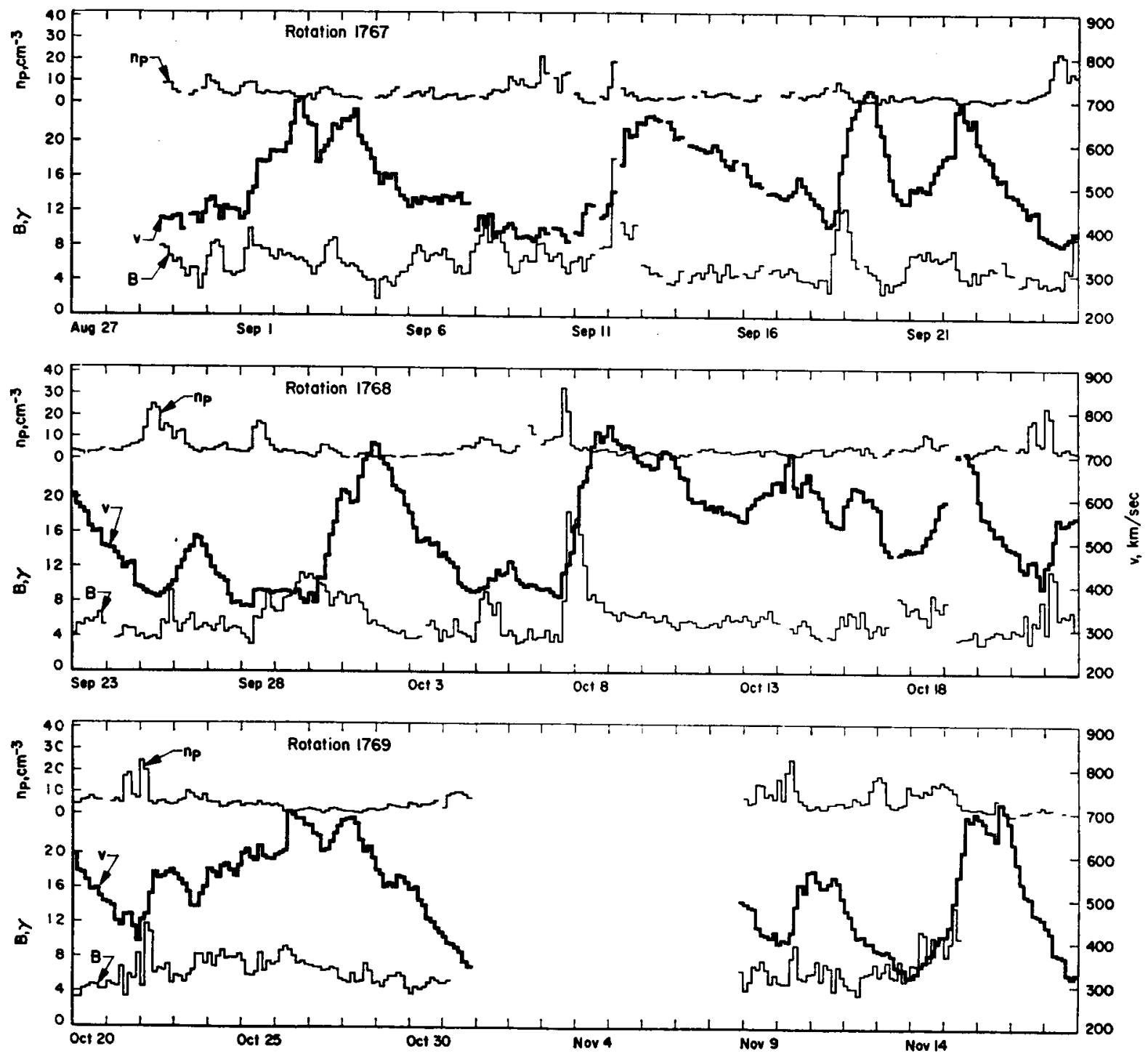

Figure 15 


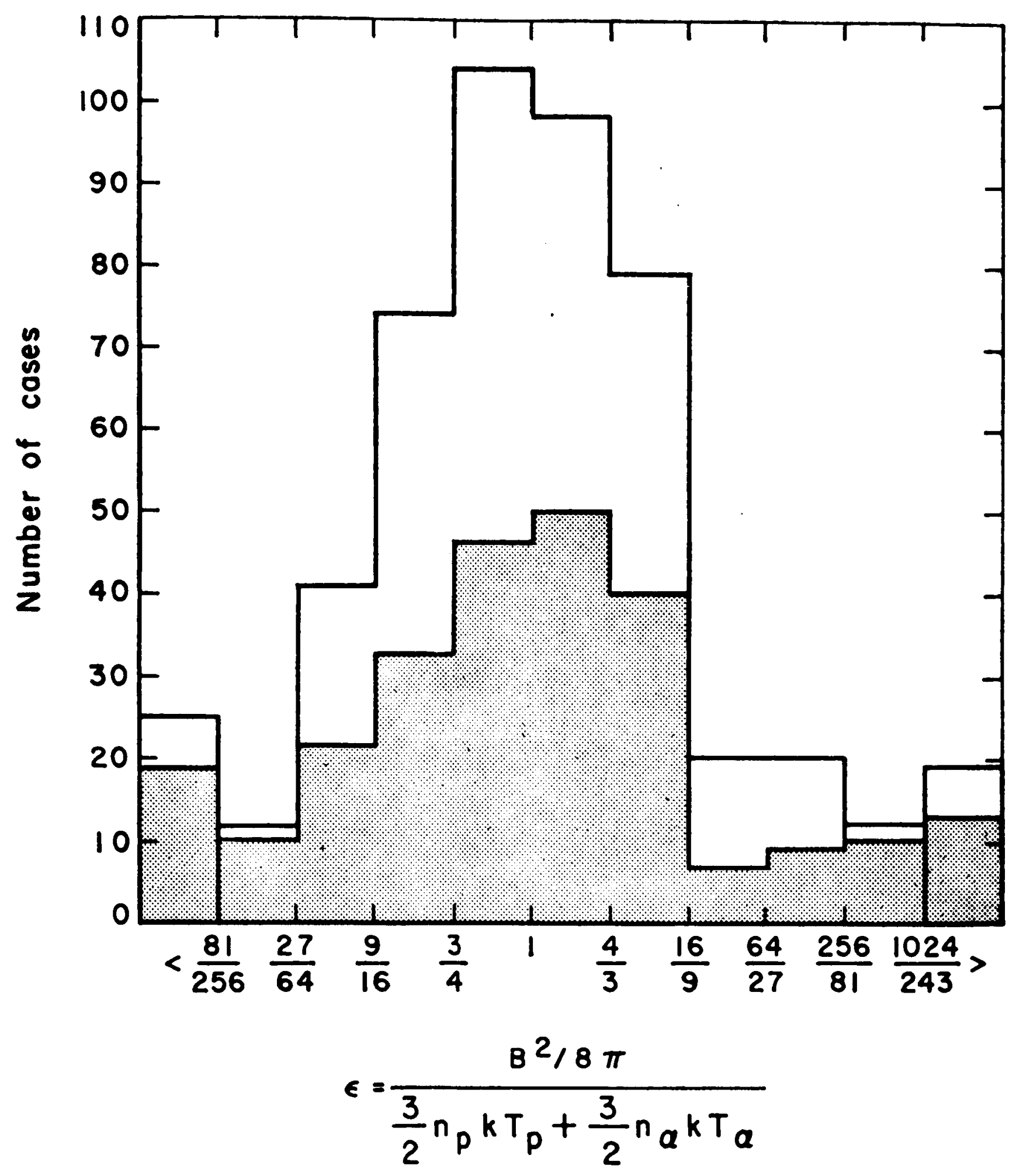

Figure 16 


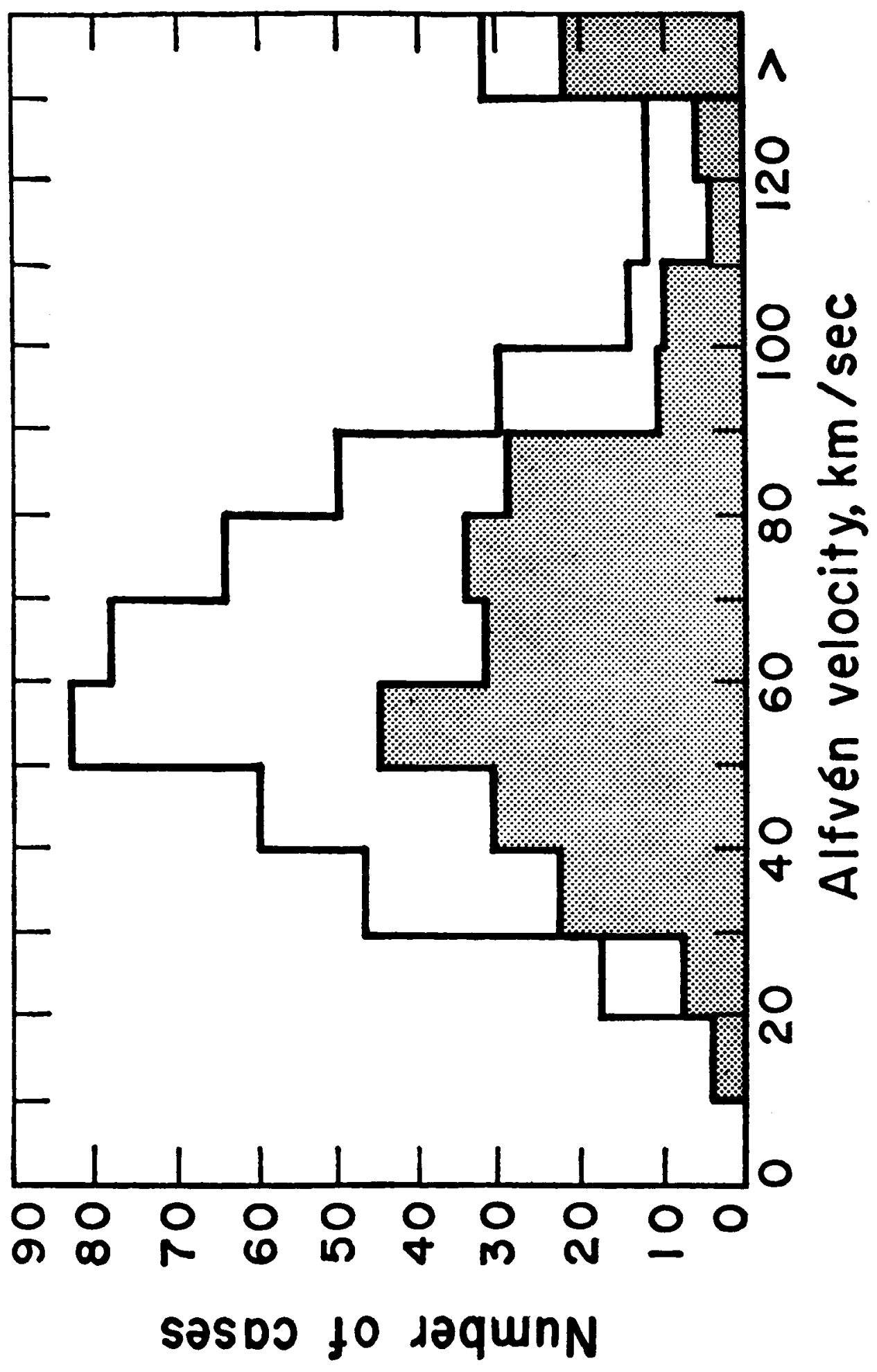

Figure 17 


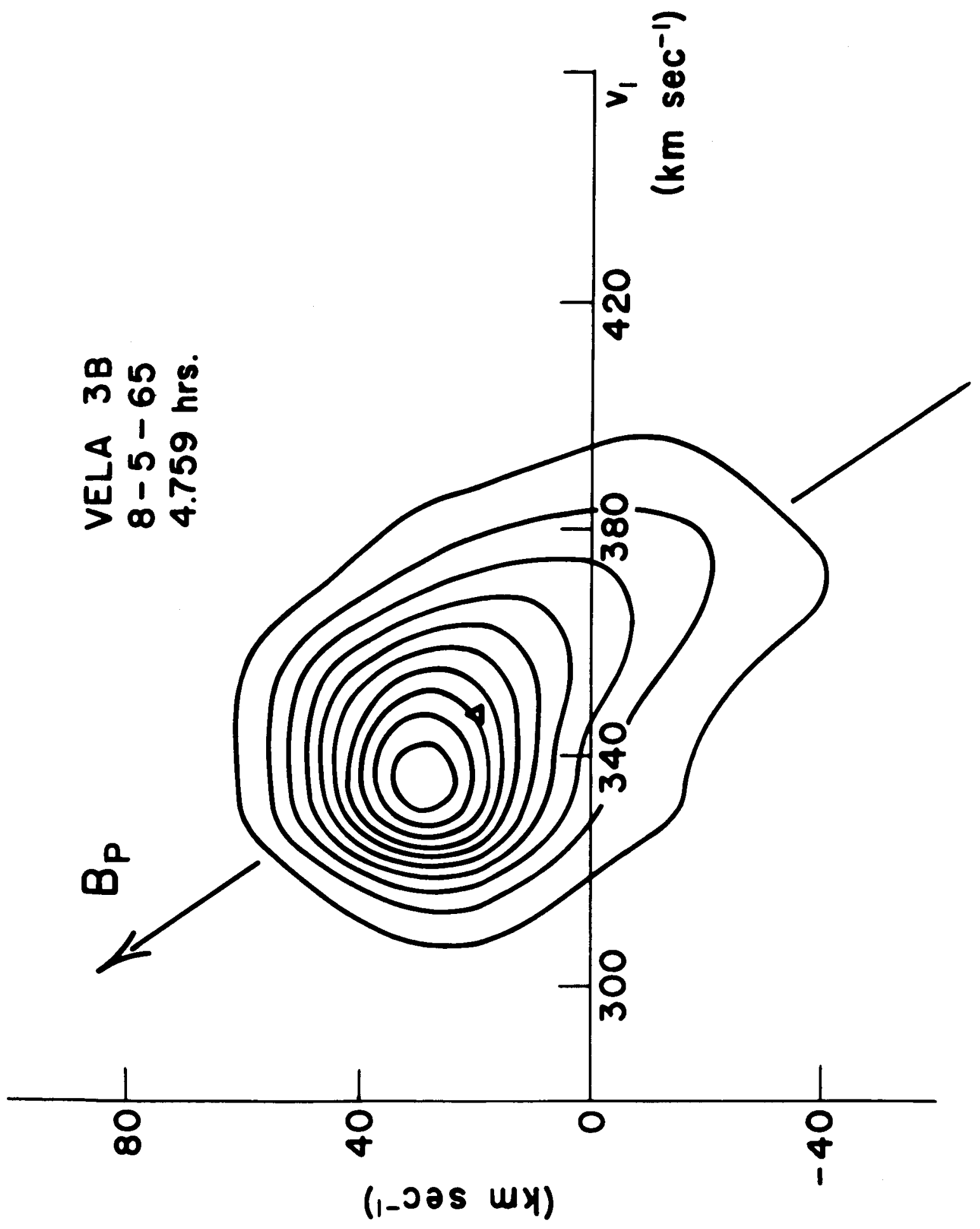

Figure 18 


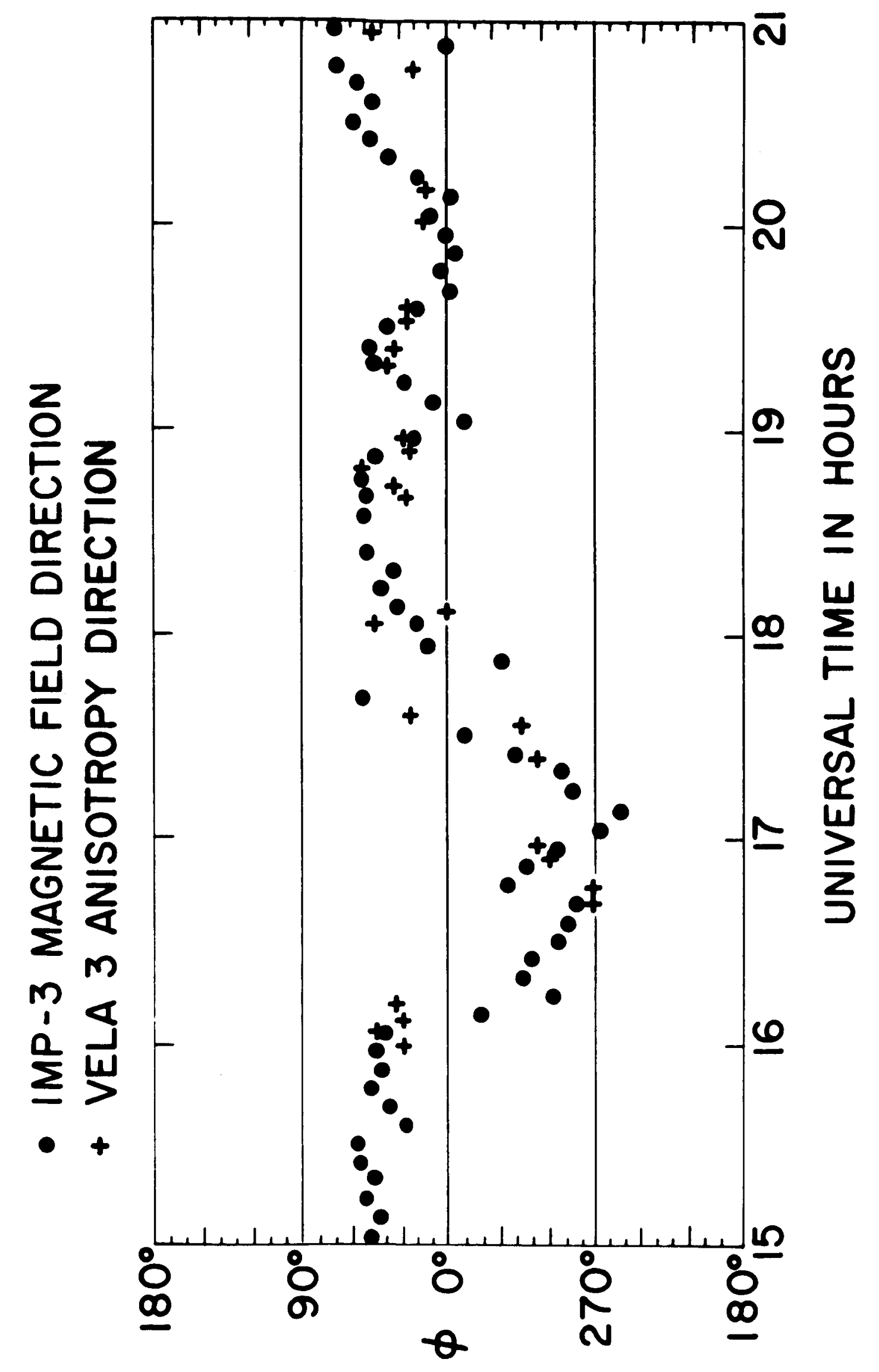

Figure 19 


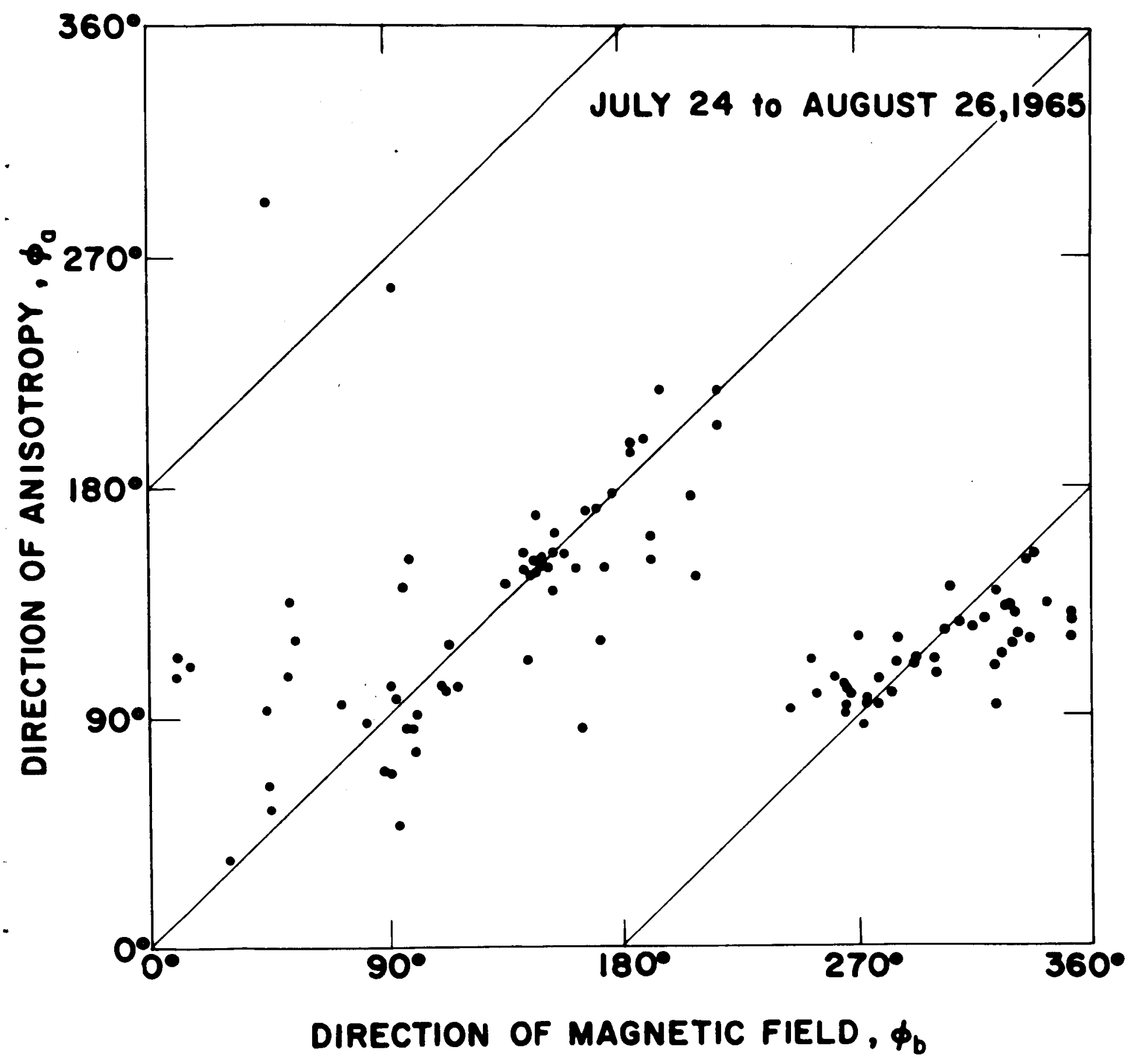

Figure 20 


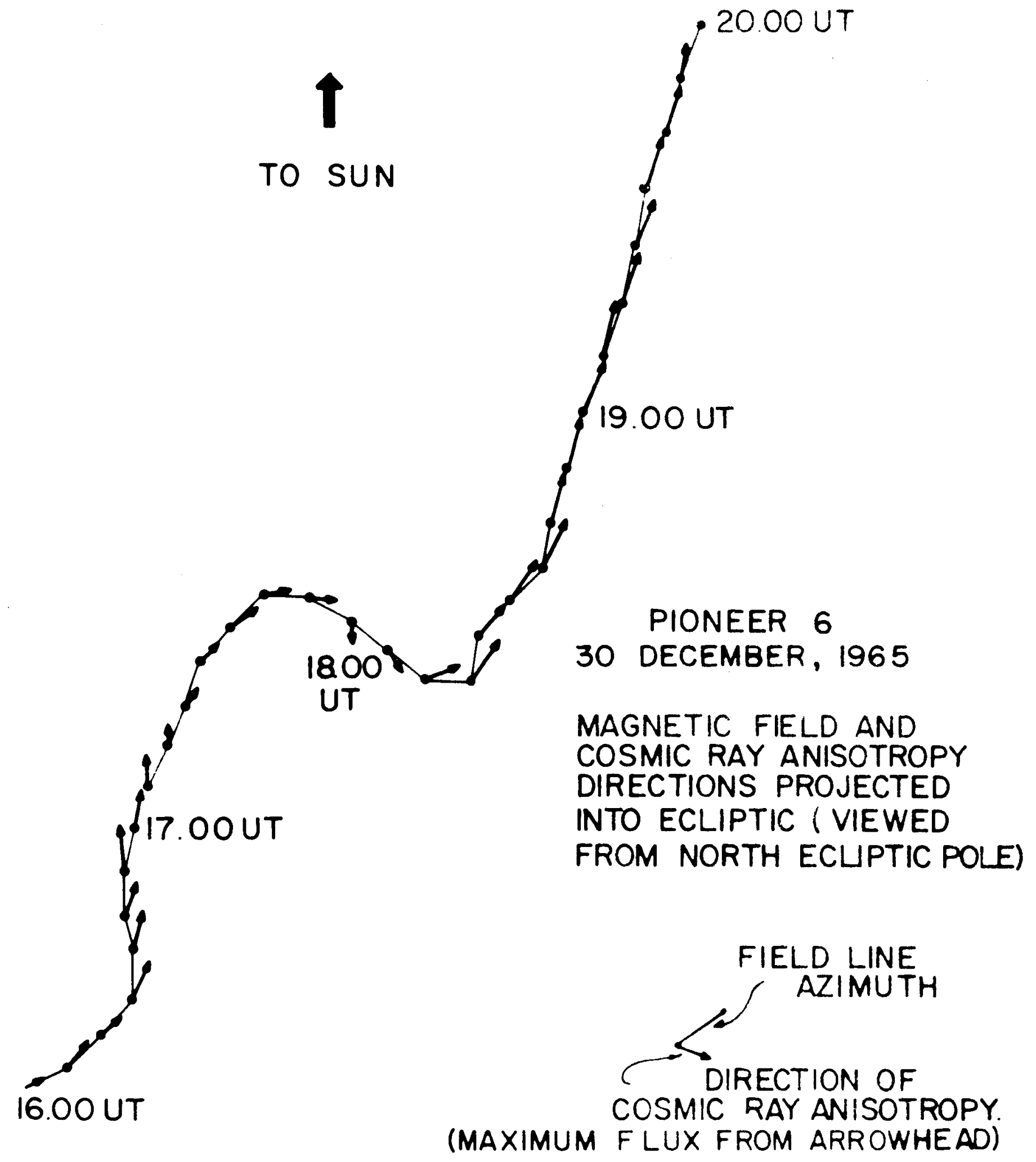




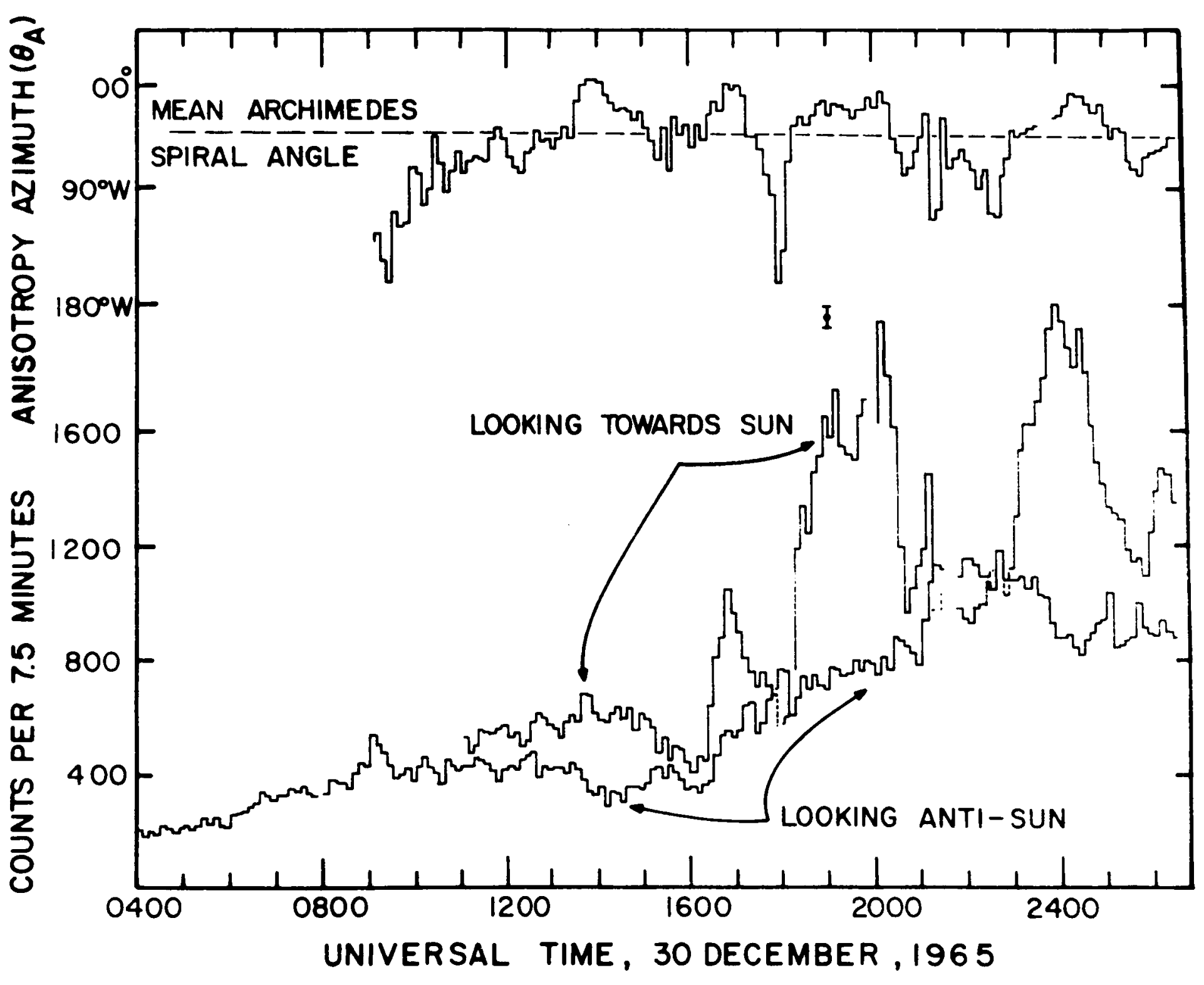




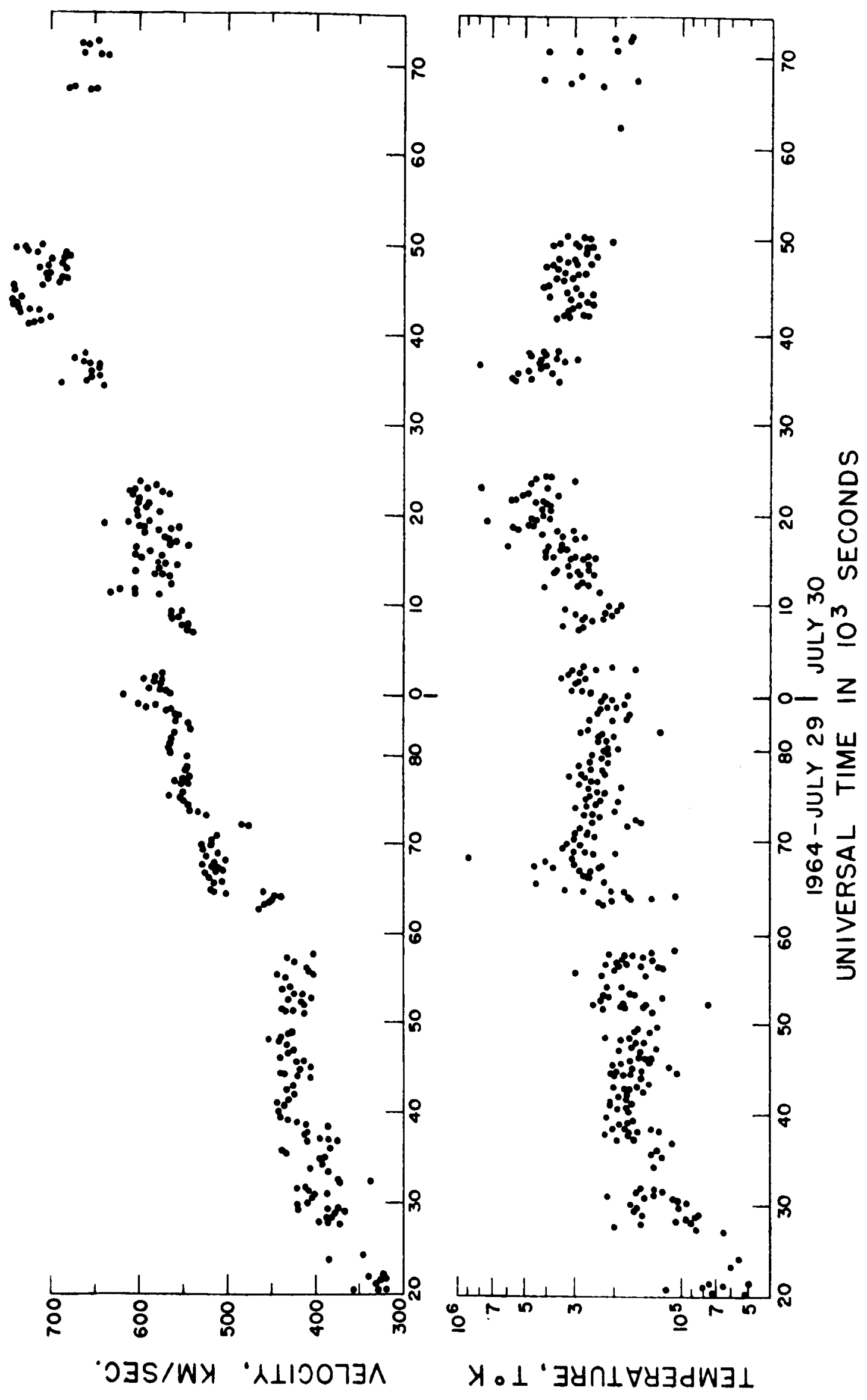

Figure 23 


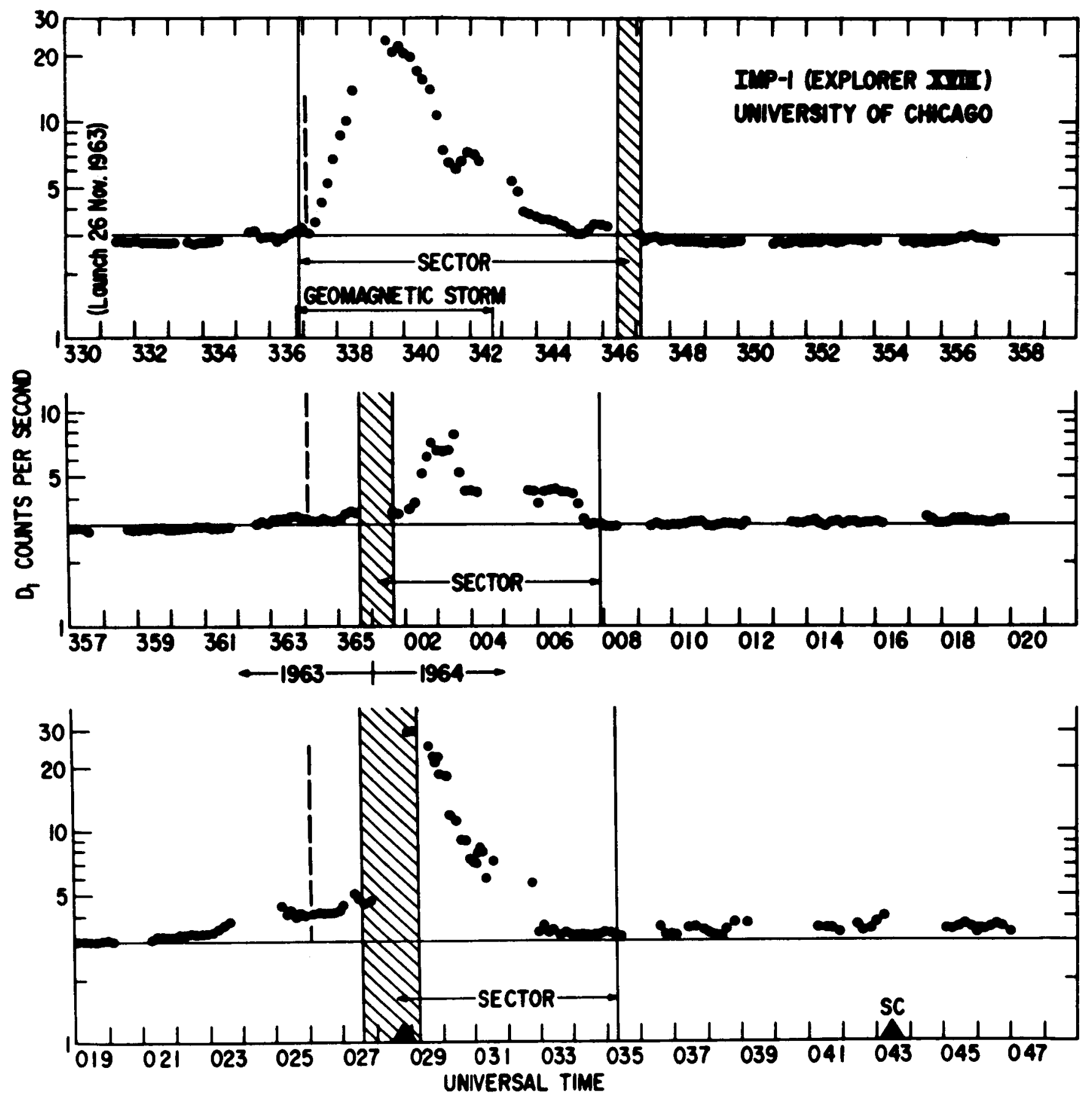

Figure 24 

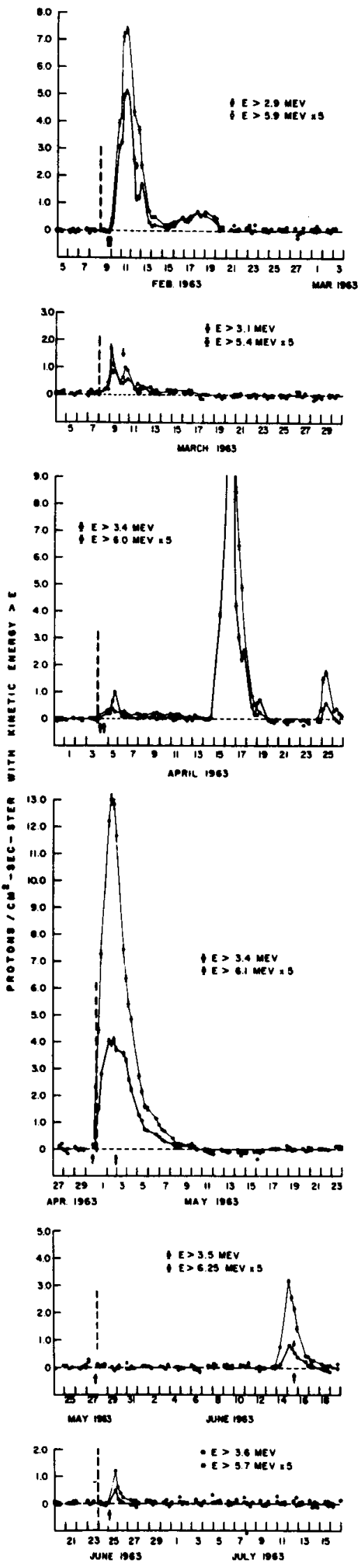


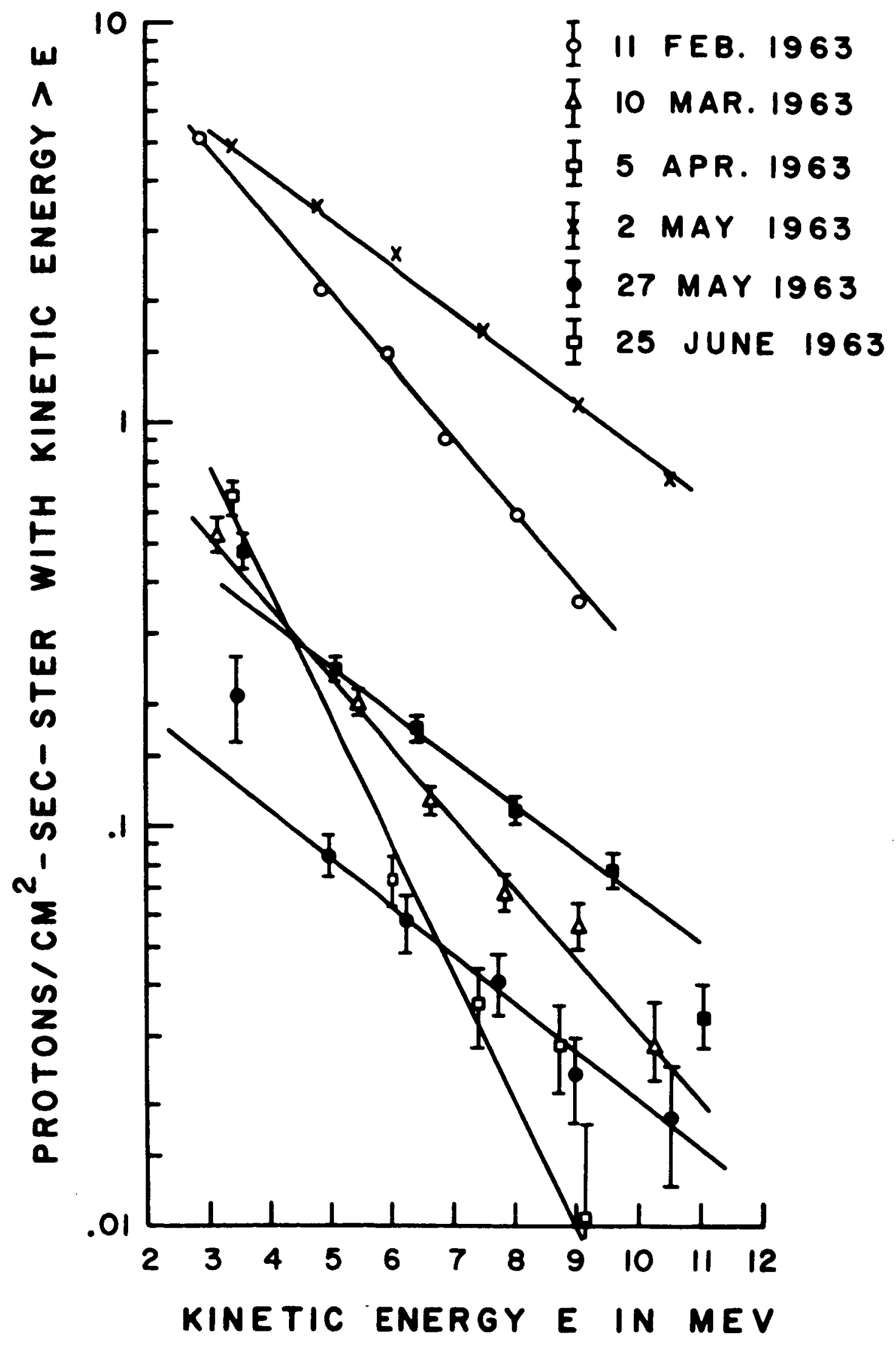




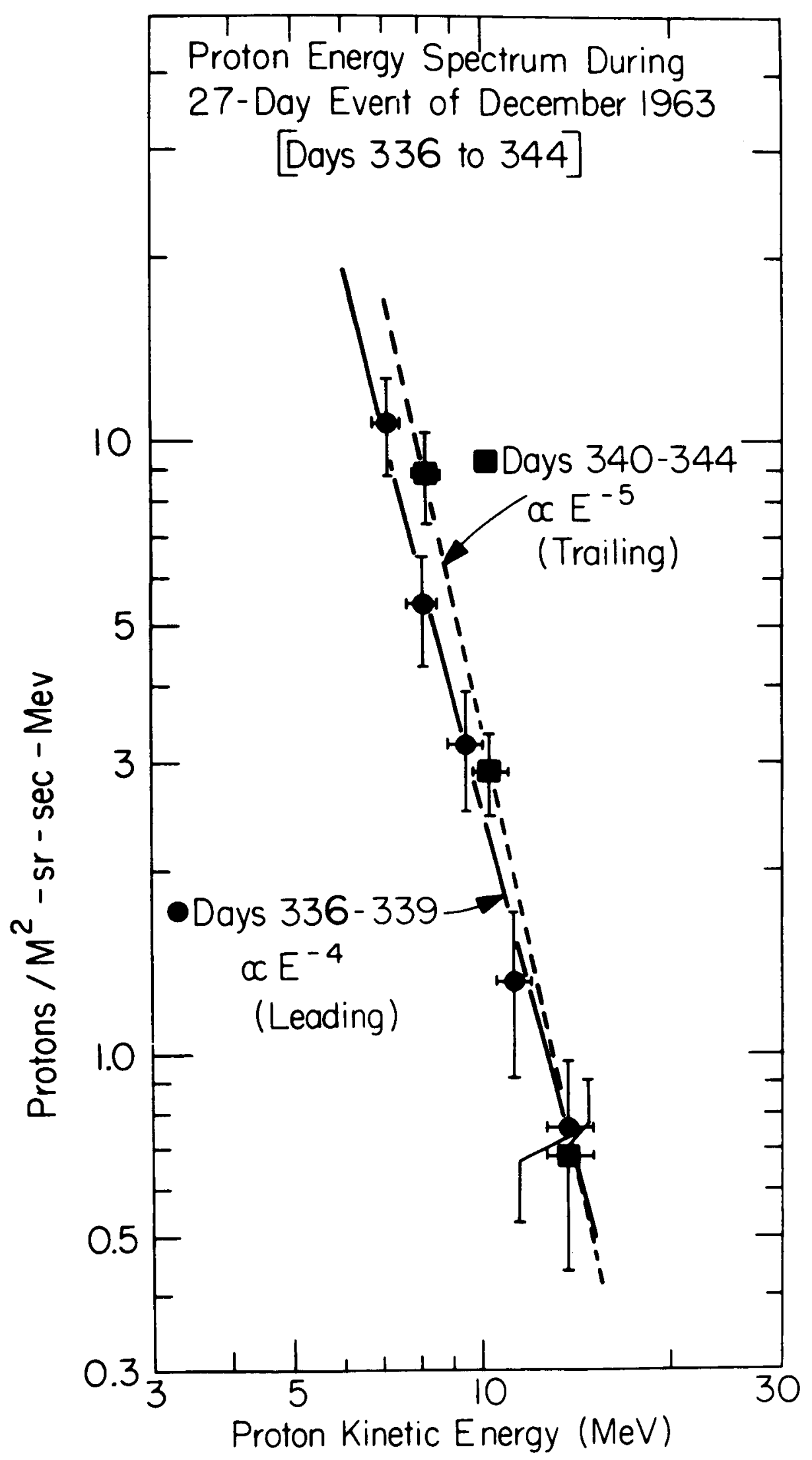




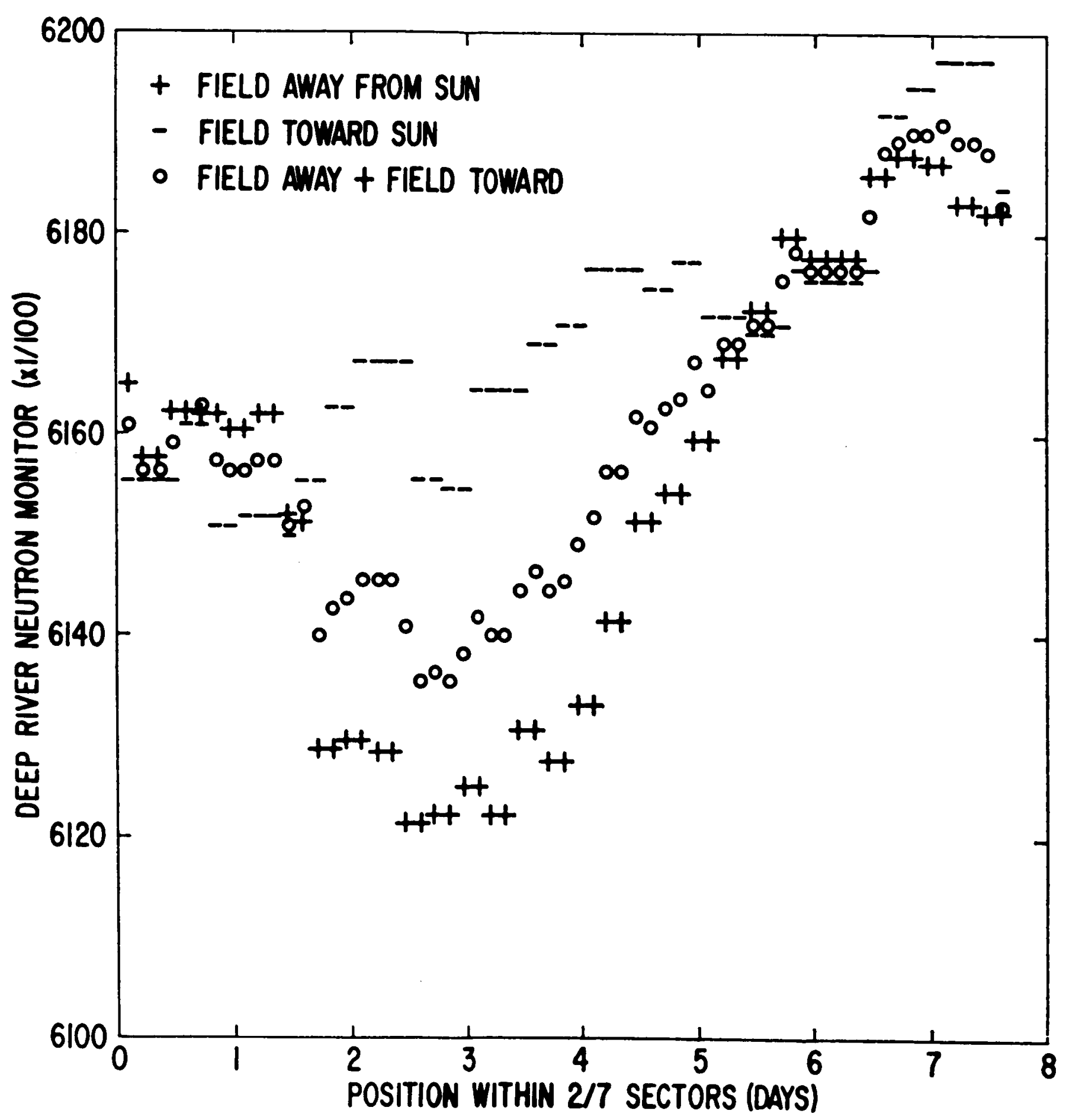

Figure 28 


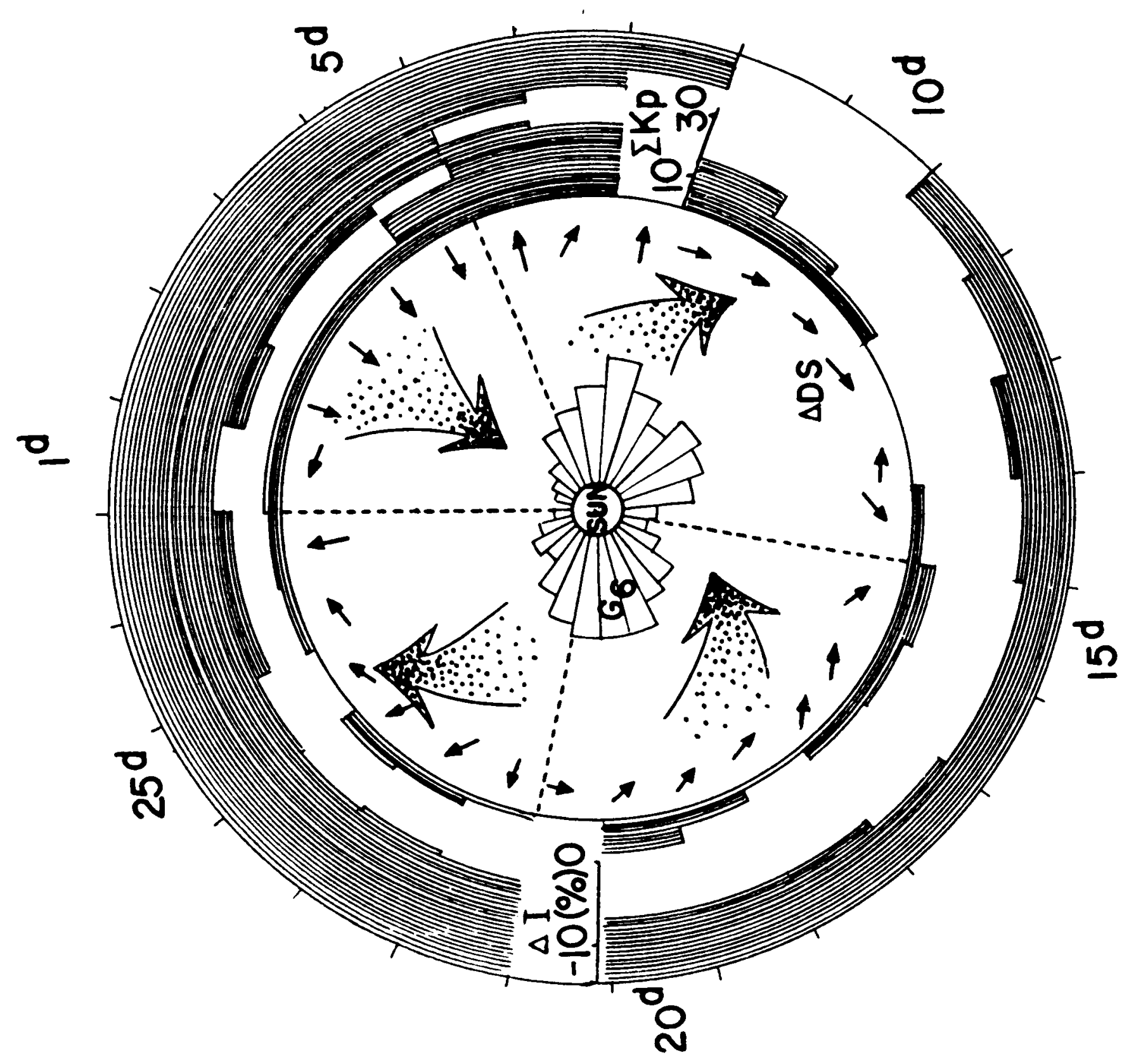




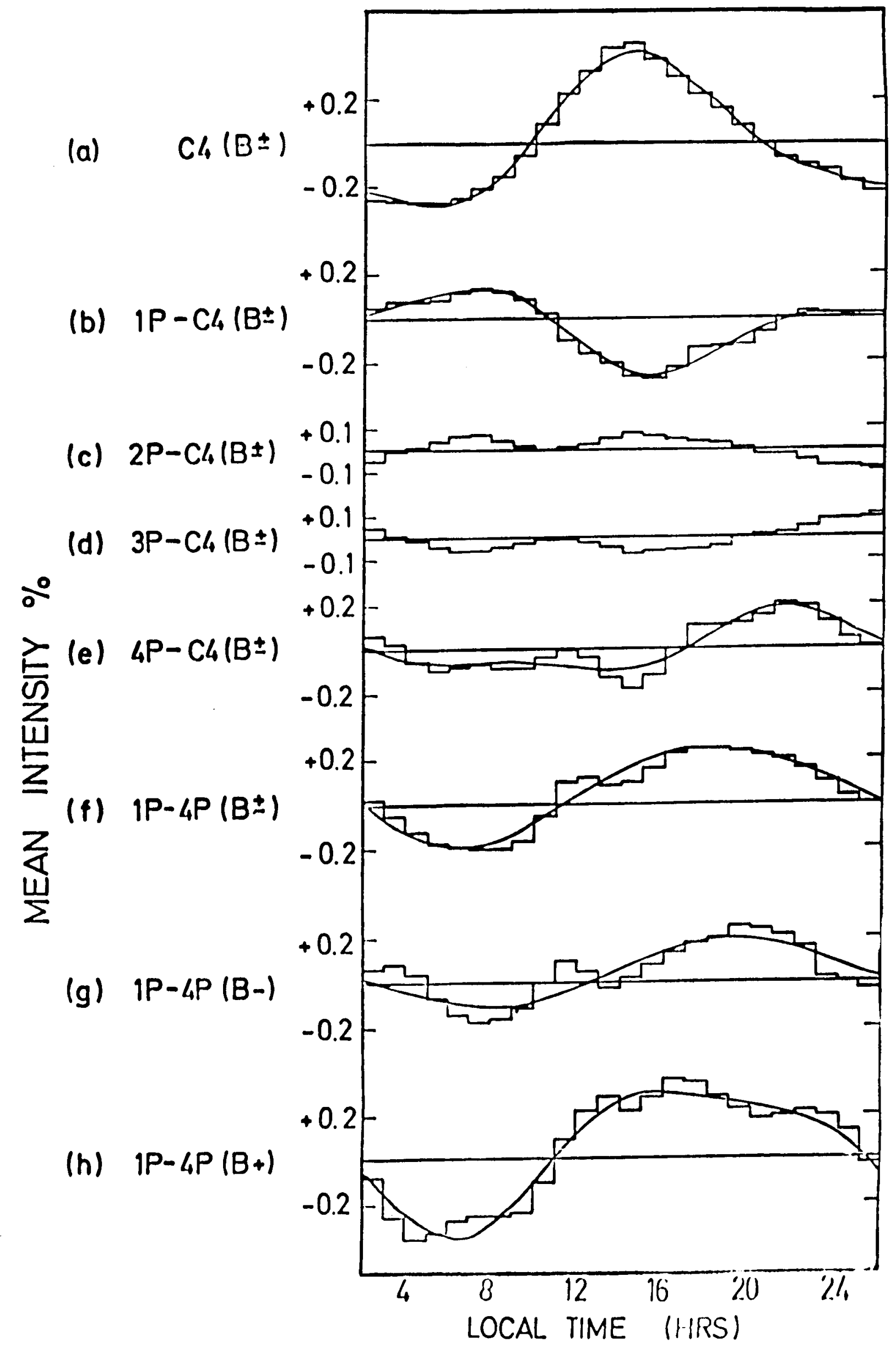

Figure 30 


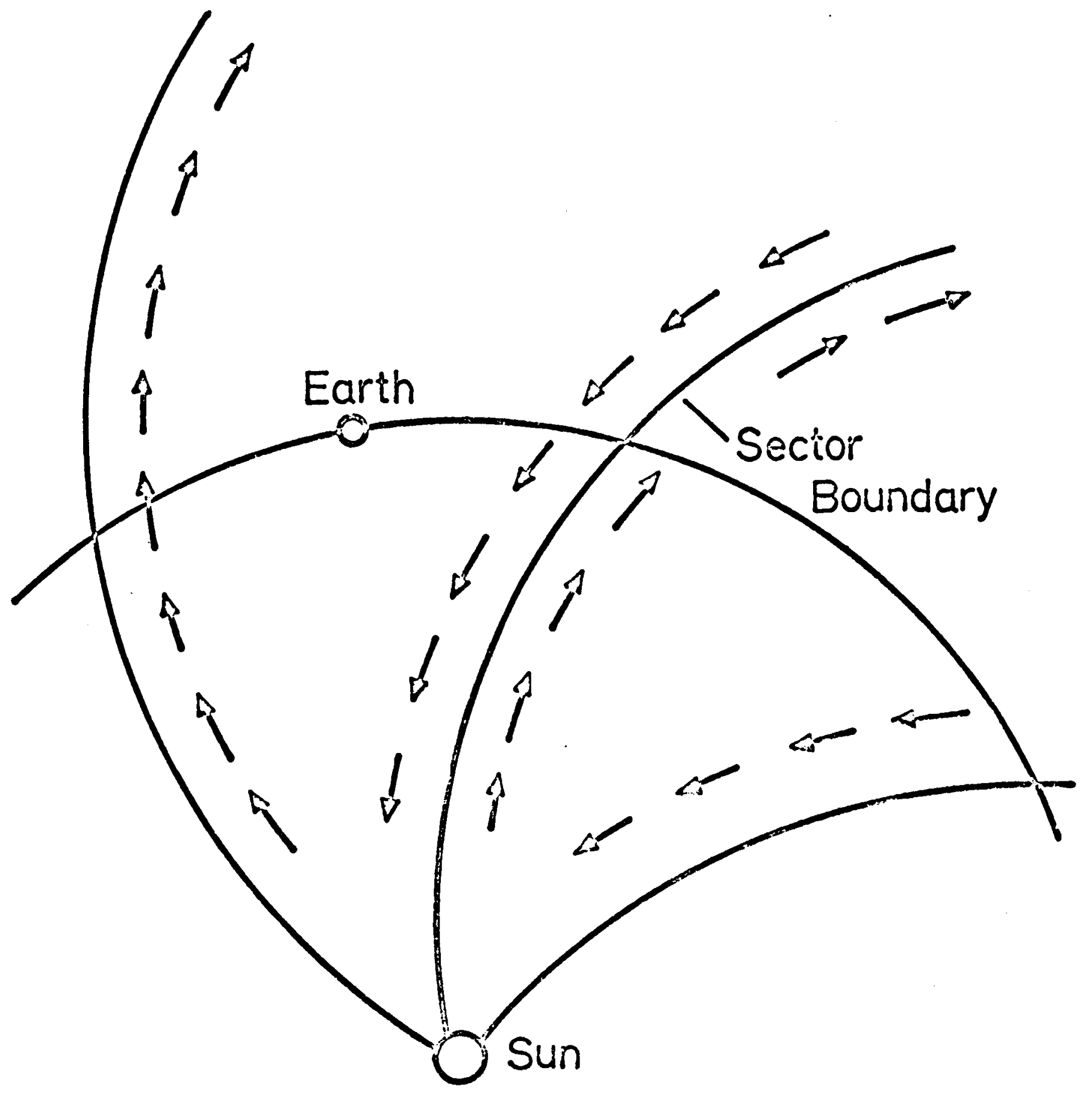

Figure 31 


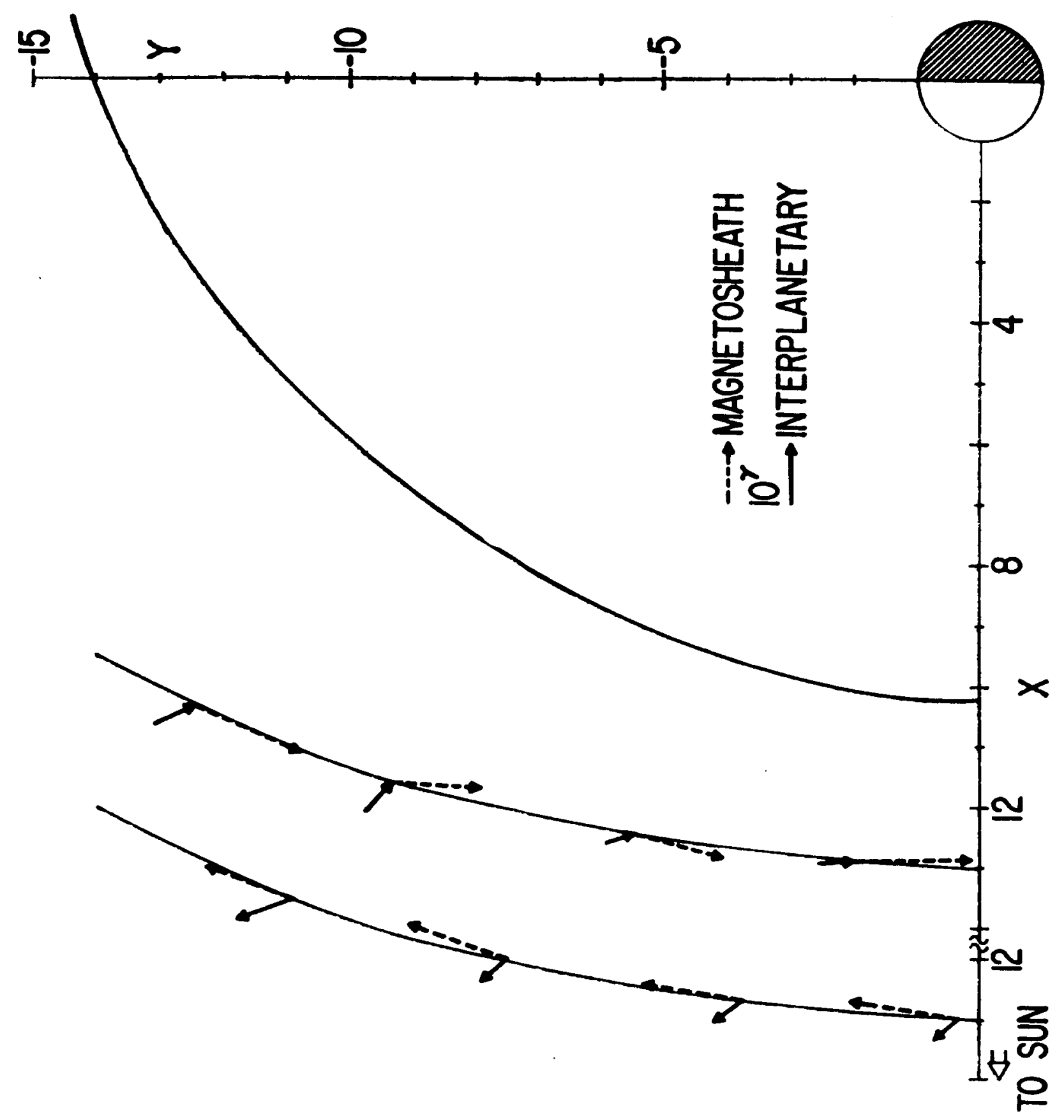

Figure 32 
ONOJ $J S / S \perp N \cap O J \perp \exists N-I I N N R N I$

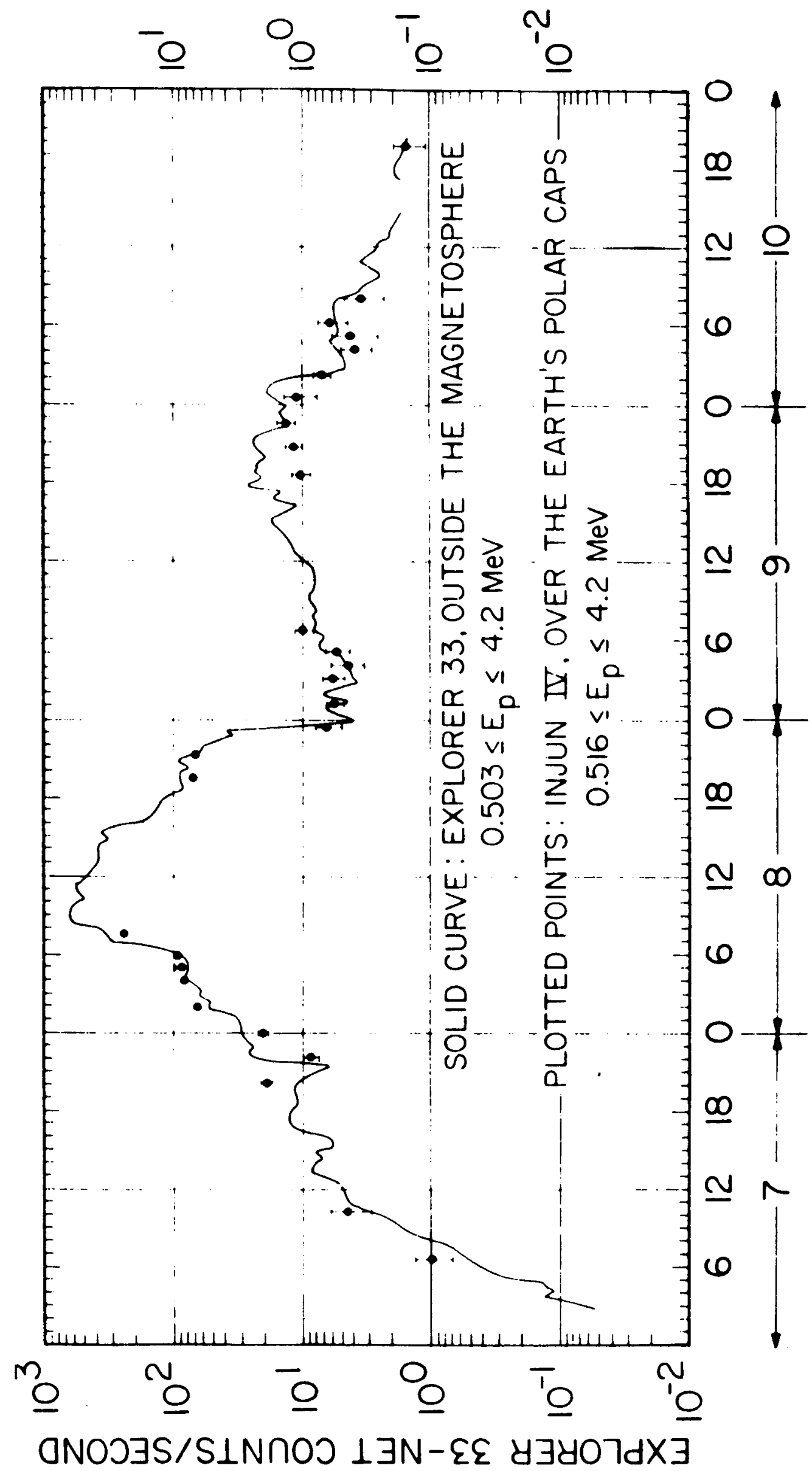

Figure 33 


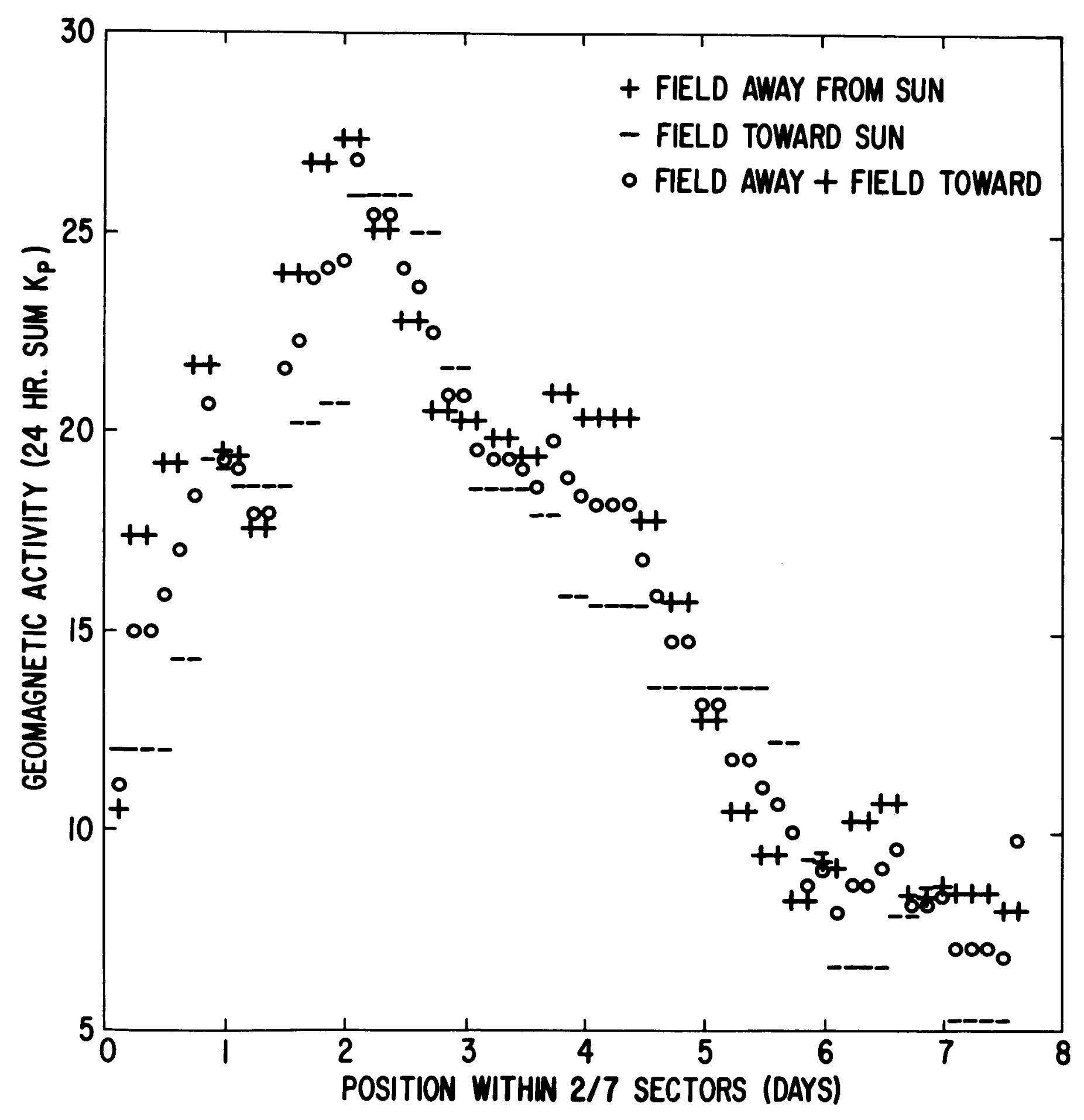




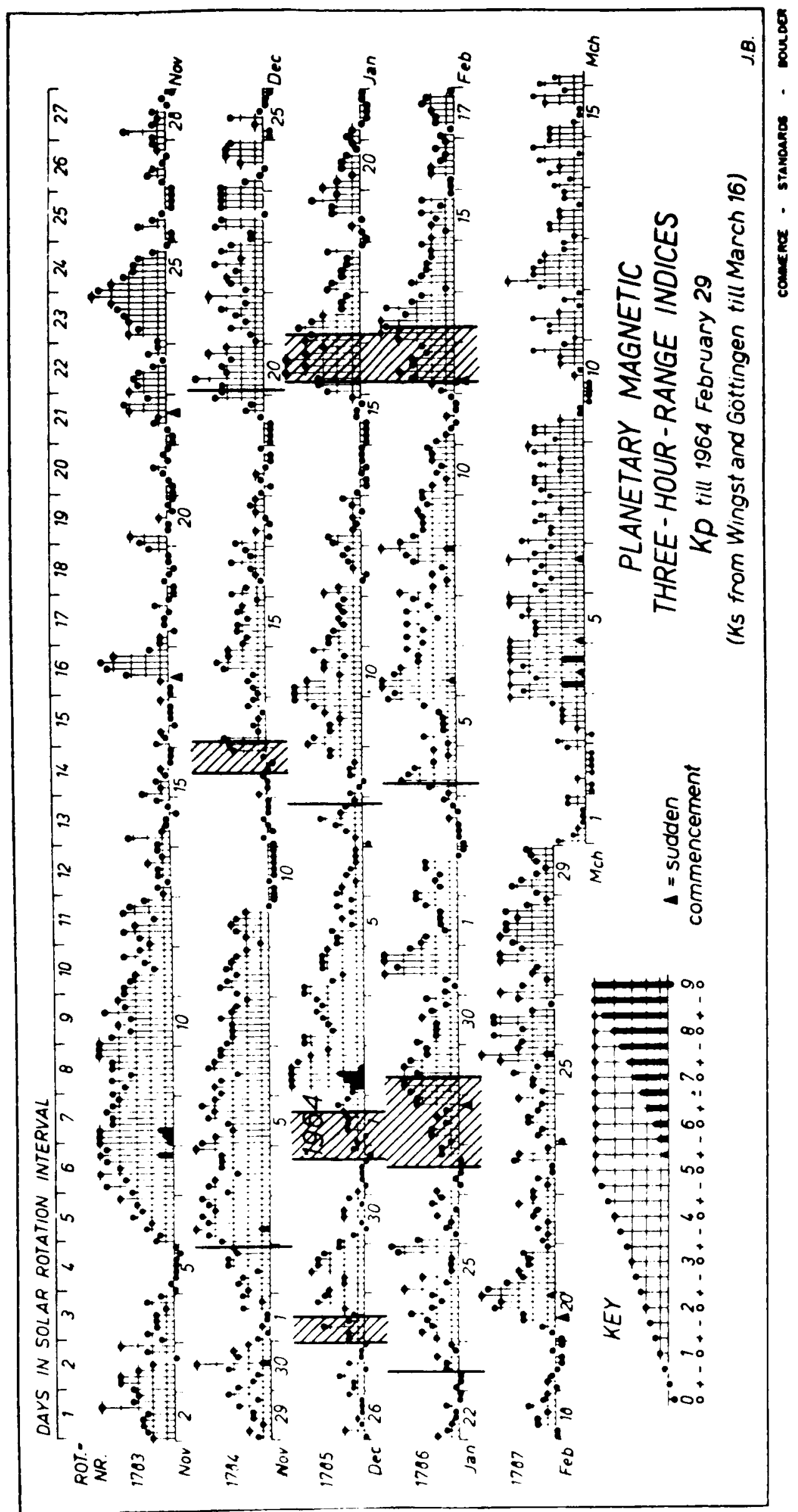




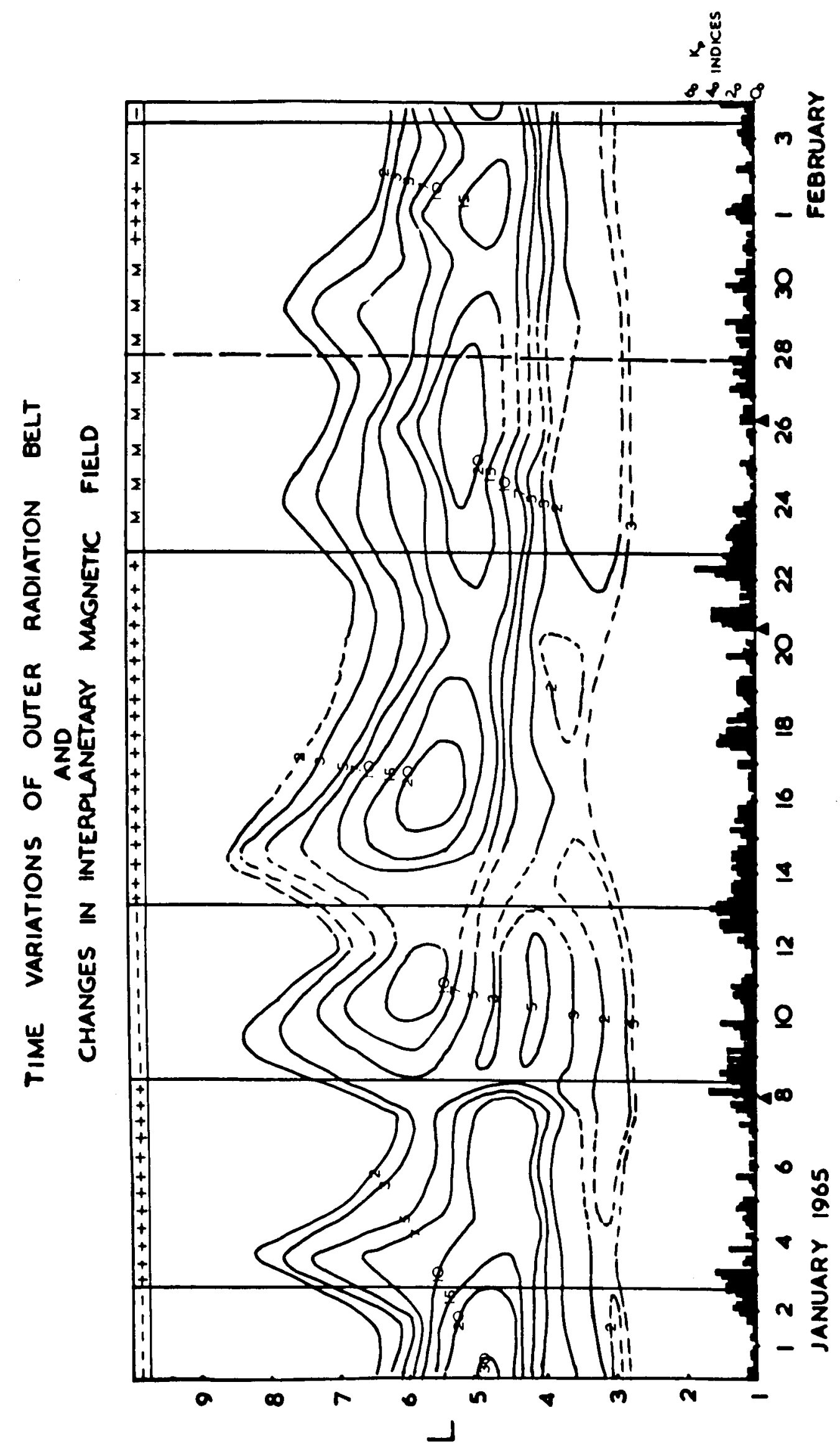

Figure 36 

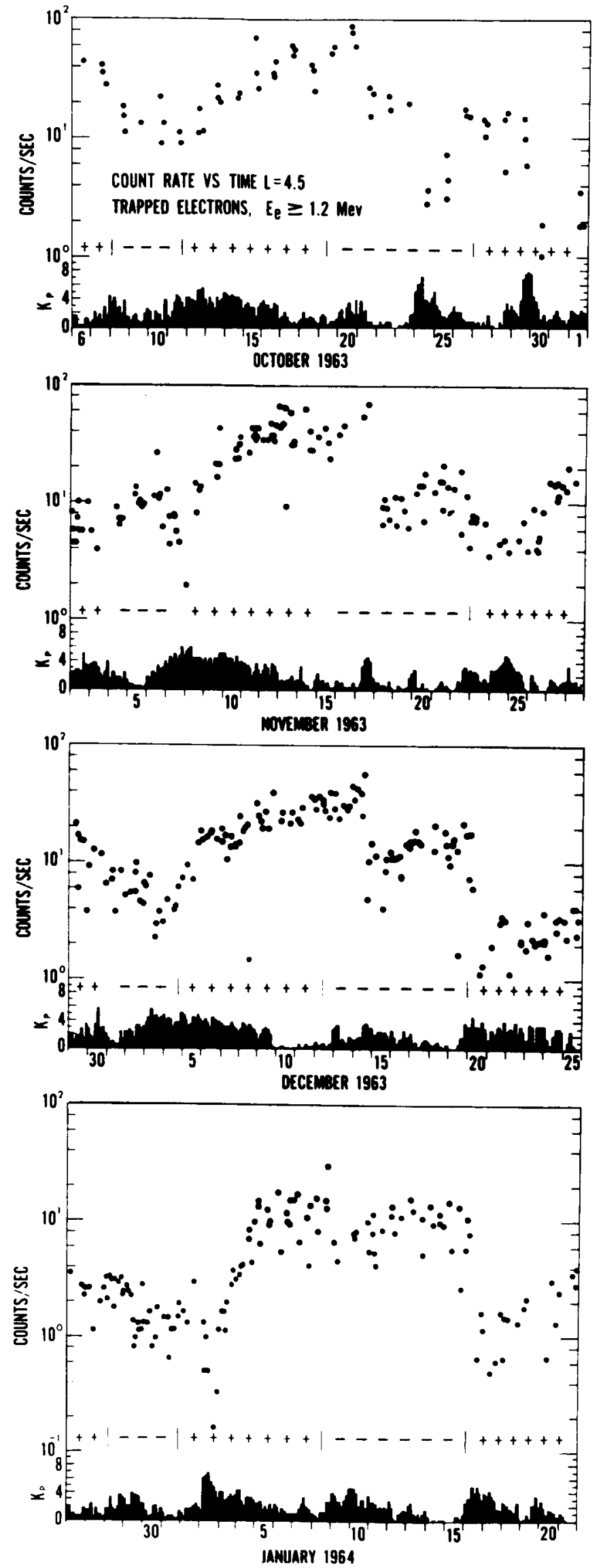

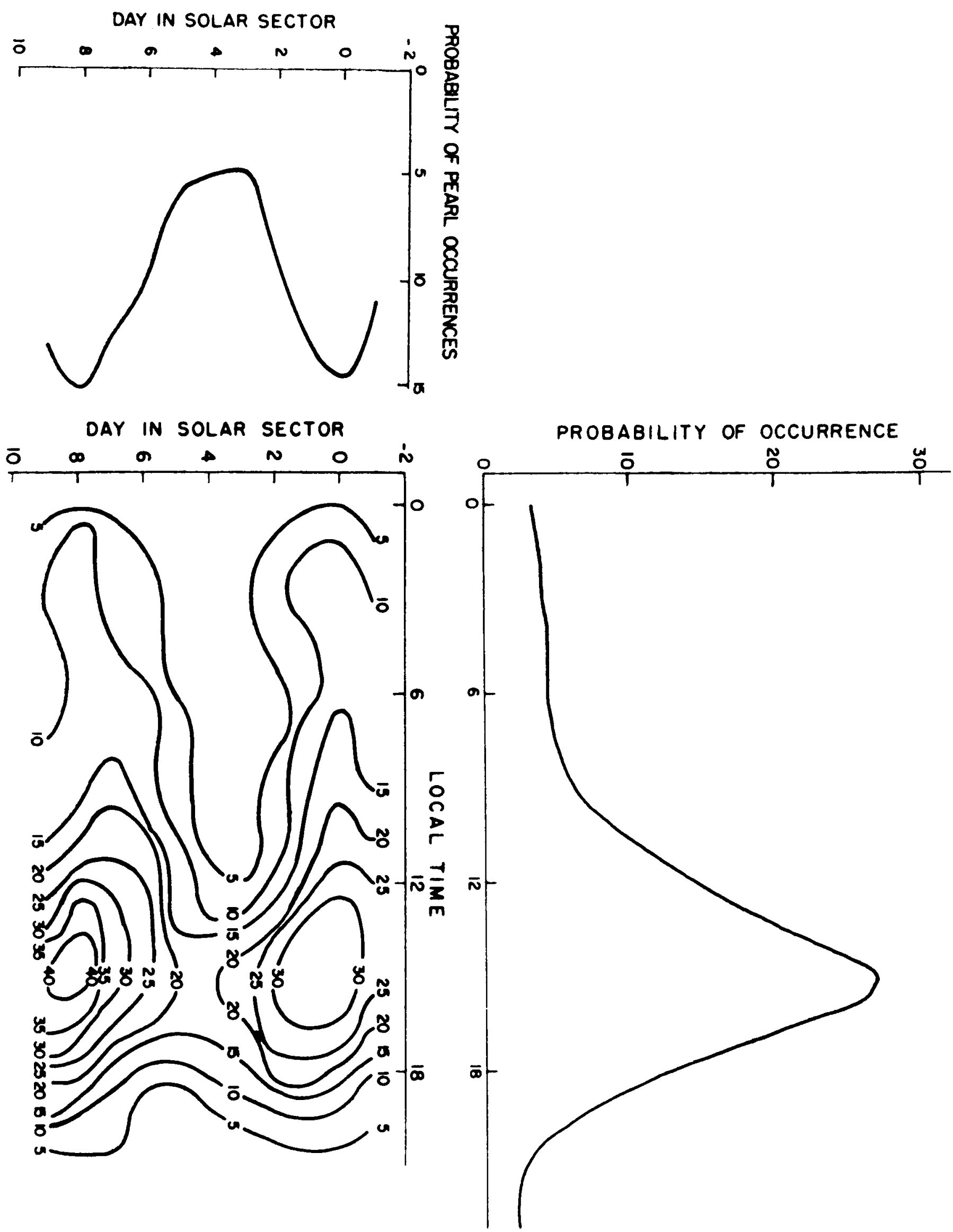

Figure 38 


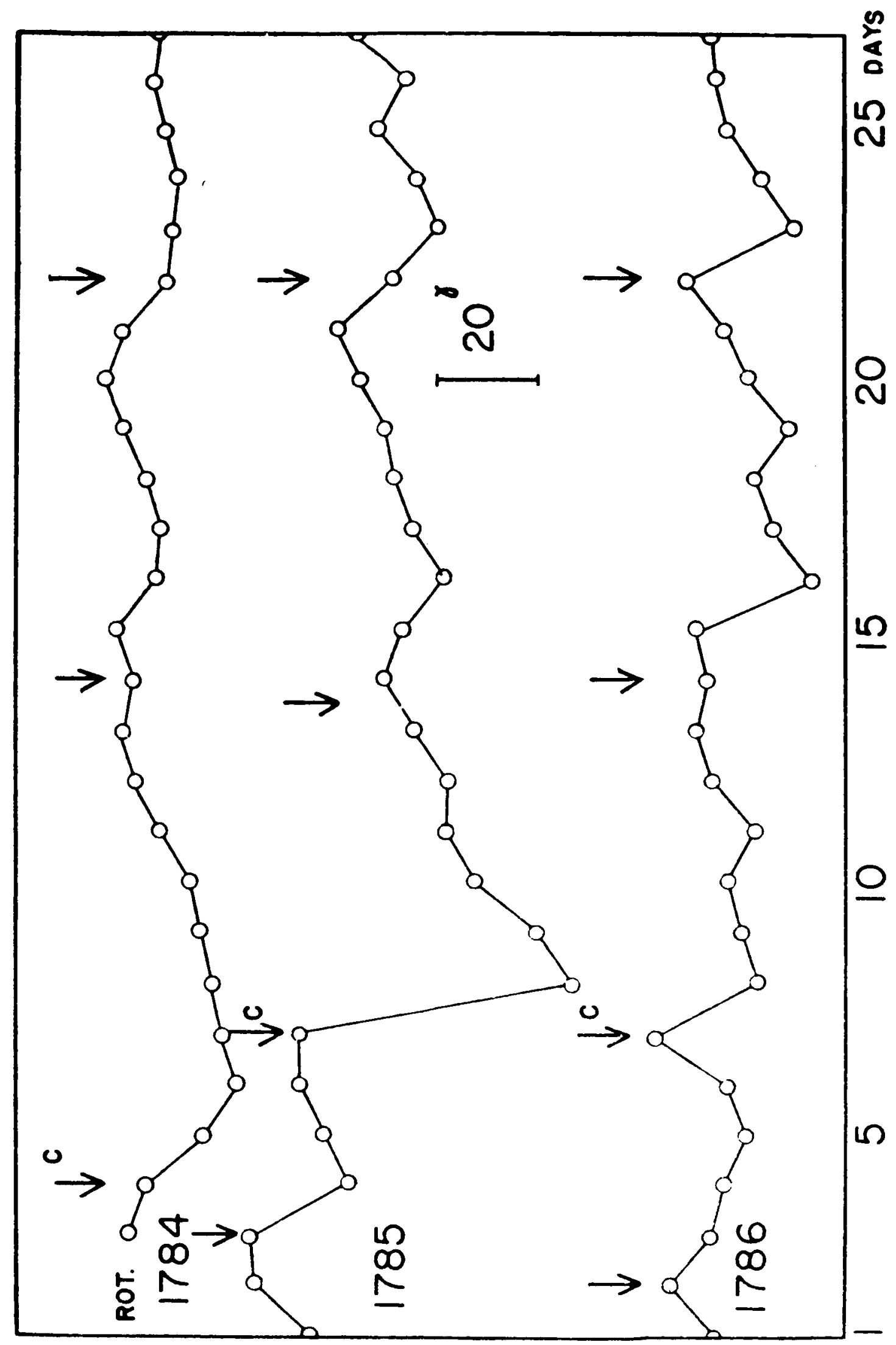

Figure 39 


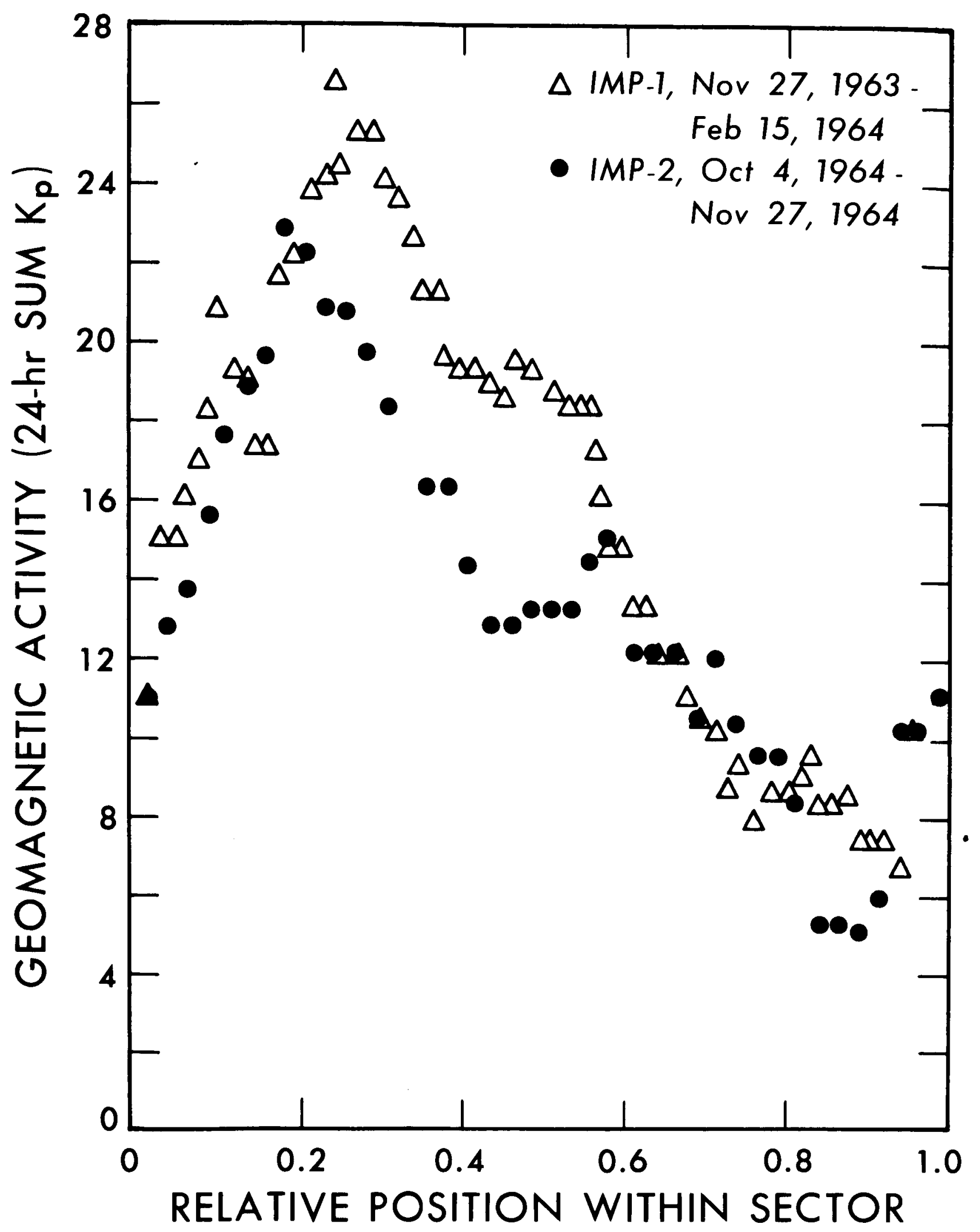




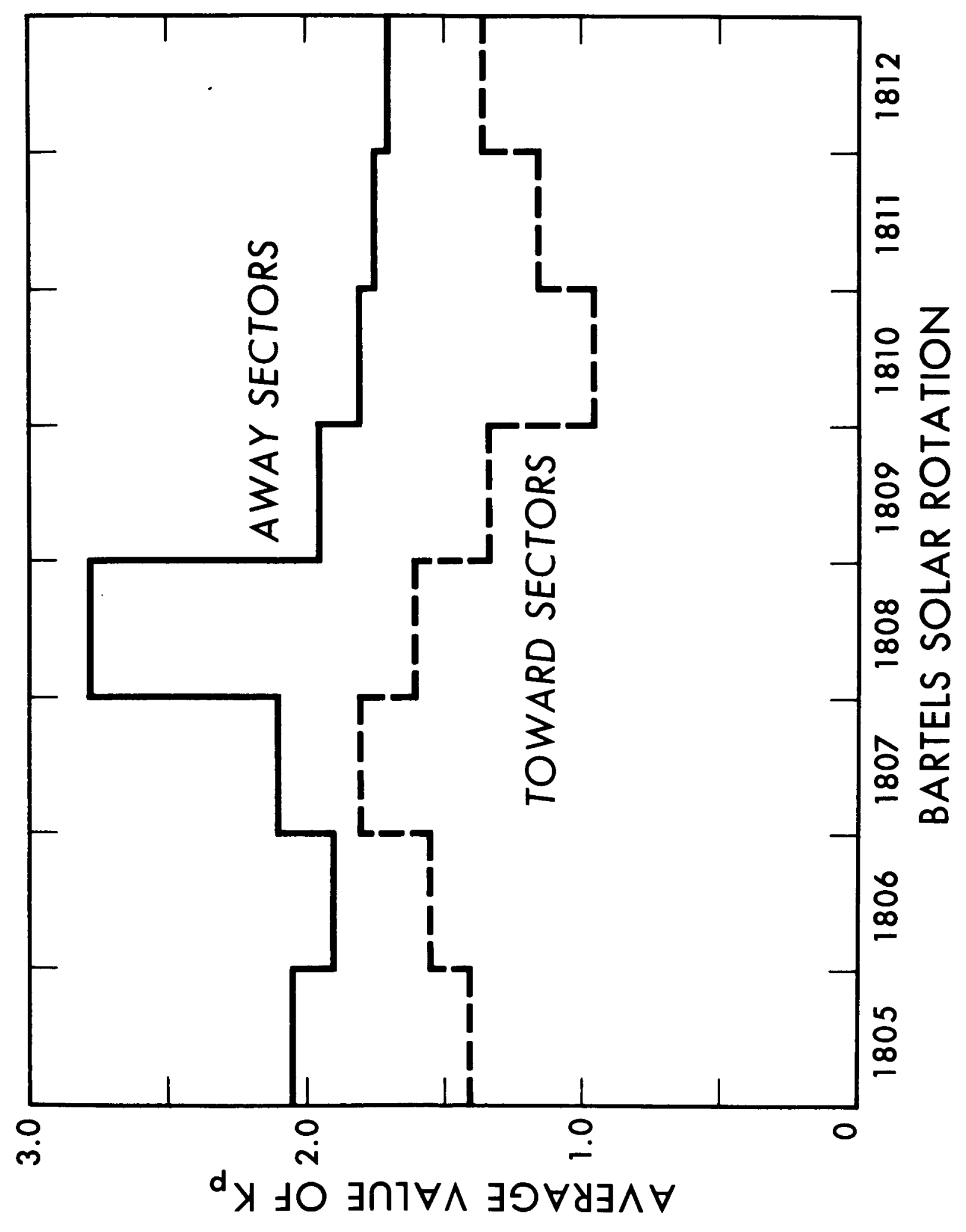

Figure 41 

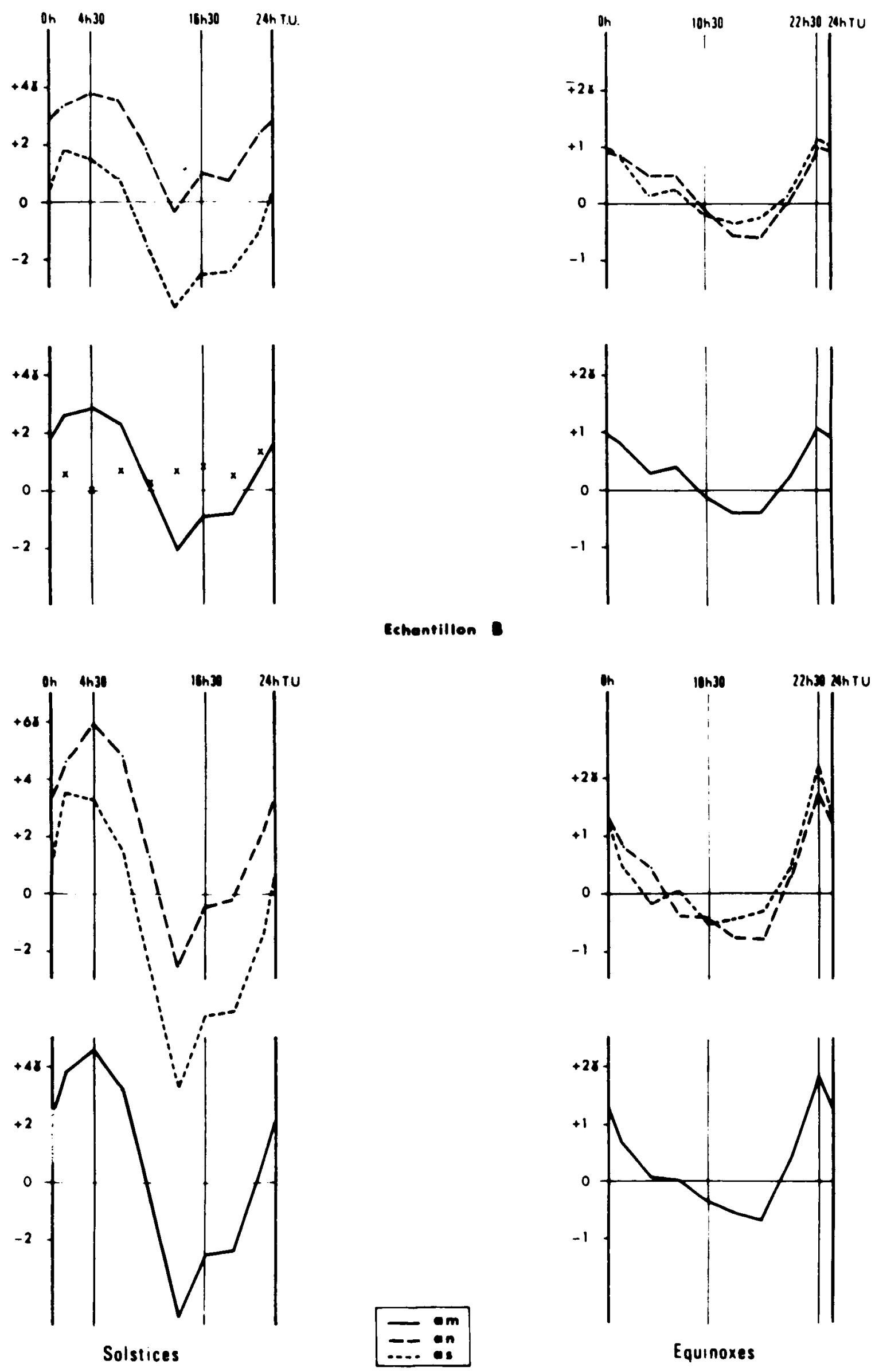

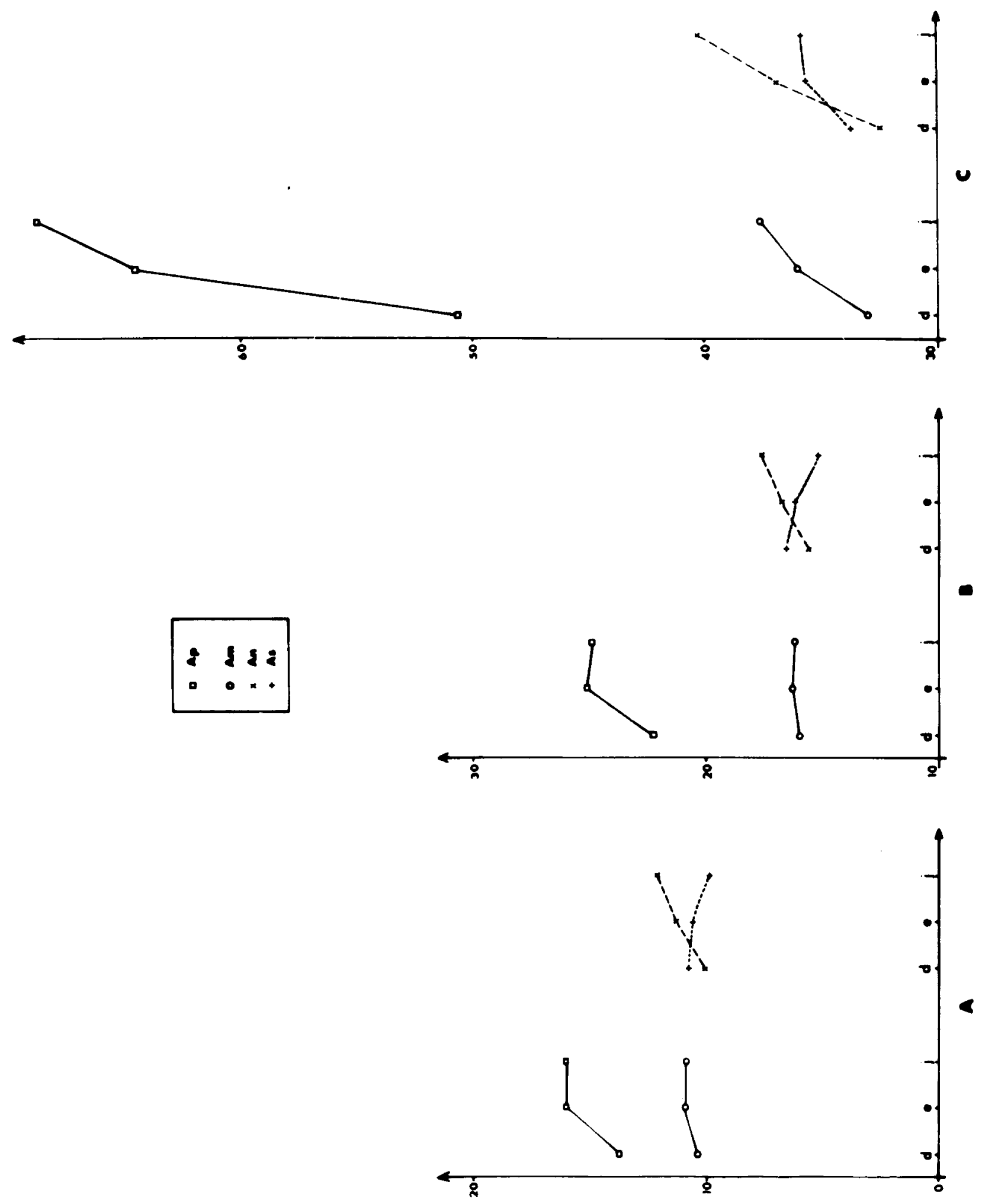

Figure 43 


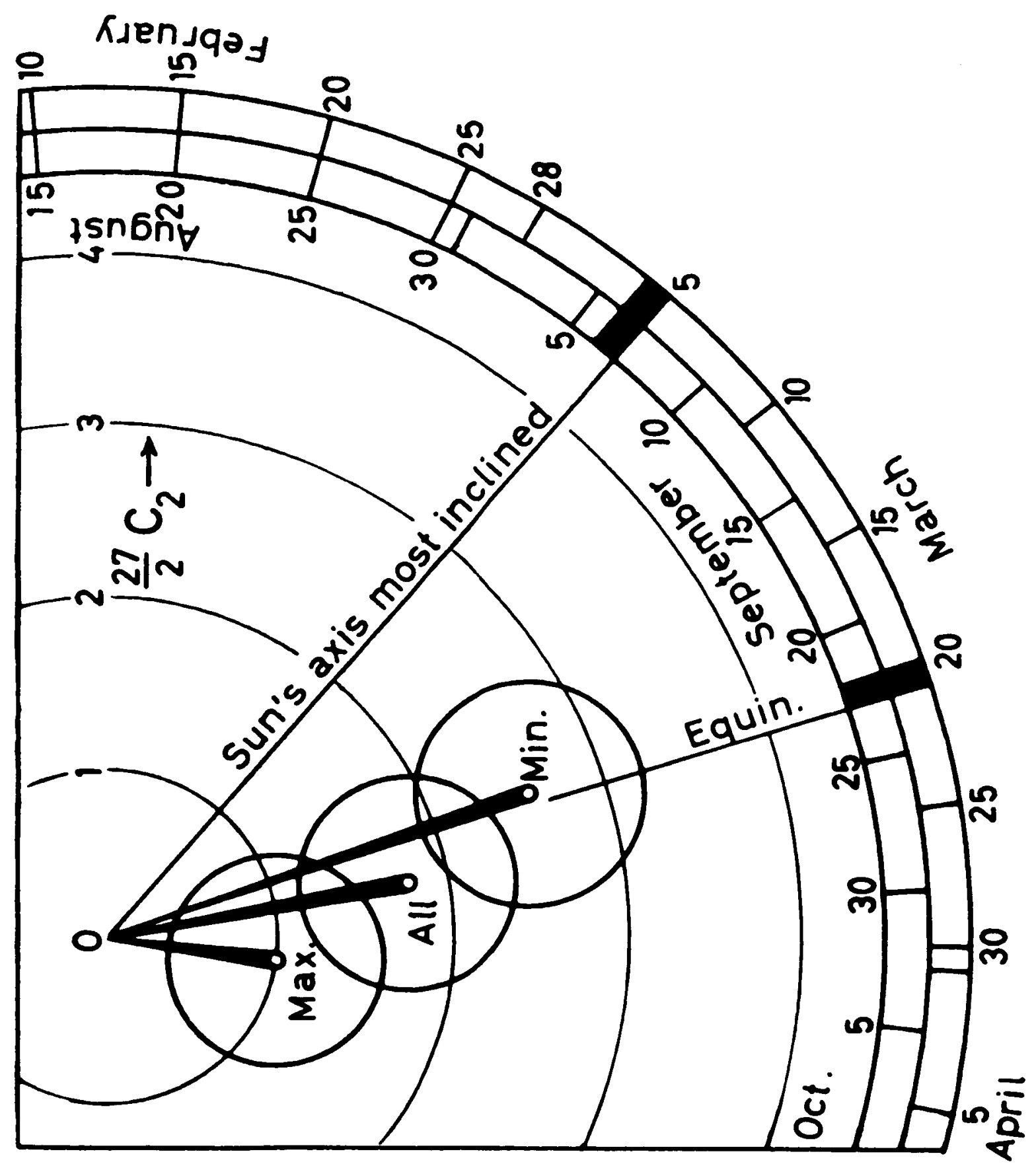

Figure 44 


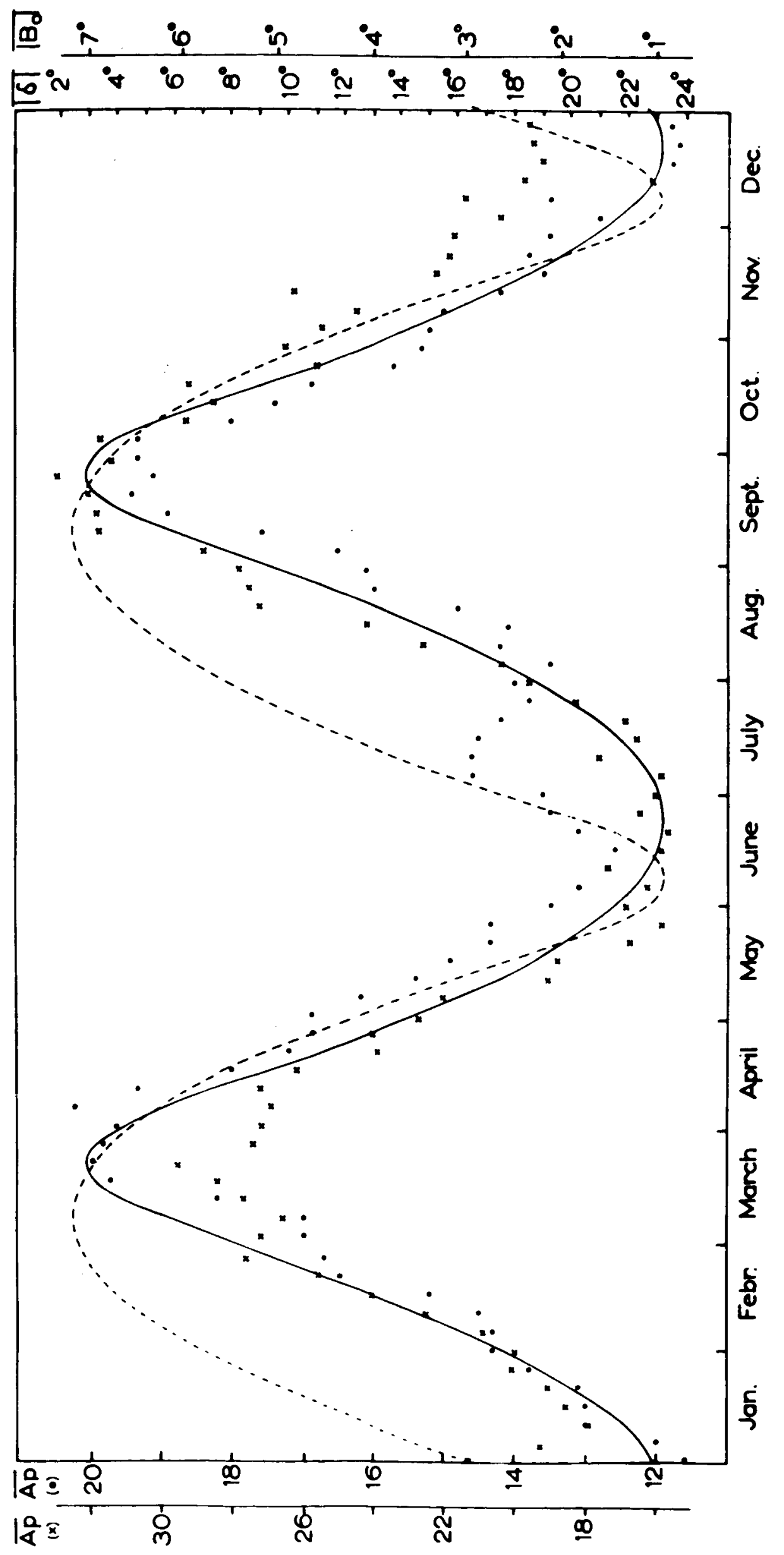

Figure 45 


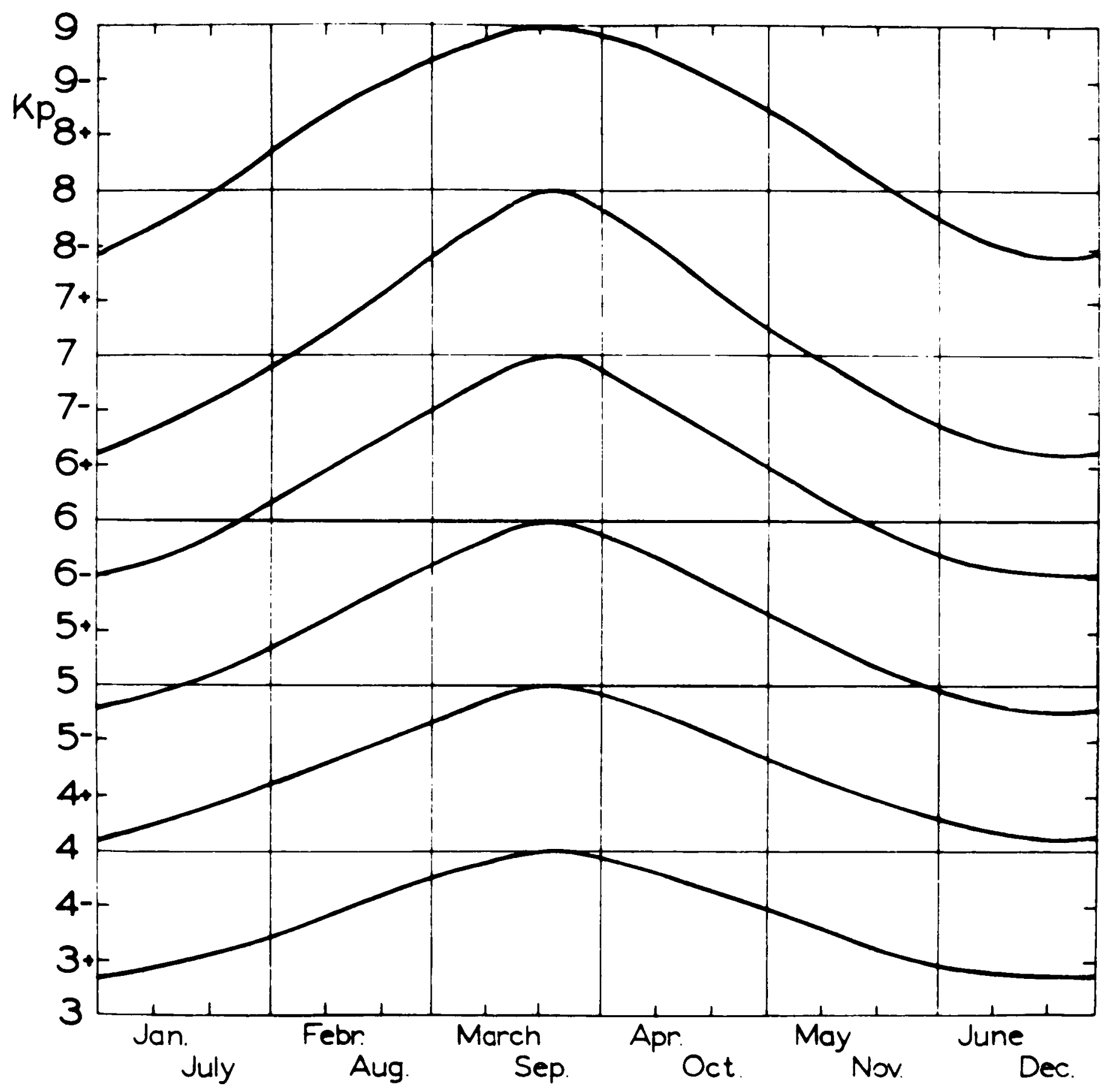

Figure 46 


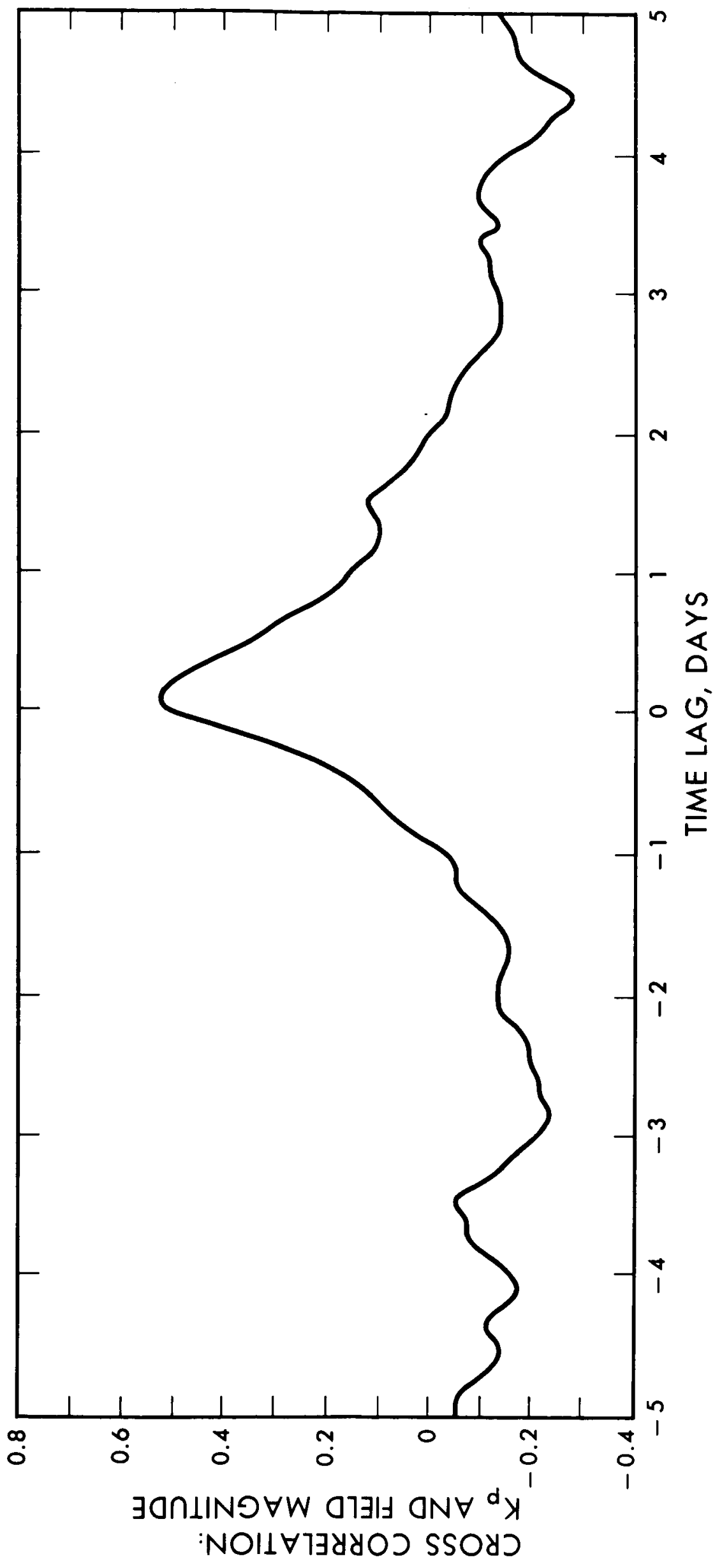

Figure 47 


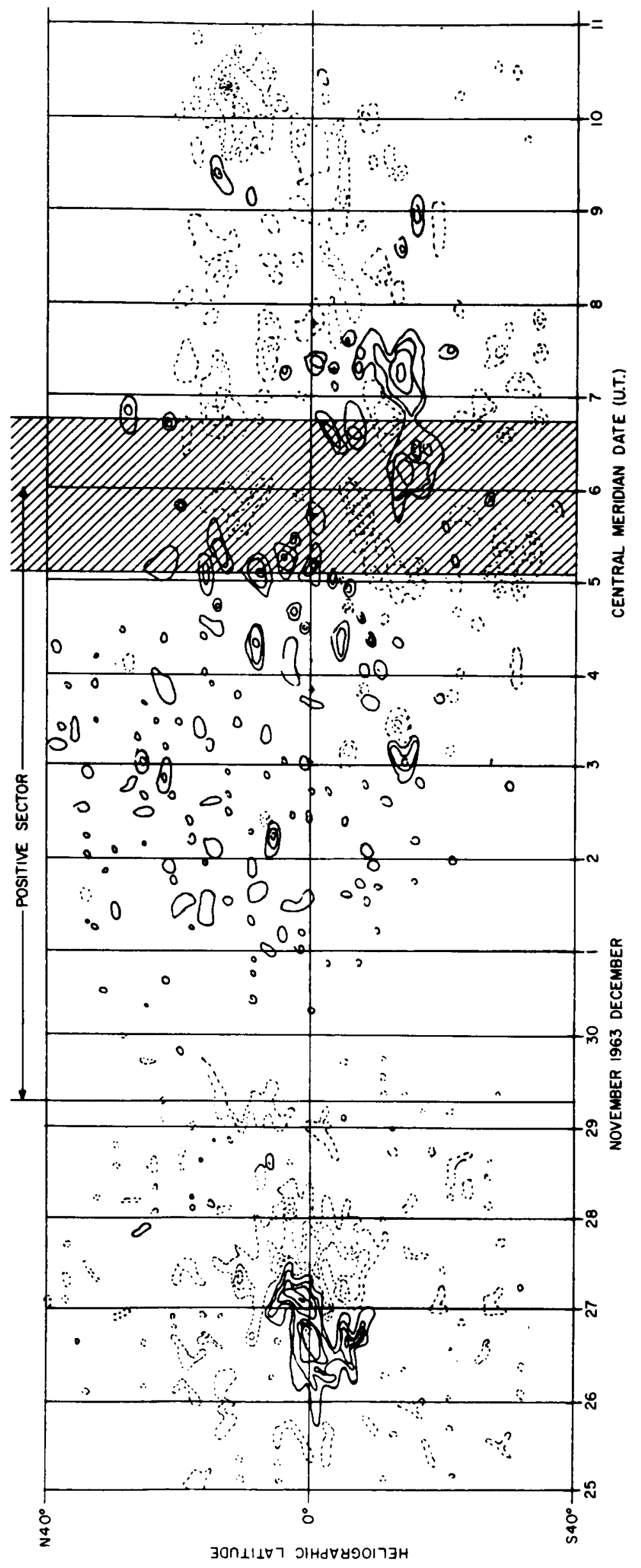

Figure 48 

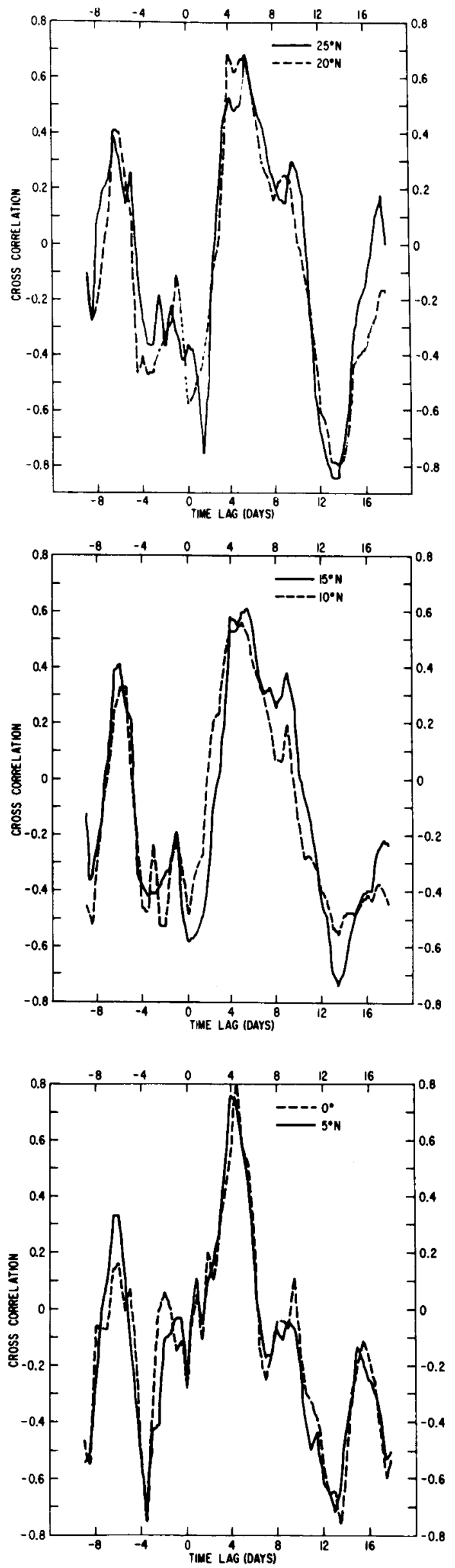

Figure 49 


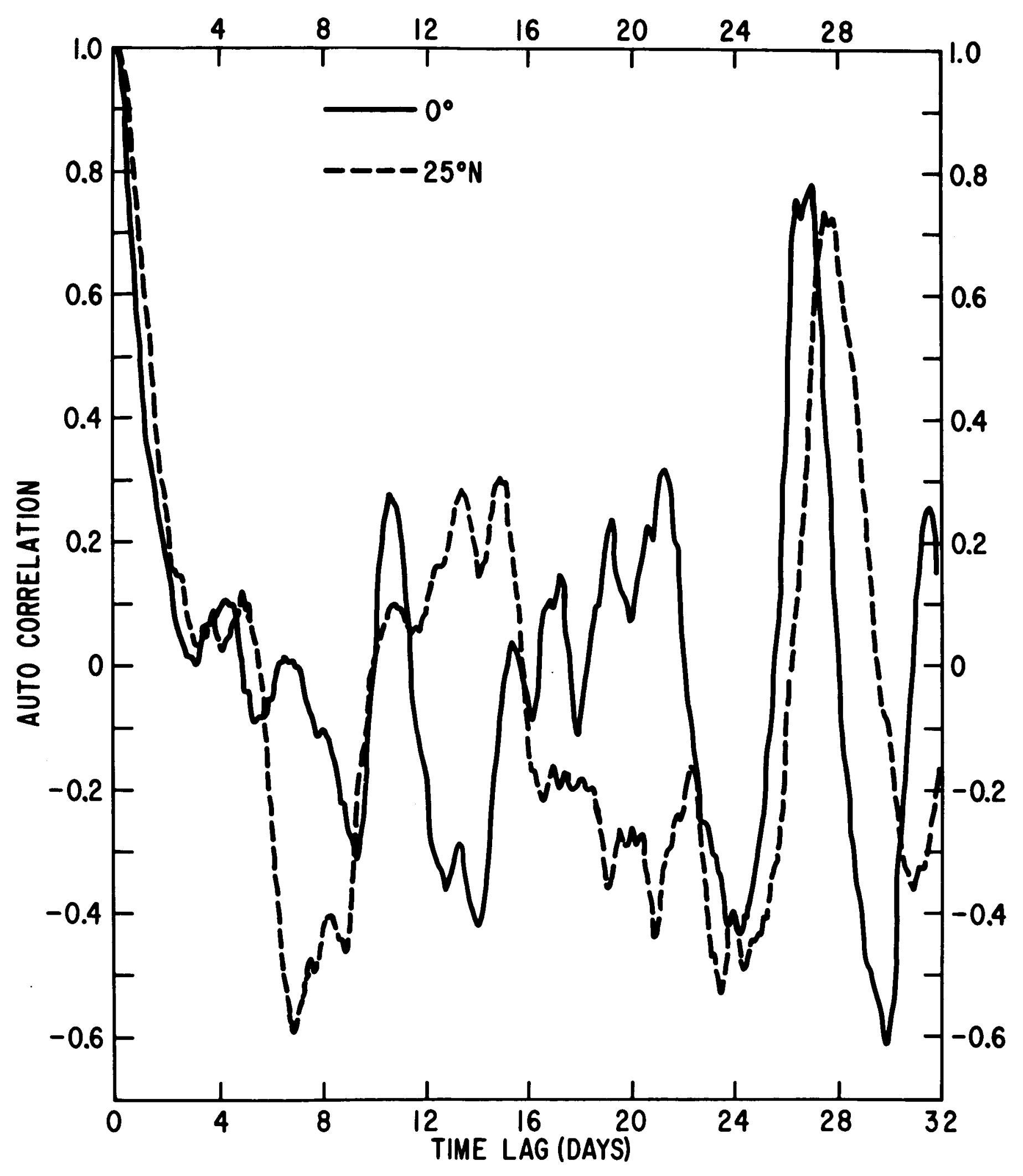




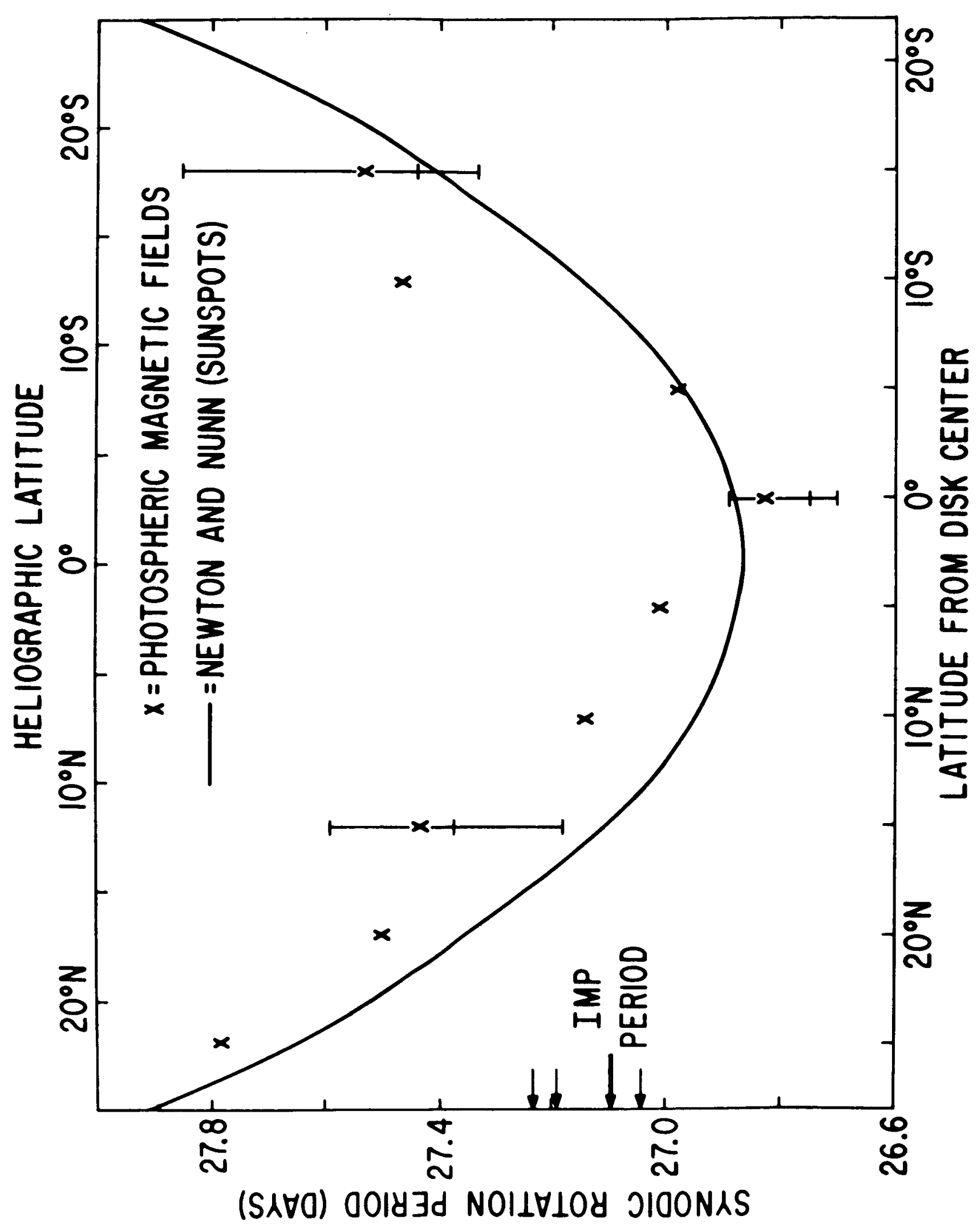

Figure 51 


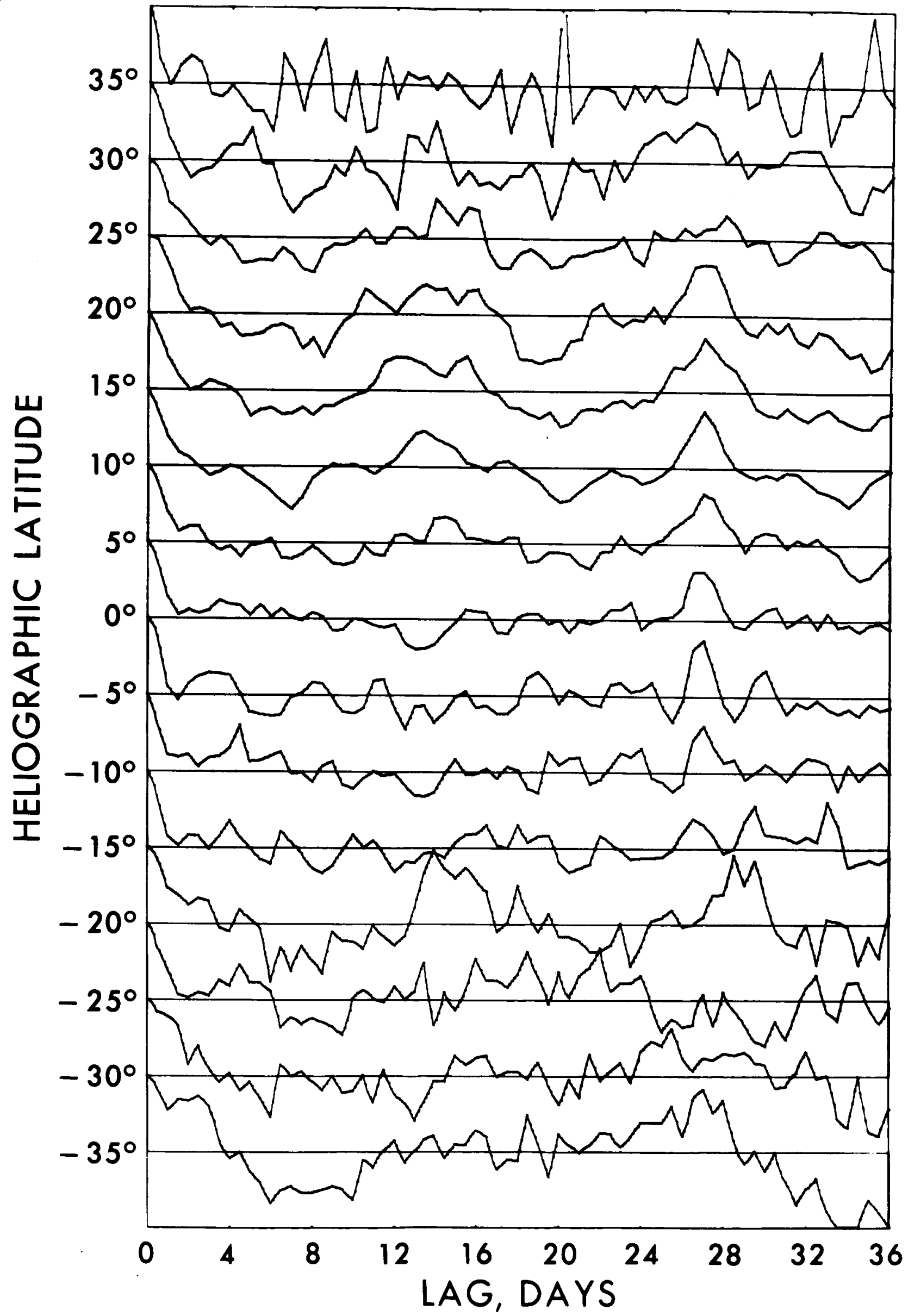

Figure 52 


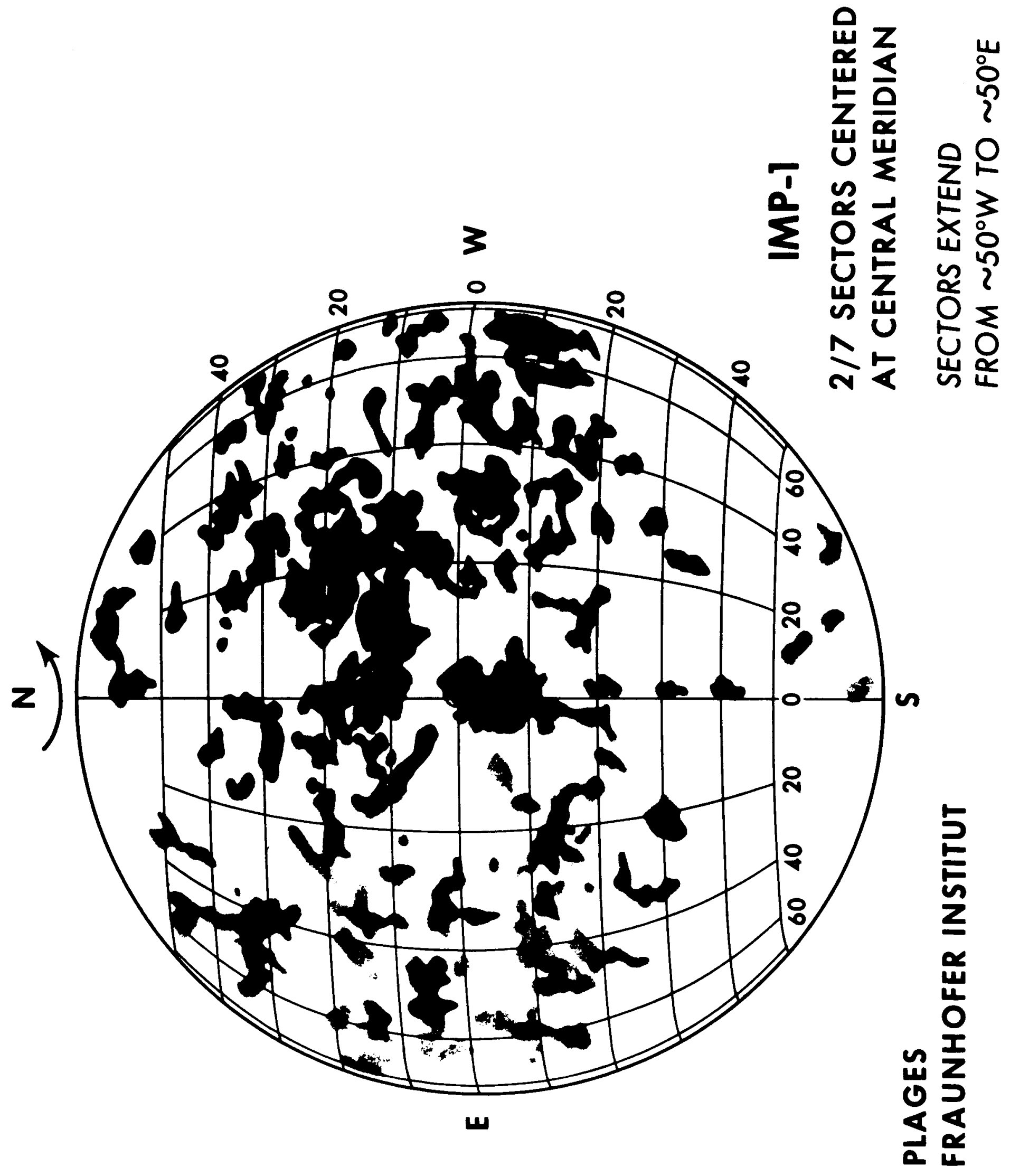




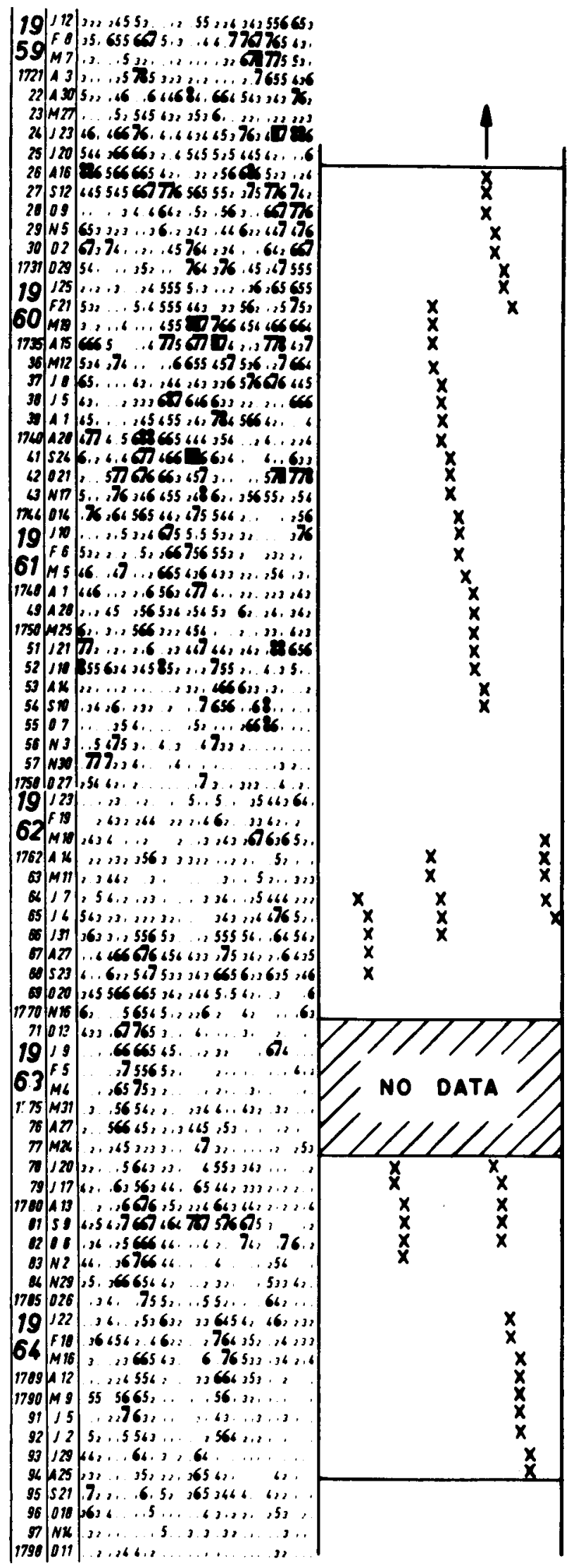

Figure 54 


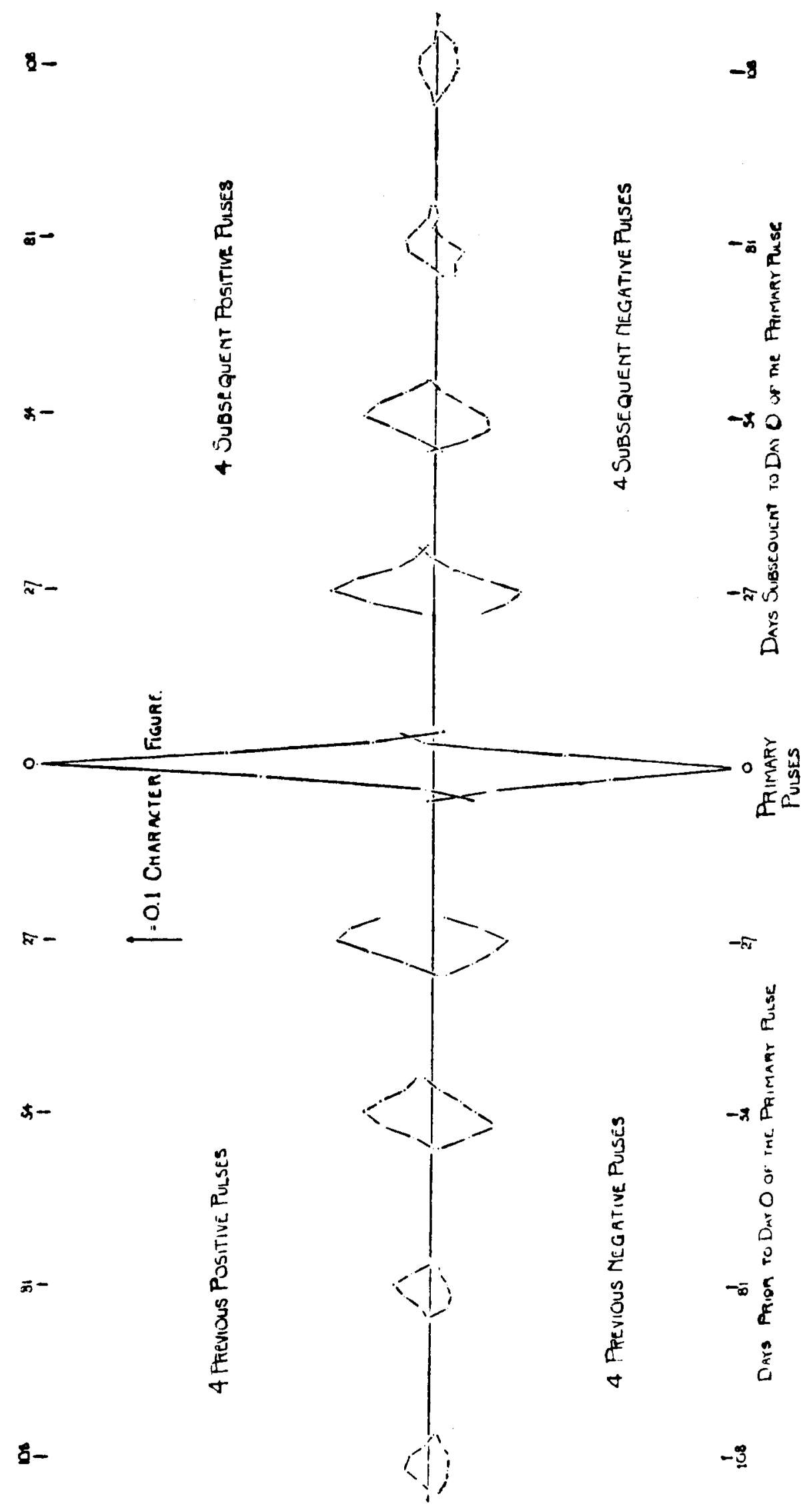

Figure 55 


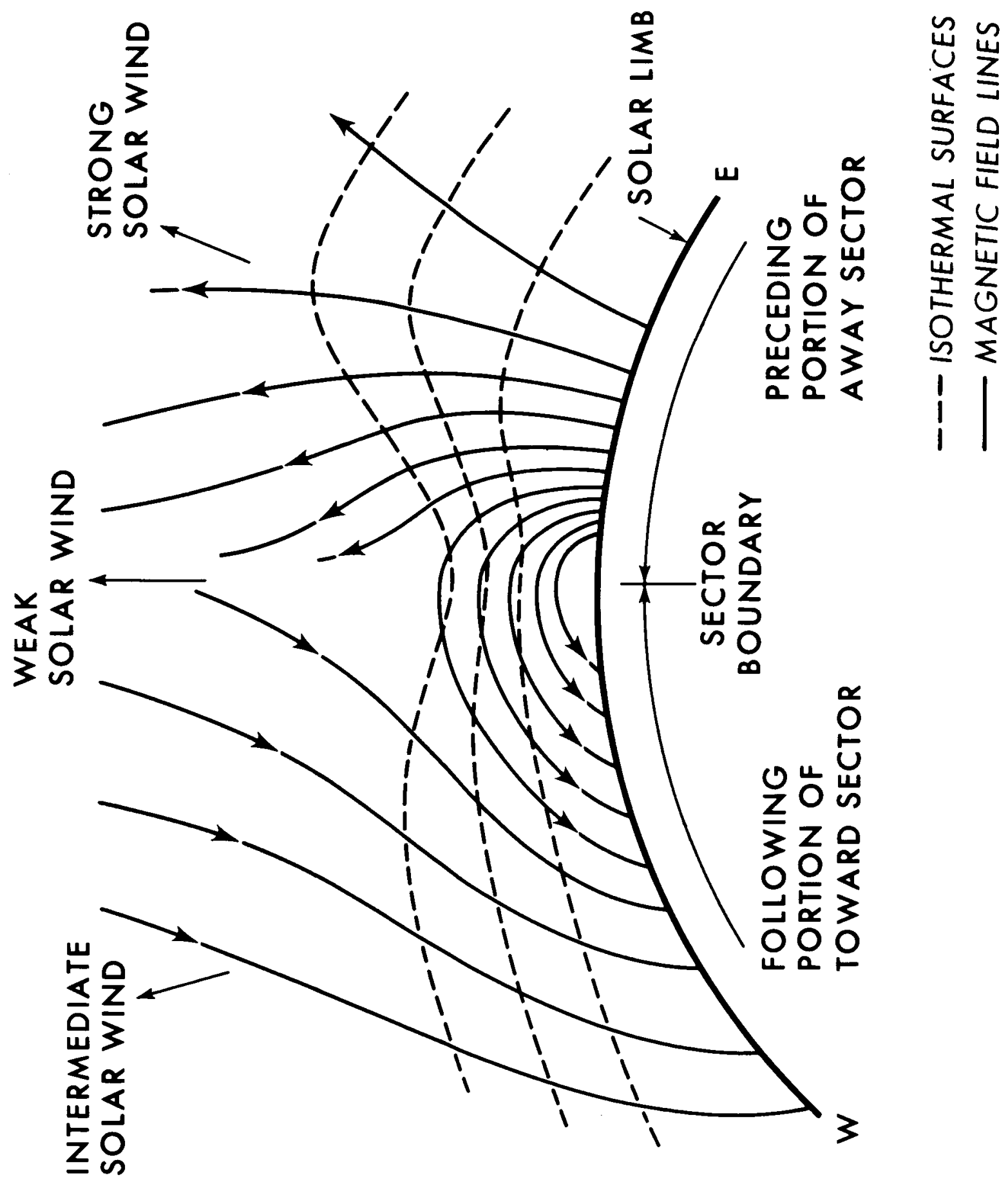




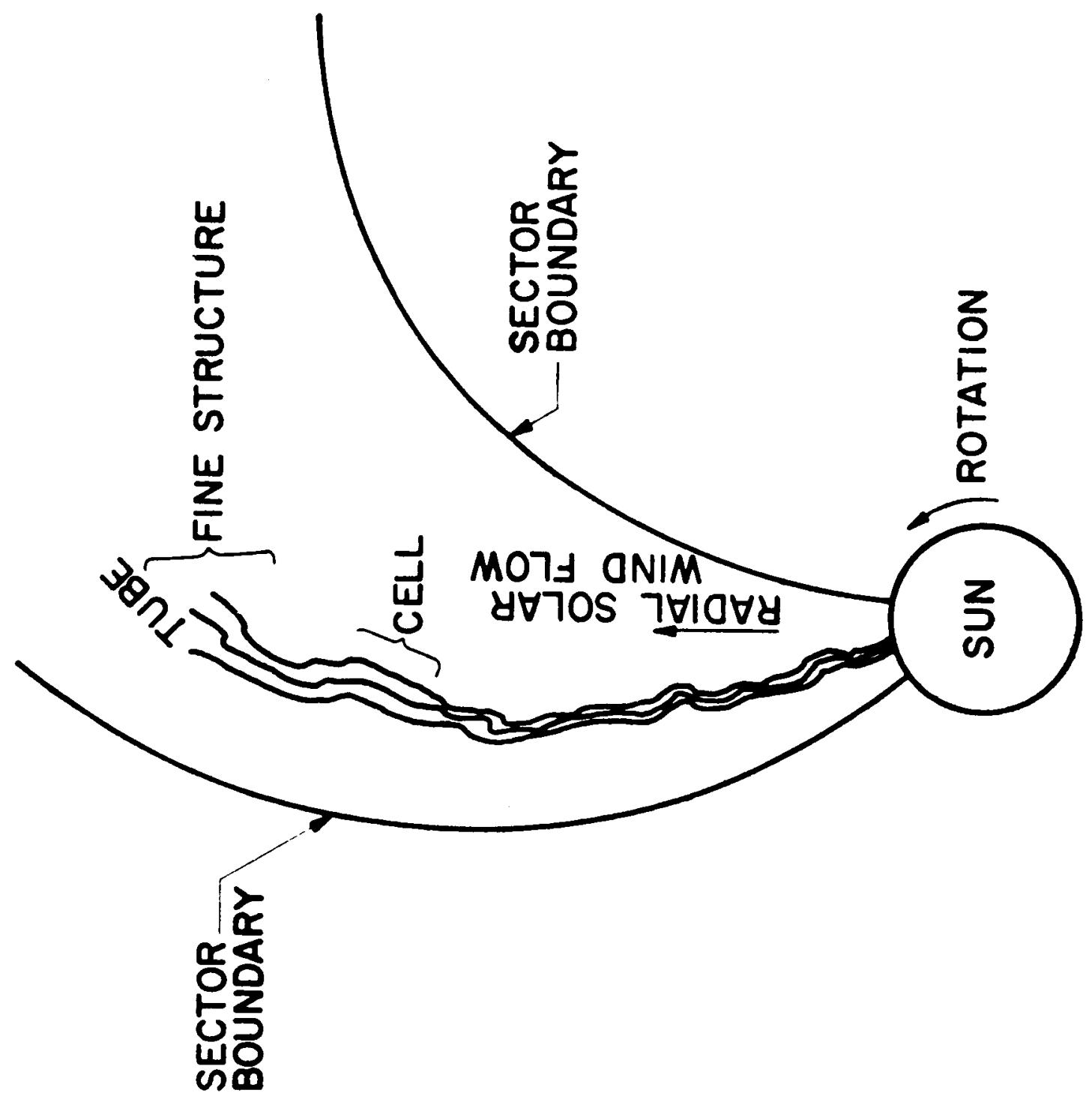




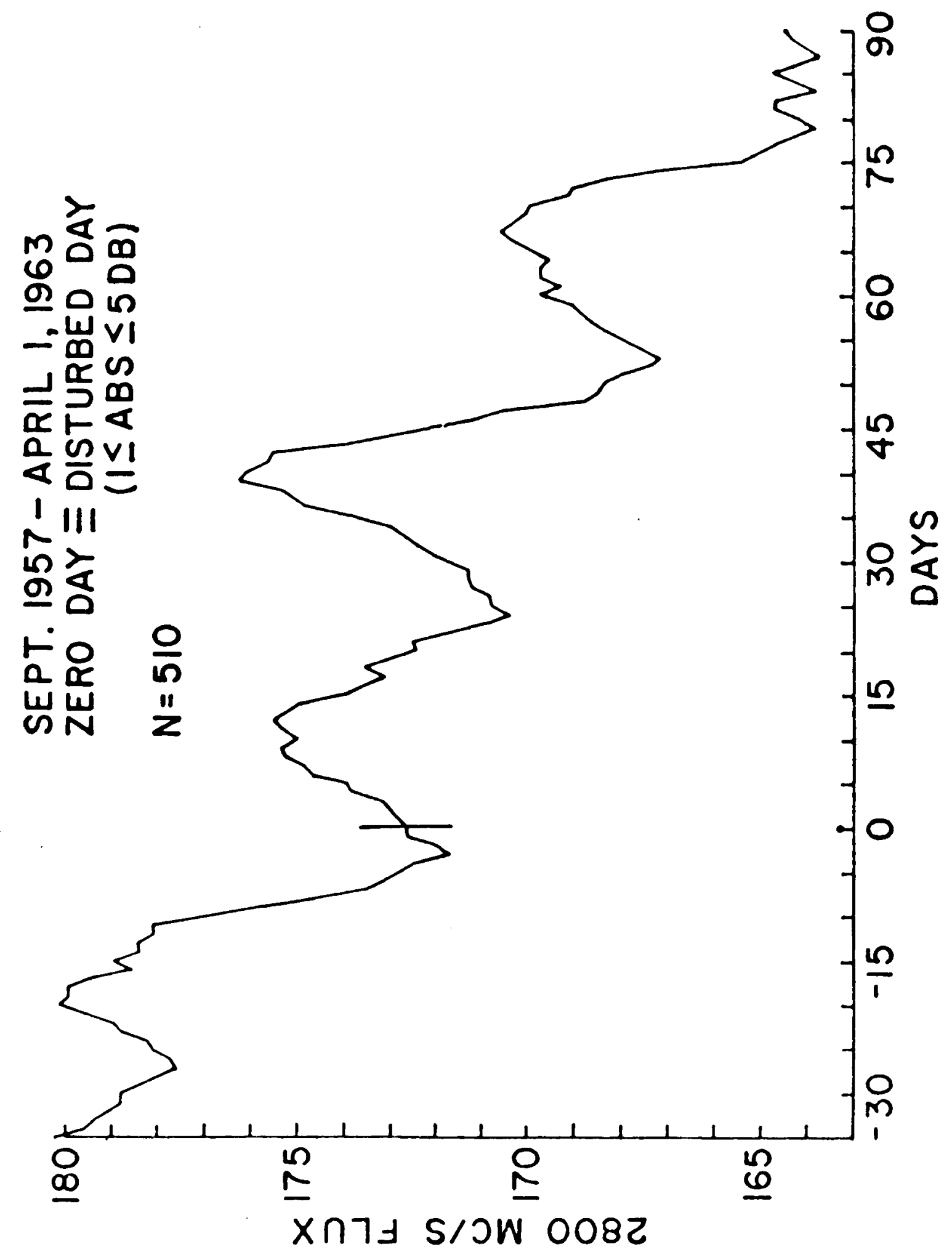

Figure 58 


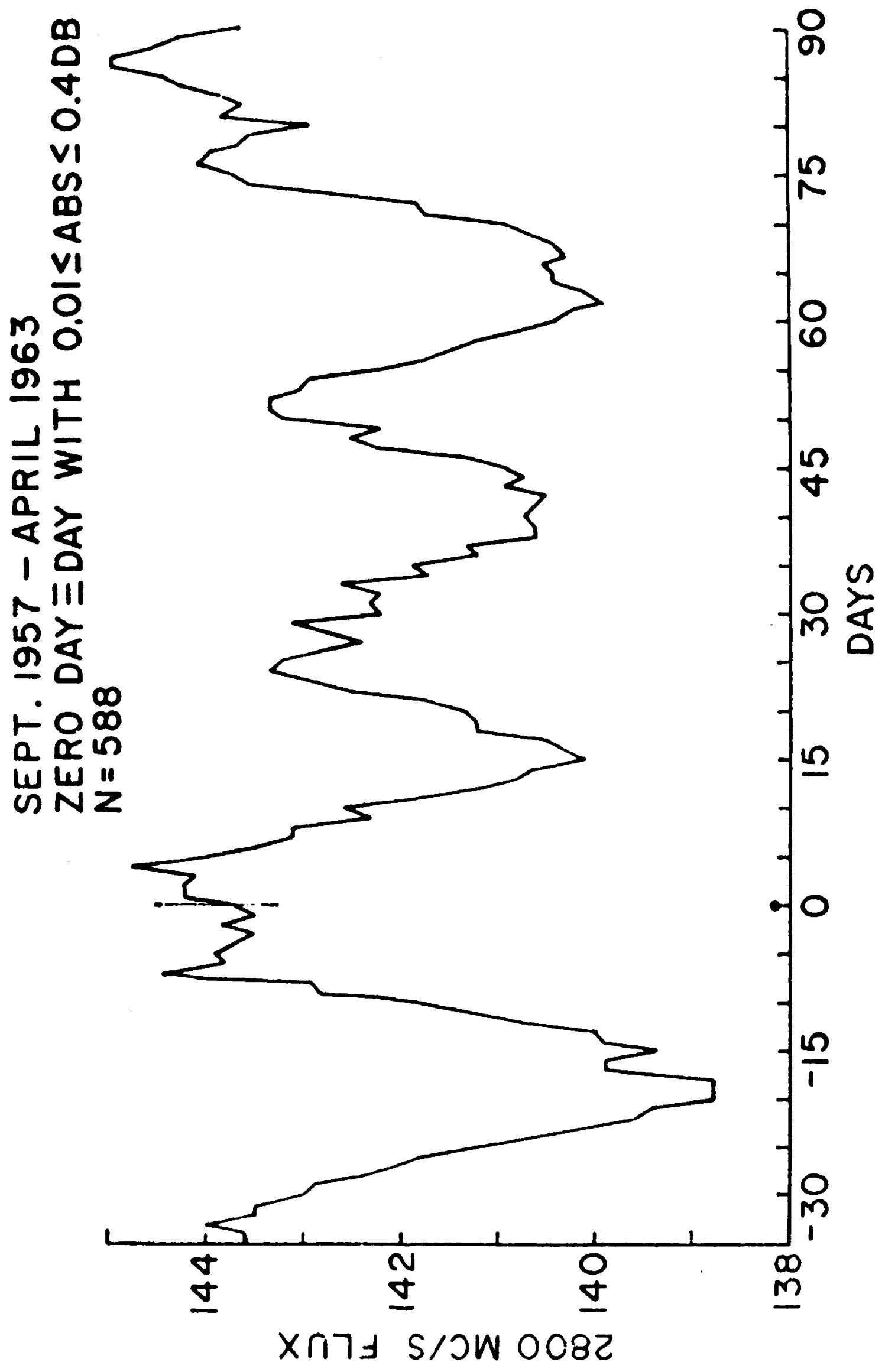

Figure 59 


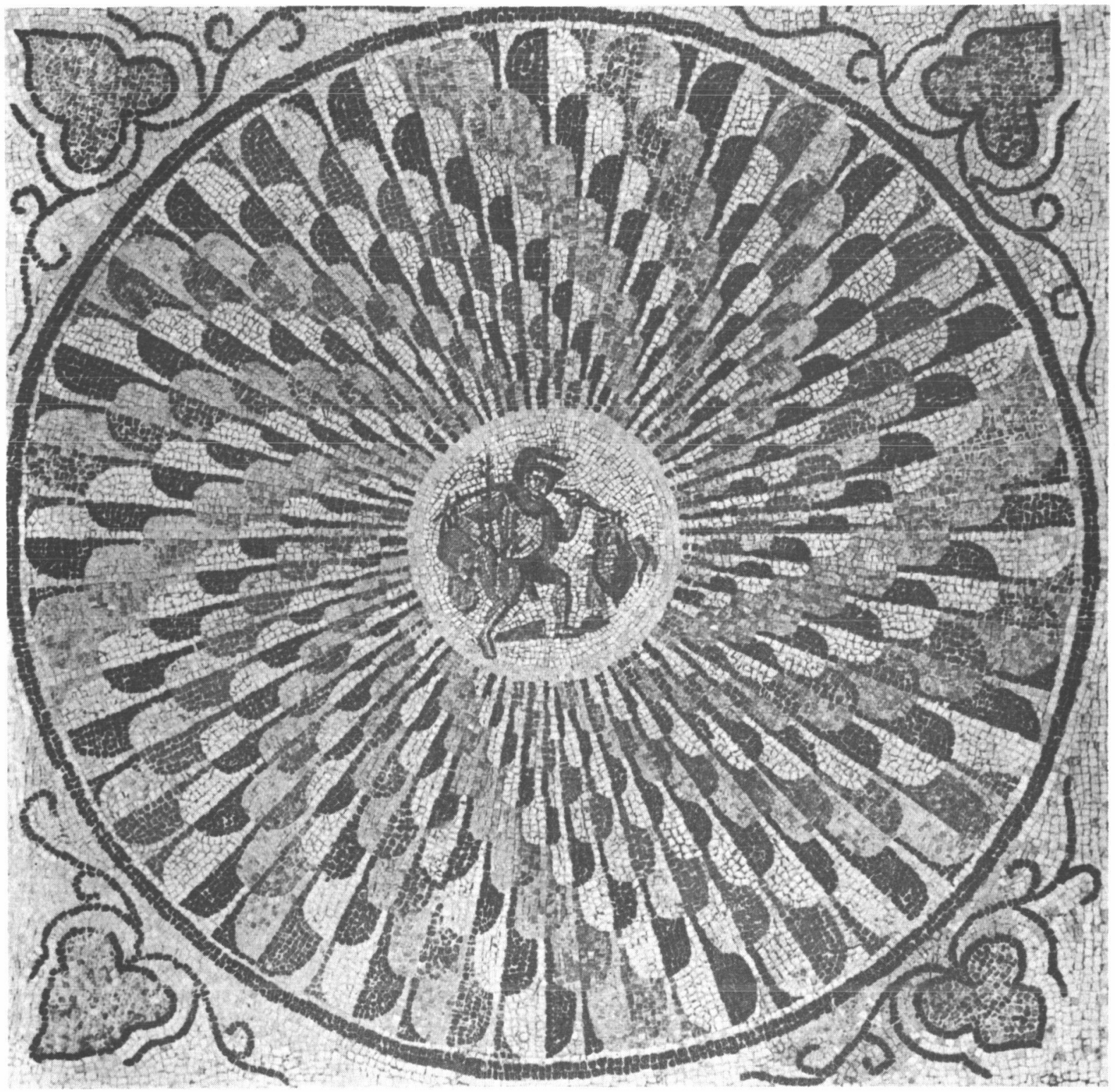


DOCUMENT CONTROL DATA - R\&D

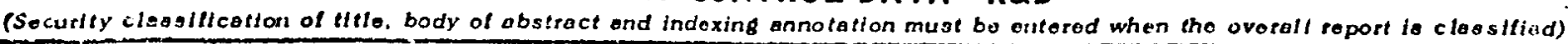

\begin{tabular}{|c|c|}
\hline \multirow{2}{*}{$\begin{array}{l}\text { 1. ORIGINATING ACTIVITY (Comorats author) } \\
\text { Space Sciences Laboratory } \\
\text { University of California } \\
\text { Berkeley, California } 94720\end{array}$} & $\begin{array}{l}\text { 2a. REPORT SECURITY CLASSIFICATION } \\
\text { UnClassified }\end{array}$ \\
\hline & 26. GROUP \\
\hline
\end{tabular}

3. REPORT TITLE

THE INTERPLANETARY MAGNETIC FIELD; SOLAR ORIGIN AND TERRESTRIAL EFFECTS

\section{DESCRIPTIVE NOTES (TyPo of report and inclusive dates) \\ Technical Report}

5. AUTHOR(S) (Last name, first name, initial)

Wilcox, John M.

\begin{tabular}{|c|c|c|}
\hline $\begin{array}{l}\text { 6. REPO RT DATE } \\
\text { January 8, } 1968\end{array}$ & $\begin{array}{c}\text { 7a. TOTAL NO. OF PAGES } \\
138\end{array}$ & $\begin{array}{c}\text { 7b. NO. OF REFS } \\
91\end{array}$ \\
\hline $\begin{array}{l}\text { a. CONTRACT OR GRANT NO. } \\
\text { ONR COntract NOnT } 3656(26) \\
\text { b. PROFASA Grants NsG } 243 \text { \& NGR 05-003- }\end{array}$ & $\begin{array}{l}\text { 9. ORIGINATOR'S REPORT } \\
\text { Series NO. 9, }\end{array}$ & $\begin{array}{l}\text { BER(S) } \\
\text { sue No. } 2\end{array}$ \\
\hline c. & 96. OTHERRERORT NO(S) & other numbers that may bo assigned \\
\hline
\end{tabular}

10. AVAILABILITY/LIMITATION NOTICES

Qualified requesters may obtain copies of this report from DDC.

11. SUPPL EMENTARY NOTES

12. SPONSORING MILITARY ACTIVITY

Nuclear Physics Branch

Office of Naval Research

Washington, D. C. 20360

13. ABSTRACT Many observations related to the large-scale structure of the interplanetary magnetic field, its solar origin and terrestrial effects are discussed. Durin the period observed by spacecraft the interplanetary field was dominated by a sector structure corotating with the sun in which the field is predominantly away from the sun (on the average in the Archimedes spiral direction) for several days (as observed near the earth), and then toward the sun for several days, etc. The average sector appears to be a coherent entity with internal structure such that its preceding portion is more active than its following portion. Cosmic rays corotate with the interplanetary field, and there are differential flows associated with the sector pattern. Profound effects on geomagnetic activity and the radiation belts are produced as the sector pattern rotates past the earth. The solar origin of the sector pattern is discussed. The solar source may be associated 